\title{
UTILIZING PERMANENT ON-BOARD WATER STORAGE FOR EFFICIENT DEEP SPACE RADIATION SHIELDING
}

\author{
A Thesis \\ presented to \\ the Faculty of California Polytechnic State University, \\ San Luis Obispo
}

\author{
In Partial Fulfillment \\ of the Requirements for the Degree \\ Master of Science in Aerospace Engineering
}

by

Nathan Gehrke

June 2018 
(C) 2018

Nathan Gehrke

ALL RIGHTS RESERVED 


\section{COMMITTEE MEMBERSHIP}

TITLE:

AUTHOR:

DATE SUBMITTED: June 2018

COMMITTEE CHAIR: Kira Abercromby, Ph.D.

Associated Professor of Aerospace Engineering

COMMITTEE MEMBER: Amelia Greig, Ph.D.

Assistant Professor of Aerospace Engineering

COMMITTEE MEMBER: Eric Mehiel, Ph.D.

Professor of Aerospace Engineering

COMMitTeE MEMBER: Jennifer Klay, Ph.D.

Associate Professor of Physics 


\begin{abstract}
Utilizing Permanent On-Board Water Storage for Efficient Deep Space Radiation Shielding

Nathan Gehrke
\end{abstract}

As space technologies continue to develop rapidly, there is a common desire to launch astronauts beyond the ISS to return to the Moon and put human footsteps on Mars. One of the largest hurdles that still needs to be addressed is the protection of astronauts from the radiation environment seen in deep space. The most effective way to defend against radiation is increasing the thickness of the shield, however this is limited by strict mass requirements. In order to increase the thickness of the shield, it is beneficial to make mission critical items double as shielding material.

The human rated Orion spacecraft has procedures in place for astronauts to create an emergency bunker using food and water in the event of a forewarned radiation storm. This can provide substantial support to defend against radiation storms when there is an adequate amount of warning time, however, fails to protect against Galactic Cosmic Radiation (GCR) or Solar Particle Events (SPE) without sufficient warning. Utilizing these materials as a permanent shielding method throughout the mission could be a beneficial alternative to the Orion programs current protection plan to provide constant safety to the crew.

This thesis analyzes the effect in the radiation dosage seen by astronauts in the Orion Crew Module through use of on-board water as a permanent shielding fixture. The primary method used to analyze radiation is NASA's OLTARIS (On-Line Tool for the Assessment of Radiation In Space) program, which enables users to input thickness distributions to determine a mission dosage profile. In addition this thesis further develops a ray tracing code which enables users to import male and female models into the vehicle model to produce gender specific radiation dosage results. 
The data suggests the permanent inclusion of water as a shielding material provides added support for GCR as well as SPE radiation that can extend the mission lifetime of humans in space. 


\section{ACKNOWLEDGMENTS}

Thanks to:

- Dr. A for her continuous support throughout the scope of the project, as well as being an inspiration for future life endeavors.

- Chris Sandridge for his support and critical debugging with OLTARIS through the thick and thin of radiation testing for this project.

- My parents for constant love and support throughout my time in the undergraduate and graduate school.

- The Gehrke and Crain families including: Jack and Barbara Crain, and Dave and Anitra Gehrke for the continuous love and inspiration that are absolutely unmatched.

- Chuck and Twyla Martin for being the unwavering educational backbone of the phenomenal Gehrke and Janc families.

- To all mentioned, and all that should have been mentioned - I love you! 


\section{TABLE OF CONTENTS}

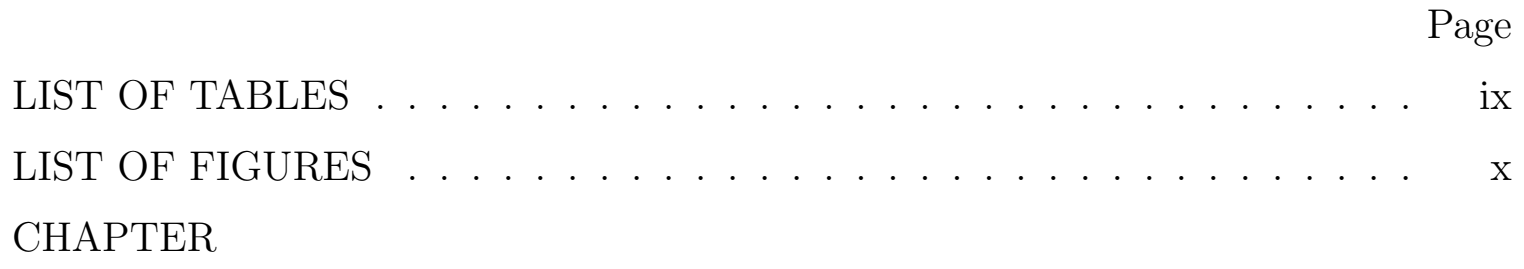

1 Background ............................... 1

1.1 Overview .............................. 1

1.2 Project Relevance . . . . . . . . . . . . . . . . . . 2

2 General Background . . . . . . . . . . . . . . . 5

2.1 Radiation Environment . . . . . . . . . . . . 5

2.1.1 Galactic Cosmic Rays (GCR) . . . . . . . . . . 6

2.1.2 Secondary Cosmic Rays . . . . . . . . . . . . . . 7

2.1.3 Solar Radiation . . . . . . . . . . . . . . . 8

2.1.4 Solar Particle Events (SPE) . . . . . . . . . . . . . 10

2.2 Spacecraft in Radiation Environment . . . . . . . . . . . . . 13

2.2.1 Active Shielding . . . . . . . . . . . . . 13

2.2.2 Passive Shielding . . . . . . . . . . . . . . . 14

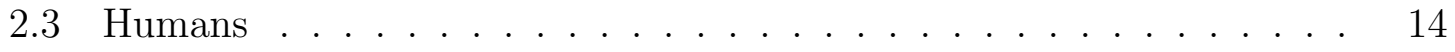

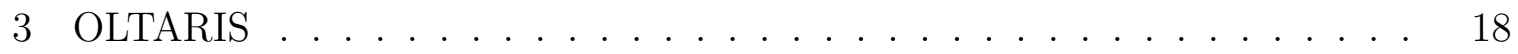

3.1 General Background ................... 18

3.2 OLTARIS Calculation Process . . . . . . . . . . . . . . 19

3.2.1 HZETRN2005 ......................... 20

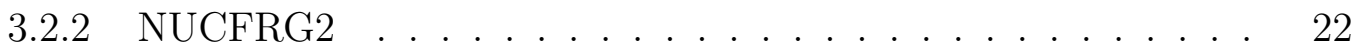

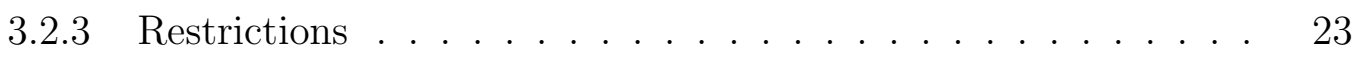

3.3 Thickness Distributions . . . . . . . . . . . . . 23

3.3.1 Phantom Bodies . . . . . . . . . . . . . 25

3.4 Material Properties . . . . . . . . . . . . . . 27

3.5 Radiation Environment . . . . . . . . . . . . . . 27

3.5.1 Historical SPE . . . . . . . . . . . . . . . . 29

3.6 Response Functions . . . . . . . . . . . . . . . . . . . . . 32 
3.6 .1 Dose . . . . . . . . . . . . . . . . . . . . . . 32

3.6 .2 Dose Equivalent . . . . . . . . . . . . . . . . . . . . . . 33

3.6 .3 Effective Dose Equivalent . . . . . . . . . . . . . . 34

3.6.4 Linear Energy Transfer (LET) . . . . . . . . . . . . . . 36

3.7 Verification . . . . . . . . . . . . . . . . . . . 36

4 ORION Crew Module . . . . . . . . . . . . . . . . . . 38

4.0 .1 Limitations . . . . . . . . . . . . . . . . . . . 40

4.1 Dimensions and Capabilities . . . . . . . . . . . . . . . 40

4.1.1 Emergency Radiation Protection Plan . . . . . . . . . . . . 41

5 OLTARIS Tool . . . . . . . . . . . . . . . . . . . . . 44

5.1 Tool Functionality . . . . . . . . . . . . . . . . . . 44

5.2 Revisions . . . . . . . . . . . . . . . . . . 48

5.3 Limitations . . . . . . . . . . . . . . . . . . . . . . . . . 54

5.4 Orion Crew Module Simplifications . . . . . . . . . . . . 55

6 Radiation Testing Setup . . . . . . . . . . . . . . . . . . . 58

6.1 SPE Environment . . . . . . . . . . . . . . . . . . . 60

6.2 GCR Environment . . . . . . . . . . . . . . . . . . . . 61

6.3 Uncertainty . . . . . . . . . . . . . . . . . . 62

7 Results . . . . . . . . . . . . . . . . . . . . . 64

7.1 SPE Analysis . . . . . . . . . . . . . . . . . . . . . . 64

7.1.1 Effect of Water . . . . . . . . . . . . . . . 65

7.1 .2 Carrington Event . . . . . . . . . . . . . 66

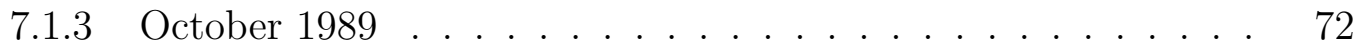

7.2 GCR Analysis . . . . . . . . . . . . . . . . . . 75

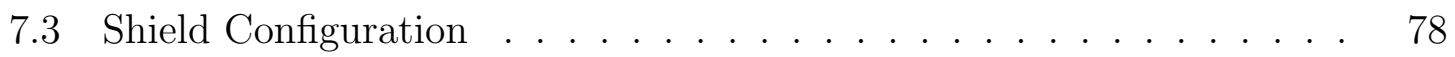

7.4 Shield Thickness . . . . . . . . . . . . . . . . . . . . . 79

8 Conclusion . . . . . . . . . . . . . . . . . . . . . . 81

8.1 Future Work . . . . . . . . . . . . . . . . . . . . . . . 82

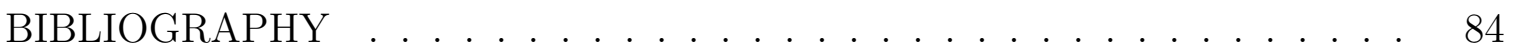




\section{LIST OF TABLES}

Table

Page

5.1 Ray Distribution Associated Variance . . . . . . . . . . . . . . 48

5.2 New Material Implementation . . . . . . . . . . . . . . . 52

6.1 Solar Particle Event Comparisons . . . . . . . . . . . . . . 61

6.2 Galactic Cosmic Ray Comparisons . . . . . . . . . . . . . . 61

7.1 Carrington Event Radiation Exposure: Long-Term Effects . . . . . 65

7.2 October 1989 Event Radiation Exposure: Long-Term Effects . . . . 65

7.3 Dose Equivalent Comparison Including Bladder . . . . . . . . . 66

7.4 Carrington Event Radiation Exposure: Long-Term Effects . . . . . 67

7.5 Carrington Event Radiation Exposure: Short-Term Effects . . . . . 67

7.6 Radiation Exposure with Water on Exterior: Long-Term Effects . 68

7.7 Radiation Exposure with Water on Exterior: Short-Term Effects . . 69

7.8 Results of Increasing Shield Thickness . . . . . . . . . . . . . 72

7.9 October 1989 Event Radiation Exposure: Long-Term Effects . . . . 72

7.10 October 1989 Event Radiation Exposure: Short-Term Effects . . . . 73

7.11 Radiation Exposure with Water on Exterior: Long-Term Effects . . 73

7.12 Radiation Exposure with Water on Exterior: Short-Term Effects . . 73

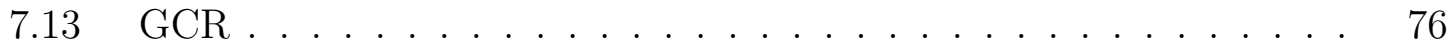




\section{LIST OF FIGURES}

Figure $\quad$ Page

1.1 General effects of water as a radiation shield $[27] \ldots$. . . . . . . . 3

2.1 Major Solar Particle Events[3] . . . . . . . . . . . . . 6

2.2 GCR particle breakdown beyond a $3 \mathrm{~g} / \mathrm{cm}^{2}$ Aluminum shield [24] $\quad 7$

2.3 Abundance of elements in the GCR environment (1977)[19] . . . . . 8

2.4 GCR flux at solar minima and maxima[19] . . . . . . . . . . . . . 9

2.5 Major Solar Particle Events[3] . . . . . . . . . . . . . 11

2.6 Solar magnetic fields $[21] \ldots \ldots$. . . . . . . . . . . . . . 11

2.7 Solar Particle Events $[24]$. . . . . . . . . . . . . . . . . 12

2.8 NASA Permissible Exposure Limits[25] . . . . . . . . . . . 15

2.9 NASA Radiation Limits[22] . . . . . . . . . . . . . 16

2.10 NASA Radiation Limit Comparison $[5] \ldots \ldots$. . . . . . . . . 16

2.11 NASA Radiation Penetration and Exposure Limits[22] . . . . . . . 17

3.1 OLTARIS Program Flow $[28] \ldots \ldots$. . . . . . . . . 20

3.2 Example Thickness Distribution . . . . . . . . . . . . . . . . 24

3.3 Ray Distributions Accessable Through OLTARIS[10] . . . . . . 25

3.4 Phantom Bodies available for download through OLTARIS[28] . . . 26

3.5 OLTARIS GCR Model Comparison[29] . . . . . . . . . . . . . . 28

3.6 Carrington SPE Model[17] . . . . . . . . . . . . . . 31

3.7 Example Effective Dose in OLTARIS . . . . . . . . . . . . 35

3.8 Tissue Weighting Factors[28] . . . . . . . . . . . . . . . . 35

4.1 Orion Spacecraft $[18] \ldots \ldots$. . . . . . . . . . . . . . 38

4.2 Orion Crew Module[13] . . . . . . . . . . . . . . . . 39

4.3 Emergency Radiation Protection Plan[7] . . . . . . . . . . . . 42

4.4 Emergency Radiation Protection Plan[14] . . . . . . . . . . 43

5.1 CAD model of sphere imported to MATLAB . . . . . . . . . 45

5.2 Intersection Point Algorithm[1] . . . . . . . . . . . . 46 
5.3 MATLAB Vehicle Thickness Distribution Tool Flow Chart[10] . . . 47

$5.4 \quad$ Undefined Ray Thickness Process . . . . . . . . . . . . . . 50

$5.5 \quad$ Multiple Undefined Rays Process . . . . . . . . . . . . . . 50

5.6 Visual of $25 \%$ of Rays Covered in New Material . . . . . . . . 53

5.7 Orion CAD processed by MATLAB tool . . . . . . . . . 54

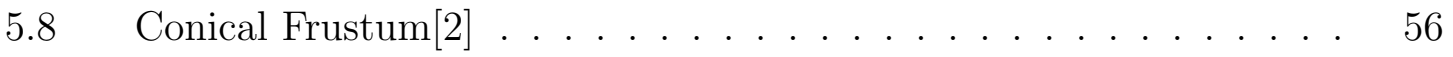

5.9 CAD model of simplified Orion sphere. . . . . . . . . . . 57

7.1 Orion Shield Cutout with Water Configuration _. . . . . . 68

7.2 Water Shield Configuration within the SPE Environment . . . . . 69

7.3 Radiation Dosage Vs Shielding Thickness for Carrington Event . . 71

7.4 Radiation Dosage Vs Shielding Thickness for the October 1989 Event 75

7.5 Water Shield Configuration within GCR environemnt . . . . . 77

7.6 Radiation Dosage Vs Shielding Thickness in the GCR Environment 78 


\section{Chapter 1}

\section{BACKGROUND}

Space technologies have been developing rapidly over the last several decades, opening the window for manned missions beyond the International Space Station (ISS). It has become a common desire among governments and private companies alike to bring men and women on missions beyond the ISS to the Moon, Mars, and beyond.

One of the largest hurdles that still needs to be addressed is the protection of astronauts from the radiation environment of deep space. The environment beyond the Van Allen belts is far less protected than what humans have faced in Low Earth Orbit (LEO), which leads to specific engineering challenges. The best option to protect against radiation is increasing the thickness of shielding material, however, there is a limit as the thickness of shielding directly affects the mass of the spacecraft.

In order to embark on a manned mission into deep space, it is critical to prove the astronauts will be protected and held under the maximum mission radiation dosage. There are several proposed options by NASA and other companies to counteract the harsh radiation environment that are in development today. This paper will look to improve upon these designs by studying the effects of enclosing the crew module with the already required on-board water as additional shielding.

\subsection{Overview}

The deep space radiation environment is a dangerous place, and requires serious attention to ensure the safety of astronauts. The United States plans to launch astronauts into space beyond the protection of Earth's magnetosphere as early as 2022 in the Orion capsule, for a return mission to the moon. This thesis proposes that water 
should be incorporated as a full time shield to best protect the astronauts in transit. Current long-duration missions in the planning phase do not utilize water permanently for radiation shielding. Rather, current plans call for emergency situations in which water is used as a bunker to protect astronauts. This paper will investigate the radiation dosages of astronauts aboard the Orion Crew Module for a general deepspace mission. The primary tool for investigation for radiation exposure is OLTARIS, which is accessible through NASA. The water utilized in the shield will be the required amount necessary for humans in the duration of the mission. This will dictate the thickness of the shielding to determine whether it will make a better impact as a full shield as proposed, or temporary shield as it is currently planned.

General background will be discussed in chapter 2 on the radiation environment, shielding techniques, and humans in the space environment. From there, the tool used for testing will be introduced and described. The radiation analysis tool, OLTARIS, will be detailed to provide its capabilities in chapter 3. This program is the main source of testing for this paper to trade between all of the potential shielding schemes. There is additional code required that will also be detailed and verified. Background will then be given on the ORION crew module in chapter 4, which was chosen as the prime subject of the thesis due to its level of development and public access. The crew module will be the baseline and modified for further testing. The testing procedure and steps will then be detailed to show the methodology of the calculations. The OLTARIS results will then be analyzed to determine the best allocation of on-board water in order to mitigate the effects of the radiation environment.

\subsection{Project Relevance}

There is a considerable amount of documentation that indicates hydrogen rich materials are beneficial to radiation shielding. This includes plastics, liquid hydrogen, and 
water. Water is often suggested as a radiation shield option due to its necessity on crewed missions. There have been several papers that document the difference in effectiveness of different materials including water, aluminum, liquid hydrogen, lithium hydride, lead, lunar, and martian regolith[27][6][30]. A NASA Technical Paper[27] in 1991 showed the benefits of utilizing water as radiation shielding, but did not go into detail of the effects the dosages would have on humans. Figure 1.1 shows results from the study, finding that an increased thickness in water can cut dosage levels by over $50 \%$.

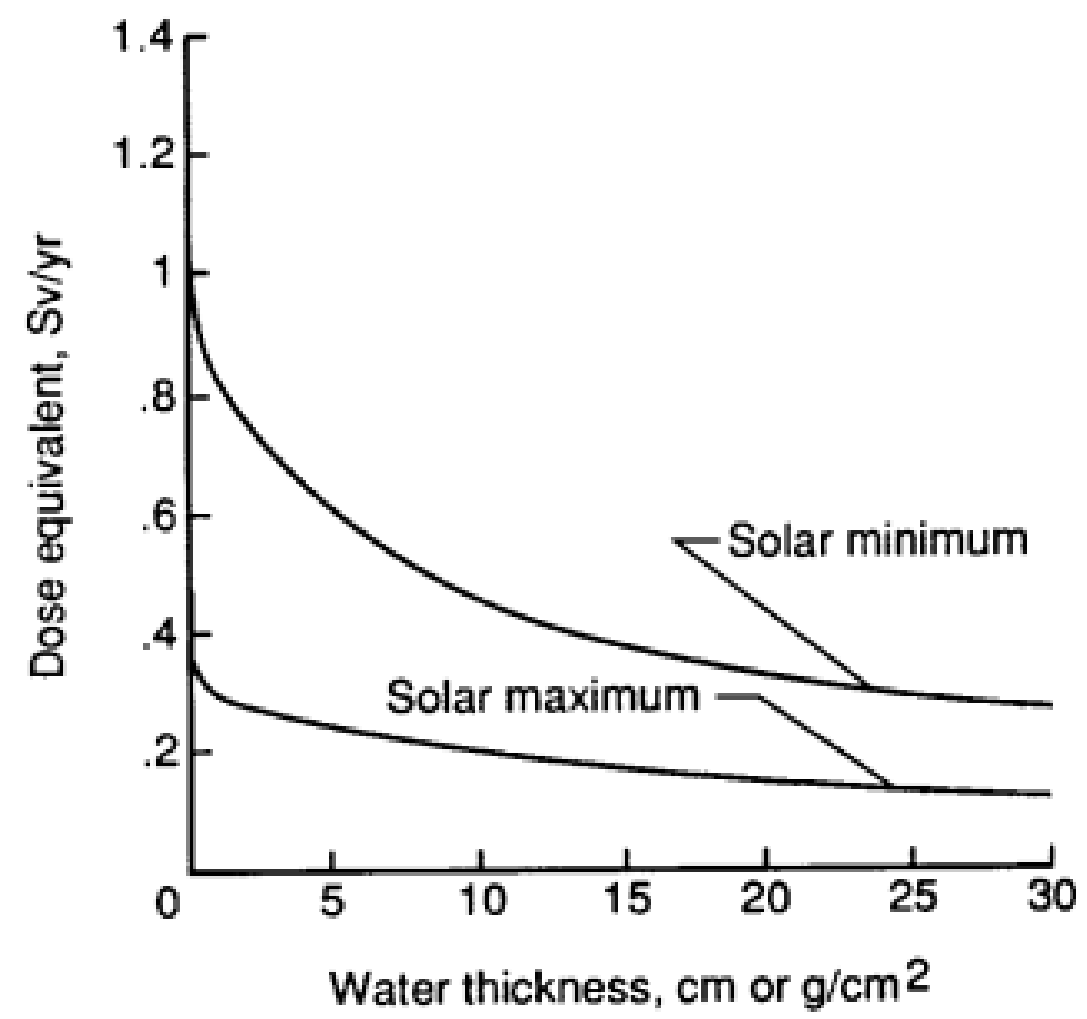

Figure 1.1: General effects of water as a radiation shield[27]

Additional studies have taken place in the following years to show that water, in large enough thicknesses, can reduce the effects of GCR[6]. Even in minimal thicknesses, water has been shown to reduce the effects of radiation shielding[6]. Despite this, there are no major plans to include water as a component of radiation 
shielding. The Orion program from NASA is nearing its first crewed flight beyond the safety of the Van Allen belts, however, it fails to utilize on-board water. Only in the event of a massive solar storm will astronauts prepare an emergency bunker.

This project is intended to continue showing the benefits water has as radiation shielding. In order to advance the discussion, this study will focus exclusively on the Orion capsule to show the benefits of utilizing water as permanent shielding. Water has often been suggested as a possible shielding technique, however it is not currently used as a full time shield. Current designs call for utilizing water as a temporary shield in the event of a warned solar event, but it could be beneficial to investigate the utility of coating the entire spacecraft to bolster up the shielding. Ideally, this study can show that the current NASA design could be improved to help astronauts step foot on Mars. 
Chapter 2

\section{GENERAL BACKGROUND}

The radiation environment is a dynamic, fluctuating environment. Earth has a system of magnetic belts, the Van Allen belts, that protect the planet from many harmful particles. These belts are composed of magnetic particles gathered from the planet's polarity. Outside of the belts, the effects of radiation become much more considerable.

It is difficult to estimate the radiation profile a spacecraft will be exposed to over the duration of its mission. This uncertainty leads to a necessity to over-design spacecraft shielding to ensure mission success, especially for a crewed mission[20].

\subsection{Radiation Environment}

This study is focused on the radiation environment beyond the Van Allen belts for interplanetary missions, specifically for human missions.

There are a variety of types of radiation sources beyond the protection of Earth's magnetic shield. Radiation is emitted from the sun as well as sources outside the solar system. Both possess a unique threat to manned long-duration missions that must be addressed. Figure 2.1 gives a simple breakdown of the radiation environment. This includes the radiation from the sun as well as background radiation from the Milky Way Galaxy. The Van Allen belts are part of the Earth's magnetosphere, which protect the planet from harmful radiation. The Van Allen belts are shown in blue in Figure 2.1. As seen in the image, the belts help deflects harmful rays from the sun. Crewed missions beyond the belts lead to a dangerous radiation environment that

requires astute attention from spacecraft designers. Each of the components of this environment will be detailed in the following sections. 


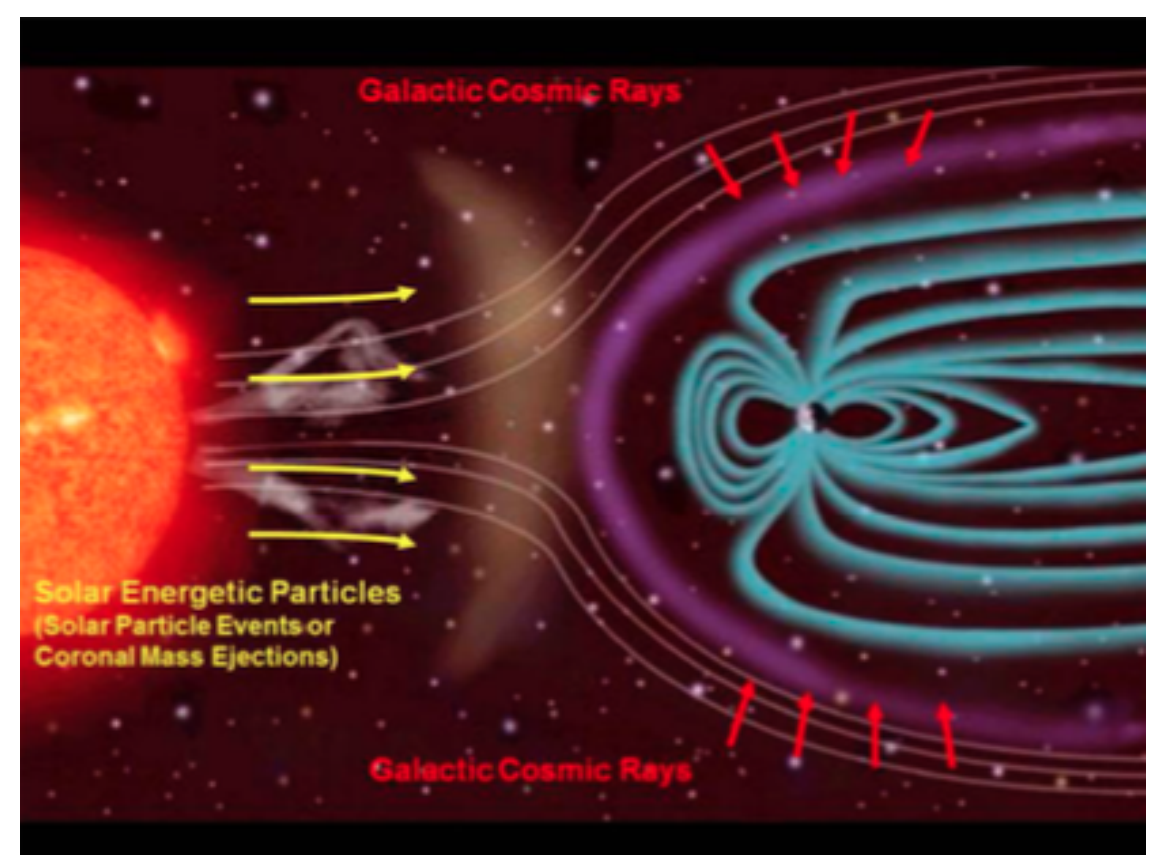

Figure 2.1: Major Solar Particle Events[3]

\subsubsection{Galactic Cosmic Rays (GCR)}

Galactic Cosmic Rays are composed of atomic nuclei from outside the solar system. These particles move at extremely high speeds throughout the galaxy contained by the galactic magnetic field. The nuclei have had all electrons stripped away from the near speed-of-light journey. The effect of the GCR particles is isotropic due to the helical nature of the path the particles take. The GCR radiation environment is difficult to predict and there is only limited data to use in modeling the environment. One trend that has been documented is the correlation between the GCR environment and the solar cycle[19]. At solar maximum, the GCR environment is considerably less than intense than at solar minimum.

GCR consists mostly of simple protons, with only 1-2 percent being composed of High charge (Z) and Energy nuclei (HZE). A majority of the GCR makeup consists of protons from stripped away Hydrogen and Helium atoms. Despite this disparity, the HZE particles present a larger health risk to astronauts than the protons. HZE 
particles have high ionization energy making it impossible to completely shield with modern techniques. Figure 2.2 shows the breakdown by particle of GCRs that penetrate shielding, revealing the threat of HZE particles. The high ionizing power of the particles enable them to penetrate through shielding effectively, creating a large risk for astronauts.

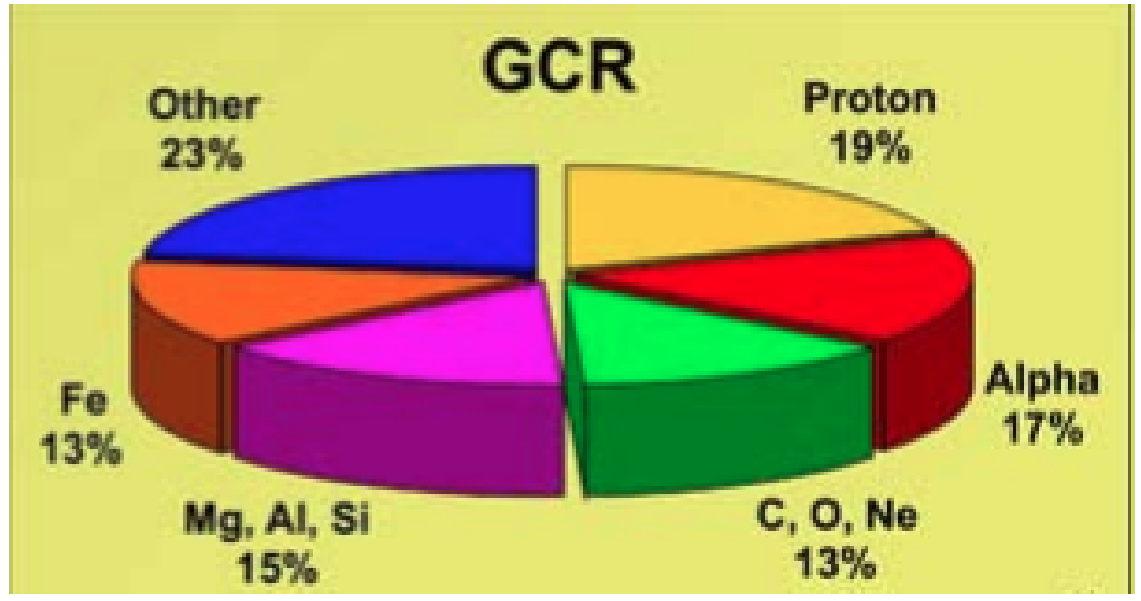

Figure 2.2: GCR particle breakdown beyond a $3 \mathrm{~g} / \mathrm{cm}^{2}$ Aluminum shield $[24]$

Figure 2.3 provides a breakdown of the GCR makeup from a study in the 1977 solar minimum period. As described earlier, a majority of the environment consists of simple protons and Helium nuclei. The broad spectrum of energetic particles present in the GCR environment is a complex field that is difficult to shield against. Figure 2.4 details the energy spectrum of the particles versus the fluence separated by the charge (Z) of the particle[19]. It is apparent from Figure 2.4 that the flux of the particles correlates to the solar cycle, as it is larger at the solar minimum.

\subsubsection{Secondary Cosmic Rays}

Secondary Cosmic Rays are a product of the GCR environment created from HZE ions colliding with other particles creating fragmentation pieces. This is possible due to the high ionization power of the HZE particles that have sufficient enough energies 


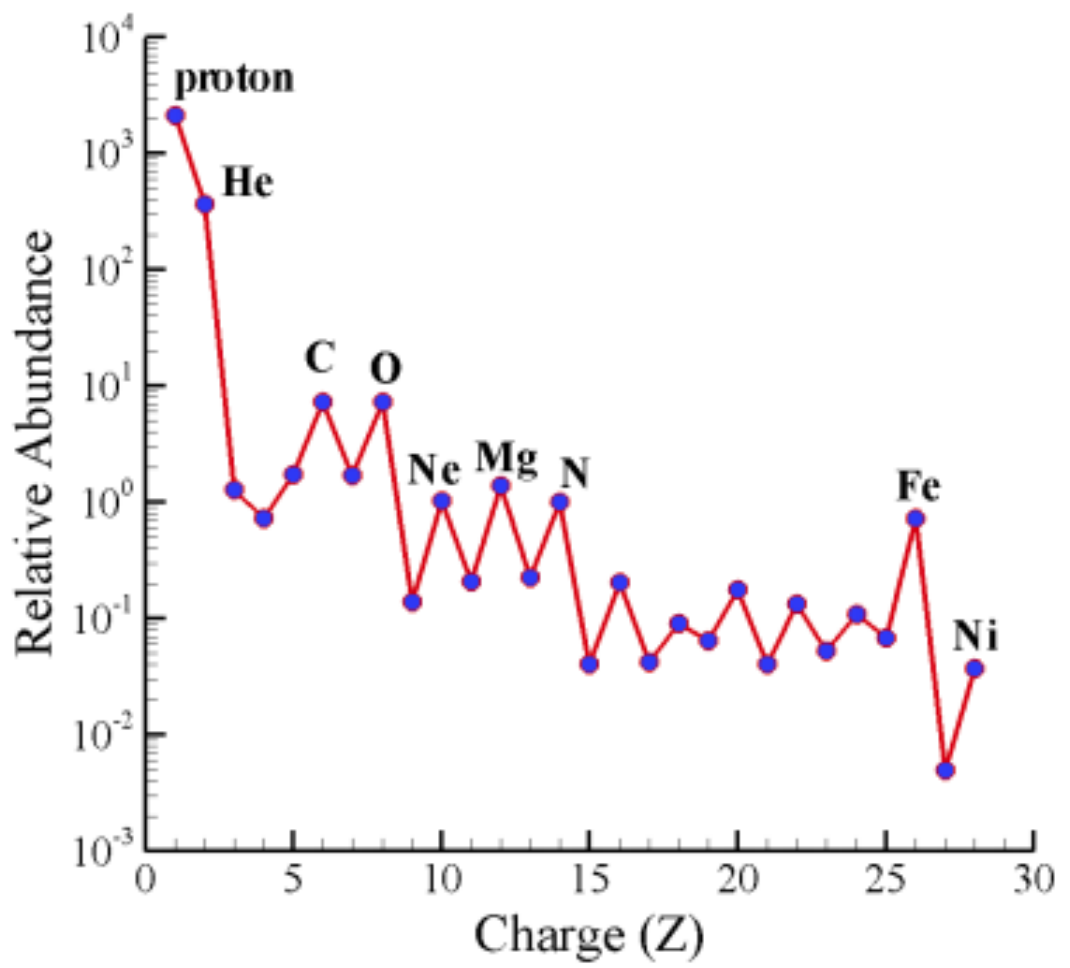

Figure 2.3: Abundance of elements in the GCR environment (1977)[19]

to break apart nuclei[24]. This becomes a concern when shielding for GCRs, as the shield material can produce secondary rays to magnify the total amount of radiation astronauts are exposed to. Often, the fragmentations become more of a problem than the heavy ions themselves due to the low fluence of HZE particles in the GCR environment. When an HZE particle does collide with spacecraft shielding, it creates a "shower" of secondary rays that can produce far more damage than if there were no shield at all. The effects of secondary cosmic rays make it less beneficial to use lead as a protection method and more beneficial to utilize hydrogen based materials[3].

\subsubsection{Solar Radiation}

The sun is a major producer in the radiation environment. The sun emits a flux of radiation that varies in intensity over time called solar wind. This solar flux follows a 


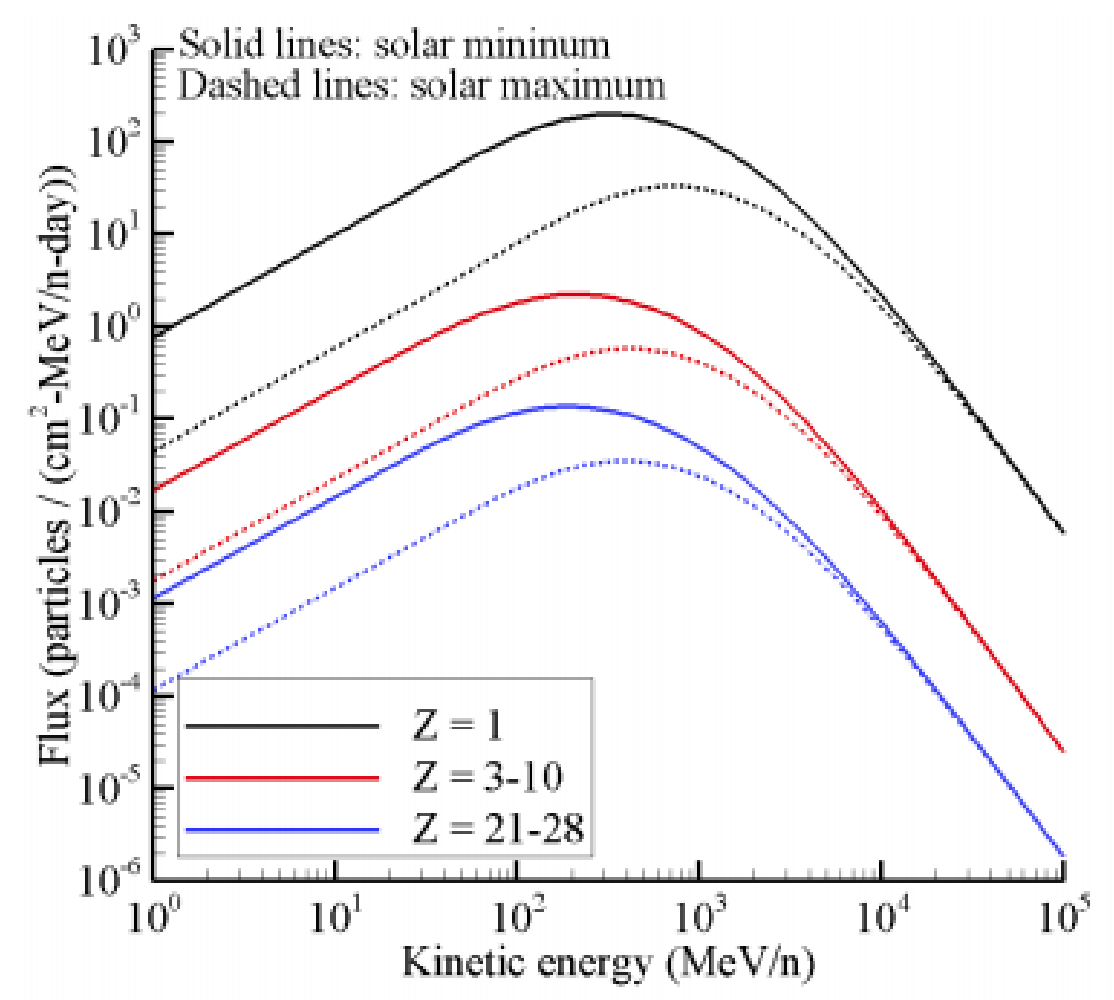

Figure 2.4: GCR flux at solar minima and maxima[19]

cyclical period of around eleven years that results in a minimum and maximum period of solar flux. The solar wind consists of a high density of electrons and protons. In addition, approximately $8 \%$ of the solar wind makeup is alpha particles, and there are trace amounts of heavy ions[15]. Alpha particles consist of helium nuclei, which do not contain the energy to penetrate human skin[11]. The overarching effects of solar wind for humans is negligible, as the particles are easily absorbed by spacecraft shielding[11]. However, the solar cycle is quite important when dealing with more harmful aspects of the radiation environment.

In addition to the solar flux, there are other solar events that add to the chaos of the radiation environment to a much more extreme degree called solar particle events. 


\subsubsection{Solar Particle Events (SPE)}

SPE consists of solar flares and coronal mass ejections. These events vary in intensity, but are much more threatening than the solar wind, and can be an 2-5 times greater in order of magnitude than Galactic Cosmic Rays (GCR)[24]. The radiation consists mostly of high energy protons for a relatively short duration of time. The worst documented events have radiated for several days, including the Carrington event, which is the worst event in recent history[23]. The Carrington event of 1859 is documented to have lasted for two days at high-intensity levels. This event will be detailed in more detail in the following sections.

SPEs are more likely to occur during the solar maximum of the solar cycle, however this does not necessarily indicate the magnitude of the event. Some of the most powerful documented SPEs have occurred at solar minimum adding to the unpredictability of the radiation environment[3]. In addition, the solar events are directionally emitted from the sun. This requires a "perfect storm" for astronauts to be endangered, however, it is likely for long-duration missions. To put into perspective, The Curiosity Rover spacecraft transiting from Earth to Mars in 2011 recorded five separate considerable events[3].

Solar flares occur from explosions due to the tangled magnetic fields of the sun. An image of the surface of the sun revealing the chaotic nature of the magnetic fields can be seen in Figure 2.6[3]. These typically occur over sunspots, where magnetic fields extrude out from the sun. Sunspots are more likely to occur during a solar

maximum and follow the 11-year solar cycle[11]. Light from the flare reaches Earth in 8 minutes, and high energy protons follow at near speed-of-light velocity reaching in as fast as ten to twenty minutes[21]. This leads to high energy collisions Solar flares last from minutes up to hours, ejecting massive quantities of energy to space. Figure 2.5 shows SPE measured over two partial and one entire solar cycles. Plotted 
events indicate a flux greater than $100 \mathrm{MeV}$ protons that penetrate shielding.

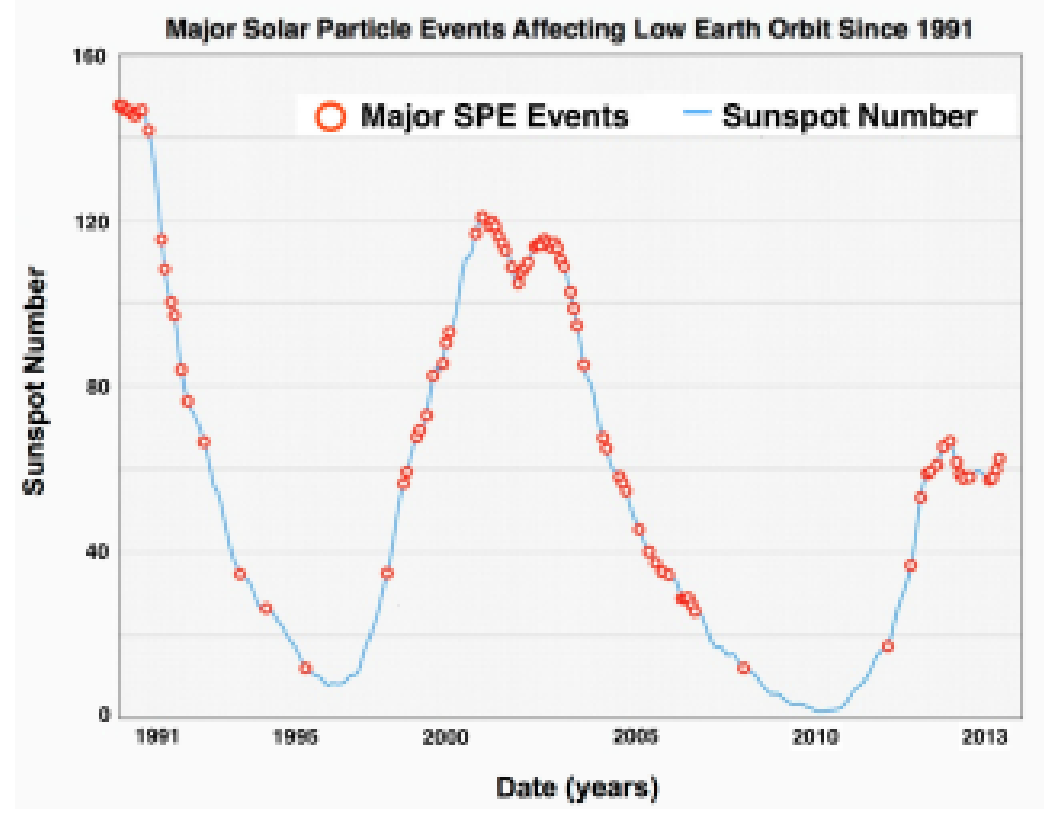

Figure 2.5: Major Solar Particle Events[3]

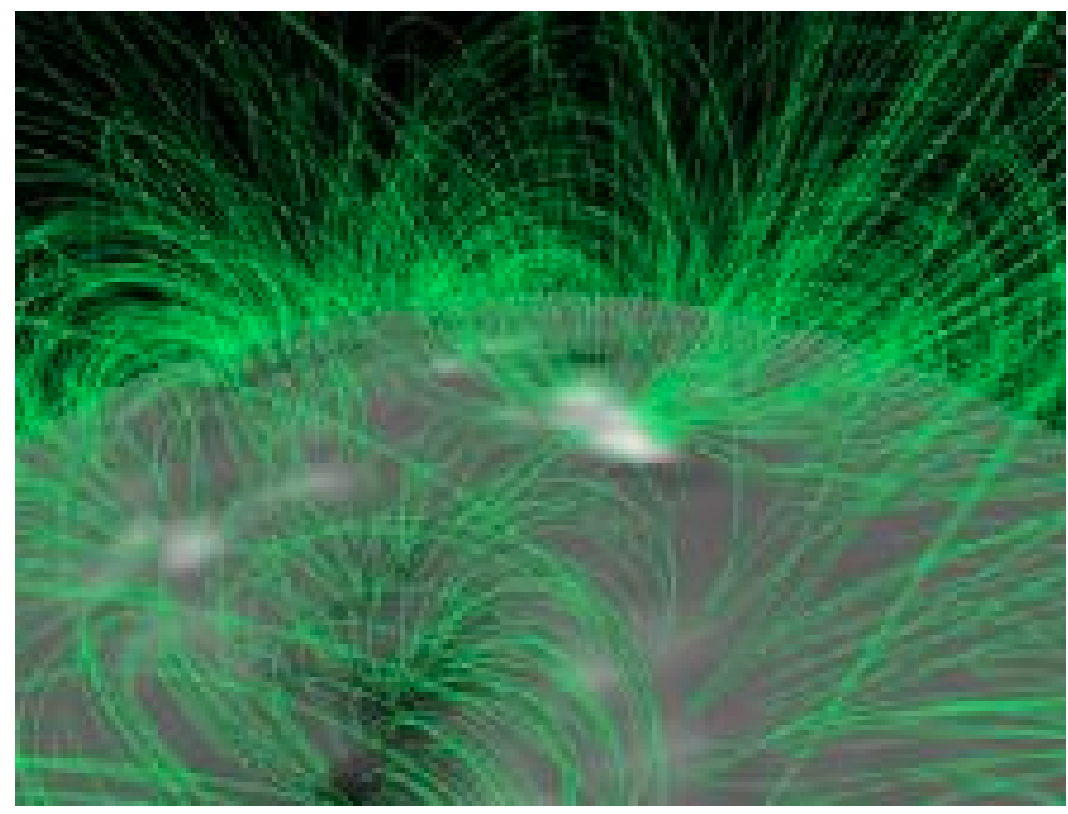

Figure 2.6: Solar magnetic fields [21]

Coronal Mass Ejections (CME) are a dense cloud of magnetized particles that are ejected from the sun. Like solar flares, this release of energetic particles is a 
product of solar magnetic fields and is predominantly electrons and protons. CMEs take considerably longer to reach the Earth, from one to three days[9]. The high intensity nature of these events makes shielding vital, and it is important to give astronauts warning as early as possible to prepare for the increased radiation. Over the last several decades, events have occurred and been recorded. Figure 2.7 overlays major events that took place in the $20^{\text {th }}$ Century. Larger events almost always consist of both a solar flare and a CME, leading to a multitude of energetic particles. Over the years, NASA has undergone tests to the improve upon the warning time for SPEs for astronauts in space. There is currently a warning time on the order of tens of minutes for astronauts to prepare for a storm.

Figure 2.7 breaks down 6 major SPE that have occurred in the last 75 years. It is important to note the unpredictability of a solar storm, as they vary drastically in proton kinetic energy versus fluence. The October 1989 event has the highest fluence of all the major storms, whereas the event in Febuary 1956 had much higher energy per particle. This variability adds to the hostility of the radiation environment. Each of these events will be described in detail in the following sections.

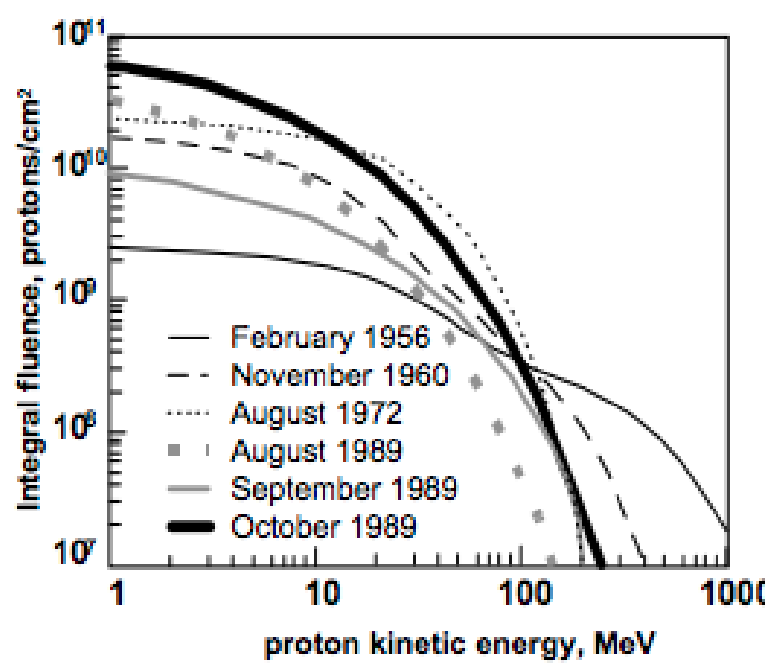

Figure 2.7: Solar Particle Events [24] 


\subsection{Spacecraft in Radiation Environment}

As missions continue to be planned outside of the protection of the Earth's magnetic field, new developments are required to enable safer passage through the radiation environment. Manned missions require even more ingenuity to maintain low dosage levels over a longer mission duration. There are different shielding approaches designed to take on the harsh radiation environment. There are two schools of thought regarding radiation shielding: active methods and passive methods. These different approaches are currently being investigated in industry to best protect astronauts.

\subsubsection{Active Shielding}

Active shielding is similar to the effect of the magnetic belts surrounding the Earth. The belts protect life on the planet from harmful particles. Active shielding techniques have been proposed to create a magnetic field around the spacecraft. This technique has many positive aspects, however, it raises other difficult issues. One is the negative

effects of mixing magnets with electronics[30]. In the small confines of a spacecraft, it would be hard to ensure the safety of the on board electronics. Another is the large power requirement that is demanded from maintaining a magnetic shield around the crew. In addition, the mass of an active shielding system is considerable due to all of the coils, power sources, cooling systems, and support structures required. Currently, active shielding fails to to provide a better level of protection than an equivalent mass of passive shielding.[30]. Active shielding has several hurdles to reach its potential as a shielding technique, but if possible, could prove to be the best option in the future. 


\subsubsection{Passive Shielding}

Passive shielding is the current shielding option used in space. It utilizes the shield to absorb radioactive particles, requiring a certain thickness of material to protect the payload. Passive shielding is limited to material type, thickness, and configuration. The limiting factor of passive shielding comes down to the mass required for thicker

shields. With tight mass budgets there is a limit in thickness, which leads to the necessity of optimizing the shielding configuration to best protect the astronauts as well as electronics on board. The remainder of this paper will be solely focused on passive shielding techniques.

\subsection{Humans}

Humans traveling beyond the Earth's magnetosphere creates a large logistical issue beyond the preexisting electrical problems. Astronauts have a lifetime radiation limit determined by age and sex that gives a ceiling exposure on a long duration mission. In addition to this, there are short-term radiation limits to prevent noncancer health issues including performance degradation, acute radiation sickness, and possibly death[6]. Longer duration missions outside of the Van Allen belts push the boundaries of astronaut radiation limits. NASA has identified four major health risks from radiation: Carcinogenesis, degenerative tissue effect, Central Nervous System (CNS) decrements, and Acute Radiation Syndrome[3]. Cancerous effects are, as expected, a large concern and driving factor when setting career limits for astronauts. However, it is also imperative to ensure astronauts do not receive large short-term dosages that could lead to serious non-cancerous health effects.

NASA currently uses the quantity Risk of Exposure-Induced Death (REID) to determine the risks for astronauts. A REID probability of $3 \%$ is the threshold currently 
set for current ISS and future Moon and Mars exploration missions[5]. This gives the astronauts a $3 \%$ chance of developing cancer in their lifetime, and less than a 1 in 33 chance of early death. This risk factor sets the standard for setting radiation limits for men and women astronauts at different ages using estimates above the $95 \%$ Confidence Level (CL) for uncertainties in risk projection models[5]. Uncertainties occur related to predicting particle energy spectra, and the limited understanding of heavy ion radiobiology leads to a level of uncertainty that requires extra margin when setting radiation limits.

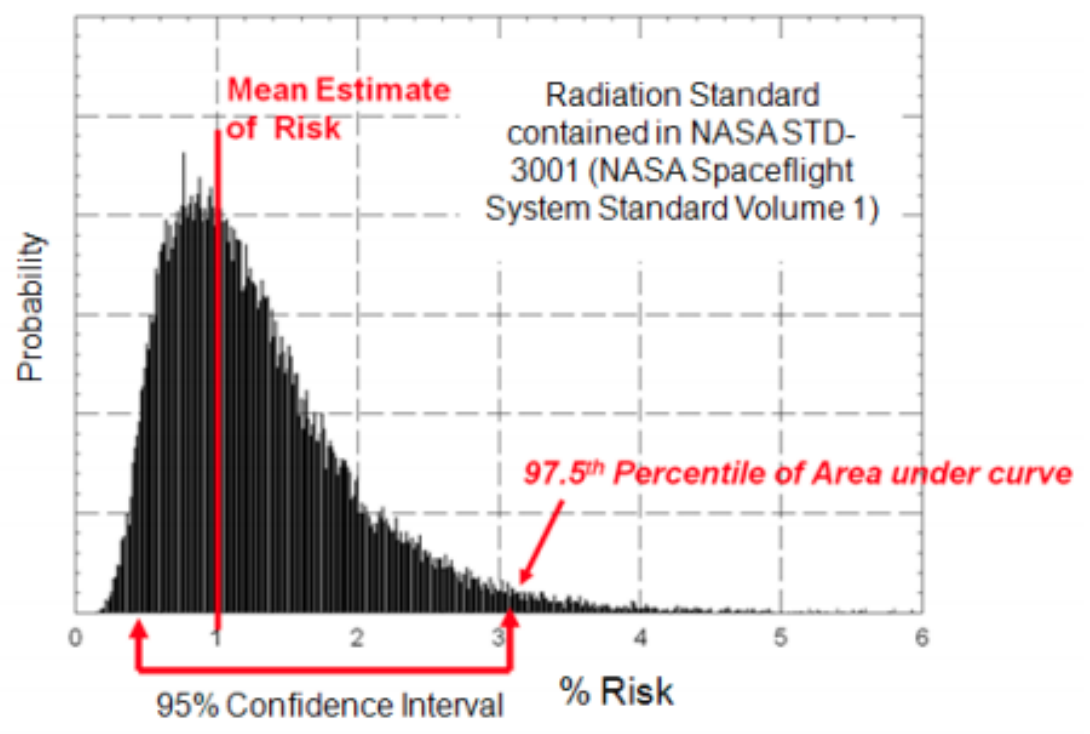

Figure 2.8: NASA Permissible Exposure Limits[25]

The NASA limits are both gender and age specific. The younger the astronaut, the lower the limit. This is from the presumption that exposure early in a career will result in a higher chance of developing health issues during older ages. In addition, radiation limits for women is considerably lower than for men of the same age as seen in Figure 2.9.

The exposure limits in Figure 2.9 are given in units of Sieverts. A Sievert is a unit of radiation dosage measuring the stochastic health risk from the effective dose on a 
human. Effective dose is a measurement of the overarching health of a human. This is calculated through a weighted sum of doses in all the specified tissues and organs of the human body, with a result in Sieverts.

\begin{tabular}{|c|c|c|c|c|}
\hline \multicolumn{5}{|c|}{ Career Exposure Limits for NASA Astronauts by Age and Gender* } \\
\hline Age (years) & 25 & 35 & 45 & 55 \\
\hline Male & $1.50 \mathrm{~Sv}$ & $2.50 \mathrm{~Sv}$ & $3.25 \mathrm{~Sv}$ & $4.00 \mathrm{~Sv}$ \\
\hline Female & $1.00 \mathrm{~Sv}$ & $1.75 \mathrm{~Sv}$ & $2.50 \mathrm{~Sv}$ & $3.00 \mathrm{~Sv}$ \\
\hline
\end{tabular}

Figure 2.9: NASA Radiation Limits[22]

The NASA Space Cancer Risk model was developed for ISS crewed risks and studies for future missions. The model was reviewed by the National Research Council in 2012 and is the most recent iteration used for risk of carcinogenesis in astronauts[25]. There have been several iterations, and although Figure 2.9 gives a good indication of the limit discrepancies between age and gender, the dosage level is outdated. A more recent report from NASA Council on Radiation Protection (NCRP Report 132) has included revised human epidemiology findings to alter limits drastically[5]. This is shown in Figure 2.10, as the updated limits are slashed by more than two-fold. NASA updated to this new standard once it was determined the previous standard was too liberal when considering the stochastic health of the astronauts. The remainder of this study will report on the most updated version of NCRP Report No. 132.

\begin{tabular}{ccccc}
\hline & \multicolumn{2}{c}{ E(Sv), NCRP Report No. 98 } & \multicolumn{2}{c}{ E(Sv), NCRP Report No. 132 } \\
\hline Age at Exposure, & Female & Male & Female & Male \\
$\mathbf{y}$ & & & & 0.7 \\
$\mathbf{2 5}$ & 1.0 & 1.5 & 0.4 & 1.0 \\
$\mathbf{3 5}$ & 1.75 & 2.5 & 0.6 & 1.5 \\
$\mathbf{4 5}$ & 2.5 & 3.2 & 0.9 & 3.0 \\
$\mathbf{5 5}$ & 3.0 & 4.0 & 1.7 & \\
\hline
\end{tabular}

Figure 2.10: NASA Radiation Limit Comparison[5]

Figure 2.11 shows the difference between NASA limits and the general public, while also showing differences in dosages per organ. Astronauts have a higher limit 
due to healthy population characteristics (i.e. Never Smokers).

\begin{tabular}{|c|c|c|c|c|}
\hline \multicolumn{5}{|c|}{$\begin{array}{c}\text { Depth of Radlation Penetration and Exposure Limits } \\
\text { for Astronauts and the General Public (in Sv) }\end{array}$} \\
\hline \multirow{3}{*}{ Astronauts } & $\begin{array}{c}\text { Exposure } \\
\text { Interval }\end{array}$ & $\begin{array}{c}\text { Blood Forming Organs } \\
(5 \mathrm{~cm} \text { depth) }\end{array}$ & $\begin{array}{c}\text { Eyes } \\
(0.3 \mathrm{~cm} \text { depth) }\end{array}$ & $\begin{array}{c}\text { Skin } \\
(0.01 \mathrm{~cm} \text { depth) }\end{array}$ \\
\hline & 30 Days & 0.25 & 1.0 & 1.5 \\
\cline { 2 - 5 } & Annual & 0.50 & 2.0 & 3.0 \\
\cline { 2 - 5 } & Career & $1-4$ & 4.0 & 6.0 \\
\hline \multirow{2}{*}{ General Public } & Annual & 0.001 & 0.015 & 0.05 \\
\hline
\end{tabular}

\section{Figure 2.11: NASA Radiation Penetration and Exposure Limits[22]}

It is critical to protect astronauts from reaching career limits in longer-duration deep space missions, as well as ensure short-term limits are also not exceeded. Figure 2.11 gives an example of short-term limits with 30 day exposure limits. Short-term limits are critical to preventing acute radiation syndrome and other negative noncancer health effects including performance degradation, sickness, or death. The dose limits for the blood forming organs (BFO) are considered adequate measurements to project against the risks of prodromal effects from acute radiation syndrome such as nausea, vomiting, and fatigue[6]. This is reported in Figure 2.11 as 0.25 Sieverts for astronauts, and is not differentiated by age or gender. 


\section{Chapter 3}

\section{OLTARIS}

OLTARIS is the Online Tool for the Assessment of Radiation in Space. It is an International Traffic in Arms Regulations (ITAR) protected NASA tool used to test the effects of space radiation on various shielding materials. The tool's transport and physics is based on the most updated version of HZETRN and NUCFRG2 research codes[28]. The program is constantly under a verification process and tested by NASA to provide the highest possible level assessments for radiation exposure in space applications. This thesis utilizes OLTARIS as the sole tool for testing.

The general process of OLTARIS will be detailed in the following sections to show the program's methodology. Then the calculation process will be discussed with the transport codes described in detail.

\subsection{General Background}

OLTARIS requires users to register for access to the ITAR protected tool. The program is controlled by NASA in order to regulate the limited computer resources[28]. Once the account is activated users can begin creating projects and testing cases. There are several tabs on the home page for Projects, Uploads, Slabs \& Spheres, and Materials.

Projects are the complete package of a calculation enabling the radiation environment, test specimen, and desired responses to be selected by the user. Once the project is submitted to the OLTARIS computer cluster as a job, it is calculated and the designated responses are returned to the user. All of the pertinent response options will be explained in detail in the following sections. 
Uploads is a drop down tab that allows users to select "Thickness Distributions" or "Trajectories". The Thickness Distributions page allows users to upload their own specific vehicle geometry to be tested. This allows users to submit their own CAD models to OLTARIS as a thickness distribution file. The file is required to be in the Extensible Markup Language (XML), which is a markup language in a textual data format. An XML file can be a specifically formatted text file that is then read directly into OLTARIS. OLTARIS also offers a series of basic and/or common geometries that are pre-loaded onto the website. The trajectories allows a user to import a specific trajectory to test radiation dosages for a mission profile.

The Slabs \& Spheres tab allows users to create specific cases and customize the material used. Any sphere can be customized with multiple layers of variable thickness. This gives the user customization without the necessity to convert a CAD file into an XML file thickness distribution. In addition, each layer within a user-defined sphere has a drop-down option to determine the material. OLTARIS has a variety of pre-defined materials, or the user can create a new material.

The Material tab shows users which materials are available and allows additional materials to be uploaded. Materials within OLTARIS include aluminum, aluminumlithium, water, polyethylene, tissue, silicon, Mars regolith, and Moon regolith. New materials can be defined through elemental or molecular mass percentage, or through a chemical formula.

\subsection{OLTARIS Calculation Process}

Once the user defines the environment, the test geometry through a thickness distribution, and the desired responses, OLTARIS runs calculations to determine the dosages from the radiation environment. This is done through several transport codes that will be explained in more detail in the following subsections. The process is described 
in Figure 3.1 with a flow diagram. The user defines the testing parameters and submits the project, then OLTARIS implements the transport codes and calculates the desired response function to output to the user.

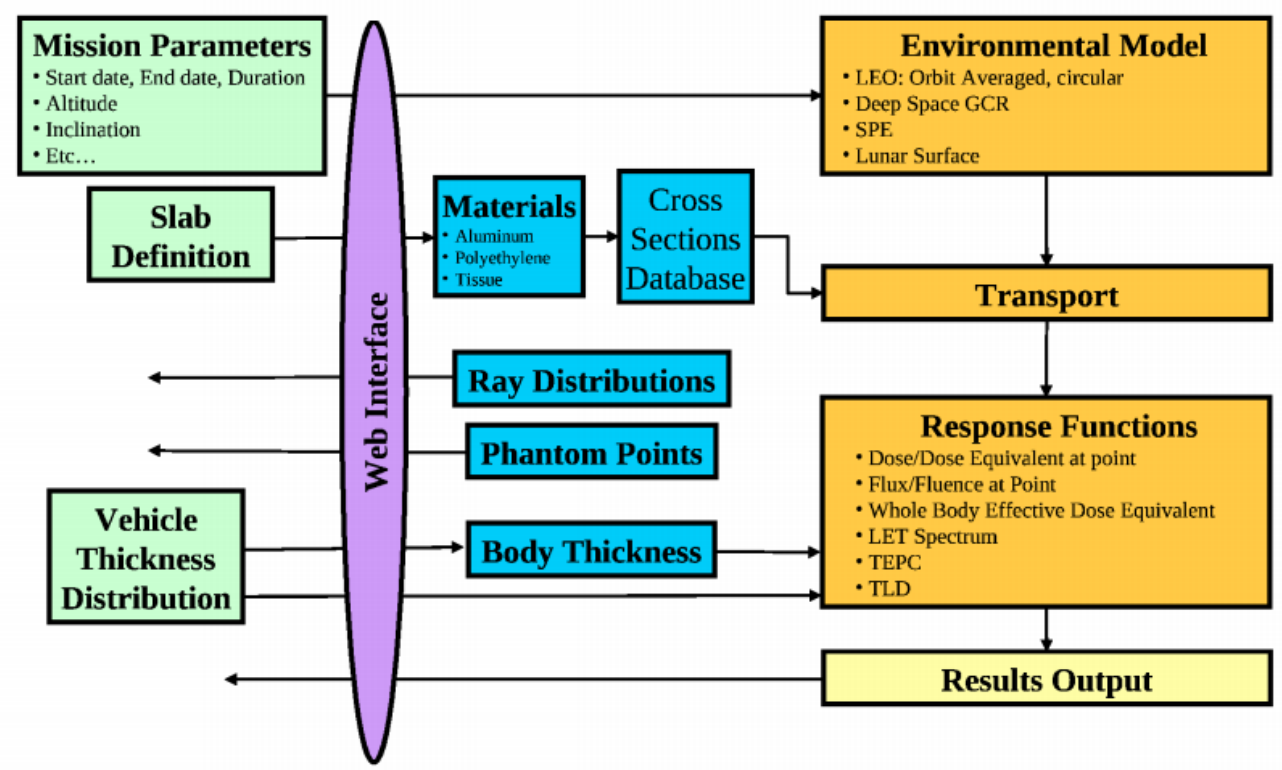

\section{Figure 3.1: OLTARIS Program Flow[28]}

OLTARIS runs with two main codes for particle transport calculation, HZETRN2005 with NUCFRG2. The transport solution will be detailed further below to describe the calculation process taken for both light ions $(A \leq 4)$, which includes neutrons, and heavy ions $(A \geq 4)$ where $\mathrm{A}$ represents the atomic mass number of the particles.

\subsubsection{HZETRN2005}

The High charge (Z), and Energy TRaNsport code (HZETRN2005) was developed at the Langley Research Center as part of the NASA strategic plan for human exploration of space[12]. HZETRN is a code used for radiation analysis under a variety of conditions including Galactic Cosmic Rays and Solar Particle Events. This code is consistently revised, verified, and validated as new data is discovered. This has led to a fluid transport code that is updated and adapts over the past several decades. 
HZETRN works through a numerical marching procedure to determine the first deterministic solution to the Boltzmann equation. A marching solution is a numerical method to solving boundary value problems. The linear Boltzmann equation is commonly used to model many systems, including neutronic dynamics, and radiation transfer.

The linear Boltzmann equation is depicted below in Equation 3.1 with the Continuous Slowing Down Approximation (CSDA) and straight ahead approximation. This marching procedure is sufficient to boundary conditions found in the radiation environment enabling validation to take place for decades using the Space Transport System (STS) and the International Space Station (ISS) [31].

$$
\left.\left.\left[\frac{\partial}{\partial x}-\frac{1}{A_{j}} \frac{\partial}{\partial E} S_{j}(E)+\sigma_{j}(E)\right)\right] \phi_{j}(x, E)\right)=\sum_{k} \int_{E}^{\infty} d E^{\prime} \sigma_{k \rightarrow j}\left(E^{\prime} \rightarrow E\right) \phi_{k}\left(x, E^{\prime}\right)
$$

where $S_{j}(E)$ is the stopping power with kinetic energy, $E . \phi_{j}(E)$ is the total macroscopic cross section, and $\sigma_{k \rightarrow j}\left(E^{\prime} \rightarrow E\right) \phi_{k}\left(x, E^{\prime}\right)$ is the macroscopic production cross section for particle interactions. The boundary condition used for the Boltzmann equation is in Equation 3.2 where $\sigma_{j}(x, E)$ is the flux of type $j$ particles at depth $x$ with kinetic energy $E[28]$.

$$
\sigma_{j}(0, E)=f_{j}(E)
$$

There are several assumptions made to create the boundary value problem as mentioned earlier. The CSDA assumes that atomic interactions occur at a significant enough pace that the process can be modeled as continuous. The straight ahead approximation assumes that primary and secondary particles propagate in the same direction in order to simplify the calculation process to a one dimensional space 
without angular considerations.

The particle source is given through the boundary condition allowing a marching algorithm to be implemented to solve the equation in any particular direction. Heavy ion transport is described in the version of the Boltzmann equation shown in Equation 3.3. It is deemed valid by NASA to assume that heavy ion projectiles and target fragments have equal velocity for simplification in heavy ion transport[28].

$$
\left.\left.\left[\frac{\partial}{\partial x}-\frac{1}{A_{j}} \frac{\partial}{\partial E} S_{j}(E)+\sigma_{j}(E)\right)\right] \phi_{j}(x, E)\right)=\sum_{k>j} \int_{E}^{\infty} d E^{\prime} \sigma_{k \rightarrow j}\left(E^{\prime} \rightarrow E\right) \phi_{k}(x, E)
$$

For light ion and neutron calculations, it is not fair to assume projectiles and target fragments have equal velocity for simplification. This differs from heavy ion calculations. Equation 3.1 is utilized where the summation takes into account all light particles.

\subsubsection{NUCFRG2}

The NUClear FRaGmentation database (NUCFRG2) is used by OLTARIS for heavy ion cross sections in tandem with OLTARIS[28]. NUCFRG2 is a model based on an abrasion-ablation concept of secondary rays, created by large heavy ionizing particles that makeup the GCR spectrum colliding with shielding material. This creates an abrasion, in which the particle enters a highly excited state. This leads to decay, in which the piece ablates and emits energetic particles. This process is determined within NUCFRG2 by considering the relative impact parameter of a colliding spherical nuclei[28].

NUCFRG2 is sufficient at handling Heavy Ion Based Nuclear Cross Sections, however is limited for nucleon and light ion projectiles. These calculations are 
handled outside NUCFRG within several other OLTARIS modules. Analysis includes the effects of elastic scattering, light ion projectile fragmentation, and target fragmentation[28].

\subsubsection{Restrictions}

NUCFRG2 has inherent issues from assumptions made for calculations. The code neglects quantum mechanical effects of the particles and omits critical shell structure information. This leads to an effect in which the cross sections for fragments with an even number of nucleons is much larger than those with an odd number. This is related directly to the nuclear pairing interaction and the odd-even staggering in binding energies[28]. OLTARIS is currently attempting to upgrade this for future updates, however the results discussed in this project will be limited to current versions of the code.

The radiation environment has been a new study and is relatively unknown. There is little data collected over the few decades we have had access to deep space sensors. This has lead to tools with accuracy limited by the lack of data available to work with and levels of uncertainty. For this reason, there is no known error for results produced within OLTARIS.

\subsection{Thickness Distributions}

OLTARIS requires a representation of the vehicle's geometry through a material thickness distribution. The thickness distribution is calculated through a process called ray tracing. This is where a directionally distributed set of rays determine the thickness of materials that surrounds the point from which the rays originate[28]. This point of origin is called the target point. OLTARIS requires a specific XML format for the thickness distribution as seen in Figure 3.2. In order to formulate this file, a 
separate tool is required outside of OLTARIS. This process will be detailed in further sections. The file must dictate the material units, the type of material, and the thickness eminating from each ray for each material. With this, OLTARIS can calculate and determine the effects of radiation observed from an astronaut in space.

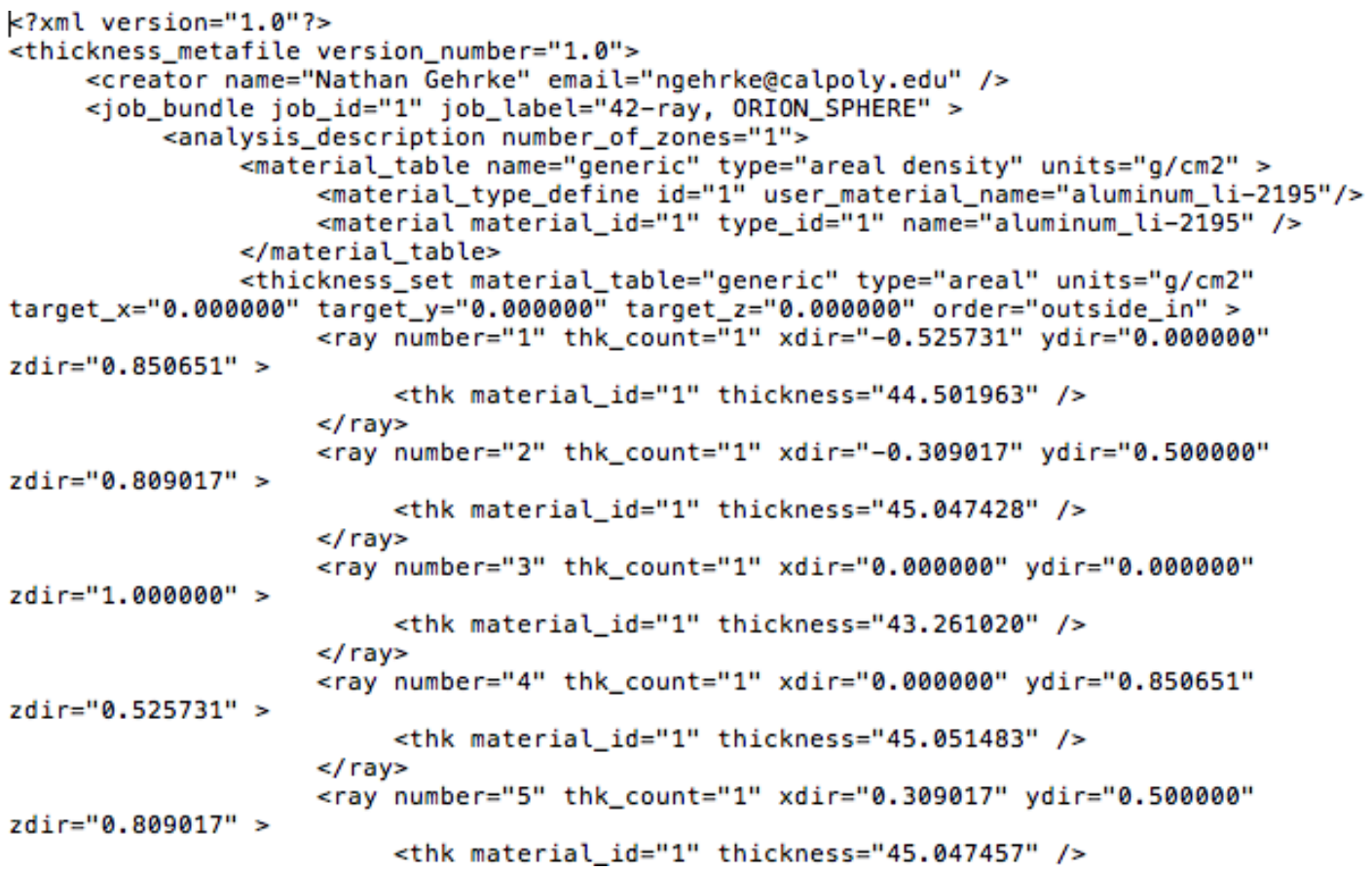

Figure 3.2: Example Thickness Distribution

In order for the job to compute human doses, OLTARIS requires ray distributions with $42,492,512,968,1002,4002,9002$, or 10,000 rays. The more rays used, the higher fidelity the results, however, the computing time increases drastically. This relationship between the various ray distribution files will be detailed further in chapter 5. The files can be downloaded from the web interface by the user. The geodesic ray distributions create an equally spaced ray distribution and are ideal options for testing in this analysis. Geodesic ray files include the 42, 492, 1002, 4002, and 9002 ray options as seen in Figure 3.3.

OLTARIS has a default unit of areal density in $\mathrm{g} / \mathrm{cm}^{2}$. This value is material specific and incorporates the density of the material within the thickness. The thickness 


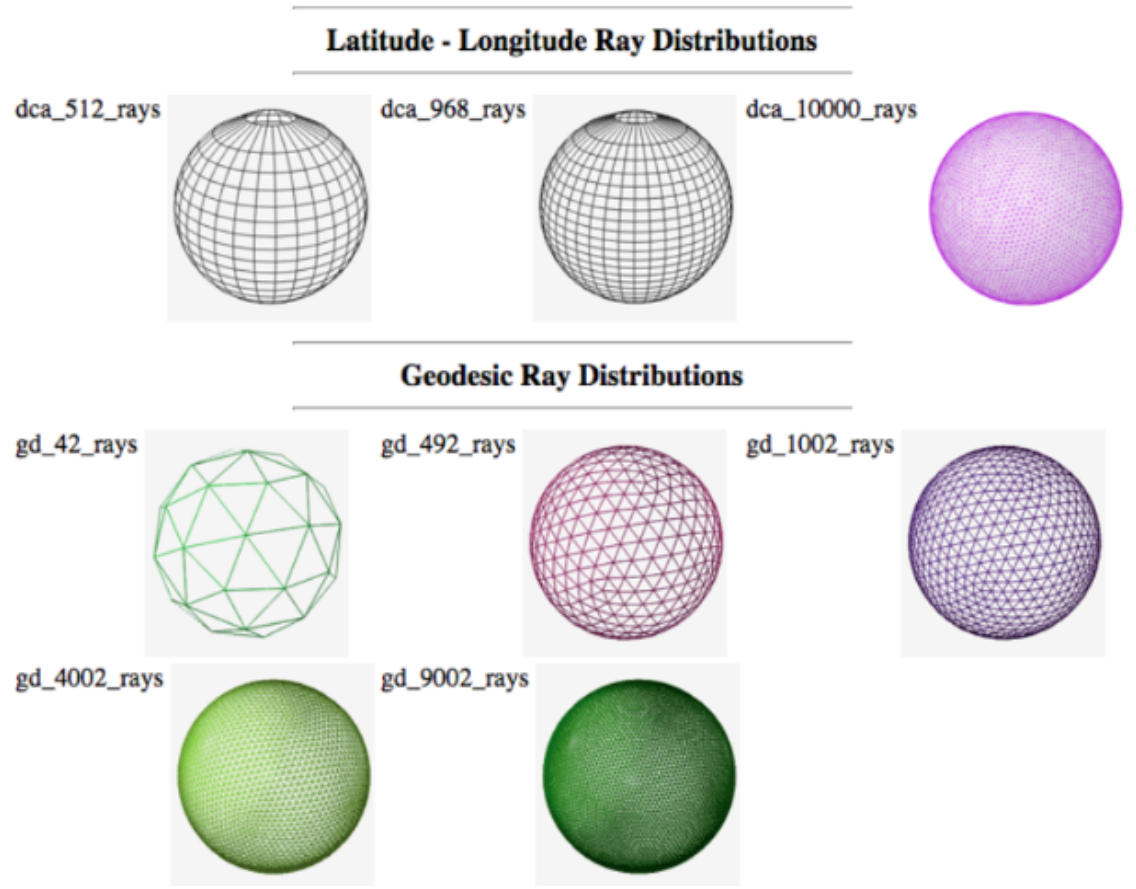

\section{Figure 3.3: Ray Distributions Accessable Through OLTARIS[10]}

distribution must have the units defined, and be uniform throughout the document. OLTARIS also supports $\mathrm{cm}$, in, and $\mathrm{kg} / \mathrm{m}^{2}$ as acceptable units.

Additional ray distributions can be downloaded to support the testing of human bodies within the spacecraft, called phantom bodies. These ray distributions are downloaded as rotated ray files from the OLTARIS website, which allows the user to input the body in any location within the vehicle. The user can define the orientation of the phantom body within the vehicle, as well as change the target point.

\subsubsection{Phantom Bodies}

OLTARIS offers CAD of phantom bodies to be placed within the CAD of the test specimen to be used as the test dummies. The Computer Anatomical models are available in both Male (CAM) and Female (CAF) types and include eight reference points. This is shown in Figure 3.4 with the five target zones of the human body 
as well as the three green points used to establish the phantom's orientation in $\mathrm{x}, \mathrm{y}$, and z. The CAD models can be downloaded from OLTARIS and placed within the vehicle model. As mentioned previously, a rotated ray file can be downloaded using the $\mathrm{x}, \mathrm{y}$, and $\mathrm{z}$ phantom body orientation points to create a thickness distribution file for the vehicle with humans included.
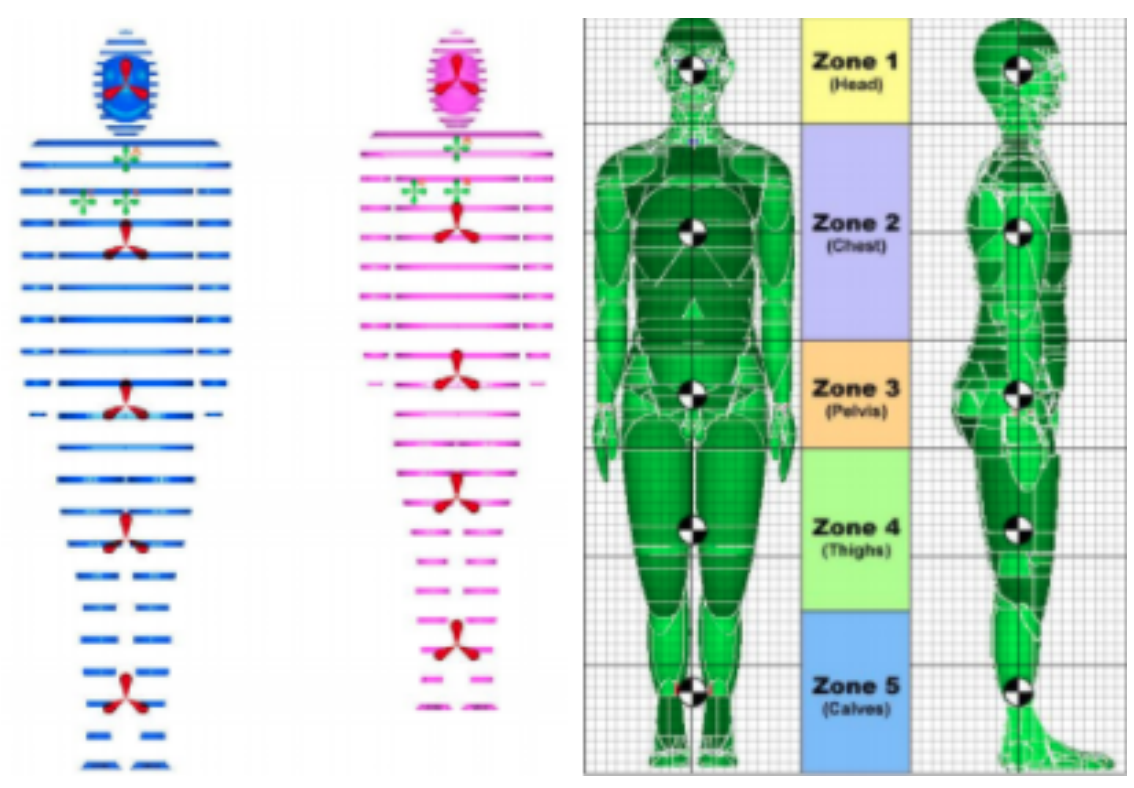

Figure 3.4: Phantom Bodies available for download through OLTARIS[28]

The other five points cover the five body zones of the head, chest, pelvis, thighs, and calves. Each of these points requires separate vehicle thickness distributions. This enables OLTARIS to output high fidelity effective whole-body dose equivalent values. This calculation uses thickness distributions based of a multitude of target points that are distributed throughout phantom body in all of the body zones. These distributions use human tissue as the material to compute dosages. The vehicle thickness distribution can then be added to the tissue distributions. OLTARIS determines the thickness of shielding in the vehicle closest to each of the zones tissue thickness distributions to get the total shielding around each body point[28]. This method is efficient and beneficial to the user, as only one target point is required for the initial vehicle thickness distribution rather than five for each of the body zones shown in 
Figure 3.4.

OLTARIS is limited to three materials when calculating for effective dose equivalent for the human models. One of those three materials is required to be tissue for the OLTARIS calculation, which limits the user to only input two materials to the system for spacecraft shielding. This is a huge limitation, as the Orion capsule used for testing is composed of multiple materials. In addition, the bladder required to hold the water for testing can not be included in radiation analysis when calculation the effective dose equivalent.

\subsection{Material Properties}

The main three materials for OLTARIS are Aluminum, Polyethylene, and tissue. There are additional materials pre-loaded into the tool including water and AluminumLithium, and the ability for the user to import their own material properties. Material properties are very important within the HZETRN algorithm, as material dependant cross sections are used to predict how neutrons and charged ions will interact with the vehicle shield[28].

As mentioned earlier, OLTARIS has the ability to support many materials. However, OLTARIS is limited for the effective dose response calculations.

\subsection{Radiation Environment}

OLTARIS offers radiation data for a variety of locations within the solar system including customized Earth orbits, free space $1 \mathrm{AU}$ from the sun, and the lunar and mars surfaces. There are options to use either the Badhwar-O'Neil 2010 model (BON10), the Badhwar-O'Neil 2014 model (BON14), or the Matthia 2013 model. The latter two models data are valid through January 2017, whereas the former is 
only valid through Dec. 2012. The two models considered for the analysis are the Matthia 2013 model and the BON14 model. Only the BON14 model is considered, as it is a revised version of the 2010 model. It offers higher fidelity results with a larger base of measurements in its foundation. The BON14 and Matthia 2013 model are within 10\% of each other, on average, over past 40 years shown in Figure 3.5[29]. The Matthia 2013 model is a simplified form of the Russian Nymmik semi-empirical model[29]. Although similar in comparison, the Matthia model is not as consistent as the BON14, and is not directly derived from measurements taken in space. All GCR analysis done in this study utilizes the Badhwar 2014 model, which is the default OLTARIS and NASA selection.

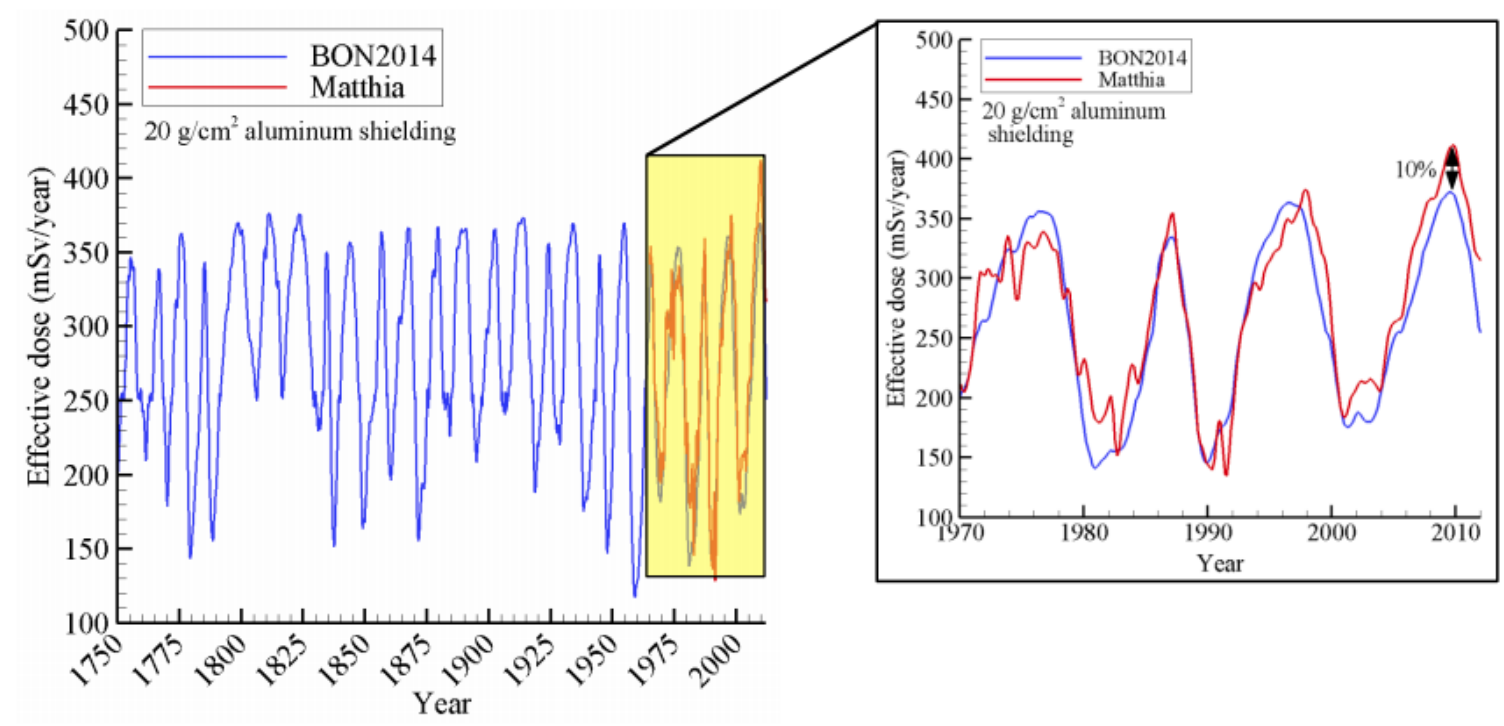

\section{Figure 3.5: OLTARIS GCR Model Comparison[29]}

The Badhwar-O'Neil 2014 GCR model is based on measurements taken from particle detectors in space. The model has mainly been used by NASA to certify electronic systems and the determine the radiation health risks for astronauts[19]. The BON14 model numerically solves the Fokker-Planck (FP) differential equation for particle transport in the heliosphere due to diffusion, convection, and adiabatic deceleration[19]. This is solved with an assumption of a spherically symmetric he- 
liosphere. It also incorporates a time delay function to account for the delay the solar activity has reaching the boundary of the heliosphere[19]. The FP differential equation is shown in Equation 3.4:

$$
\frac{1}{r^{2}} \frac{\partial}{\partial r}\left(r^{2} V_{s} U\right)-\frac{1}{3}\left[\frac{1}{r^{2}} \frac{\partial}{\partial r}\left(r^{2} V_{s}\right)\right]\left[\frac{\partial}{\partial T}(\alpha T U)\right]=\frac{1}{r^{2}} \frac{\partial}{\partial r}\left(r^{2} k \frac{\partial U}{\partial r}\right)
$$

where $\mathrm{r}$ is the radial position in astronomical units $(\mathrm{AU})$; $\mathrm{T}$ is the kinetic en$\operatorname{ergy}(\mathrm{MeV} / \mathrm{n}) ; U(r, T)$ is the GCR flux (particles/srm $\left.{ }^{2} s M e V / n\right), V_{s}(r)$ is the solar wind speed $(400 \mathrm{~km} / \mathrm{s}) ;(r, T)$ is the particle diffusion coefficient tensor; and $\alpha(T)=\left(T+2 T_{0}\right) /\left(T+T_{0}\right)$, with $T_{0}$ being the rest energy of the GCR particle. The solution also assumes that at a boundary distance $r=R_{b}$, modulation of $U(r, T)$ is negligible, and therefore provides the boundary condition at $U\left(R_{b}, T\right)=U_{0}$ as a known value[19]. This value, $U_{0}$, is ion specific and parametrically described by several free parameters[19].

\subsubsection{Historical SPE}

OLTARIS has loaded data for historical SPE, and models available for customization. The available historical events include the Carrington event of 1859, as well as events in 1956, 1960, 1972, and 1989. Each of these events has corresponding explicit differential formulas within OLTARIS. These formulas are portrayed in Equation 3.5 through Equation 3.14. In addition, the events are scalable to allow the user to customize environments. The user also has the option to customize an entire new event,

and import an explicit differential formula into OLTARIS. The events provided by OLTARIS will be described in more detail in chapter 6 to determine the worst-case scenario for analysis. This study will focus solely on historical events $1: 1$ scale. 
February 1956 Webber, with 100 MV rigidity[28]:

$$
\phi(E)=1.0 \times 10^{7}\left[\frac{E+m}{\sqrt{E(E+2 m)}}\right] \exp \left[\frac{239.1-\sqrt{E(E+2 m)}}{100}\right]
$$

February 1956 LaRC[28]:

$$
\phi(E)=6.0 \times 10^{7} \exp \left(\frac{10-E}{25}\right)+9.375 \times 10^{5} \exp \left(\frac{100-E}{320}\right)
$$

November $1960[28]$ :

$$
\phi(E)=6.33 \times 10^{8} \exp \left(\frac{10-E}{12}\right)+4.88 \times 10^{6} \exp \left(\frac{100-E}{80}\right)
$$

August 1972 King[28]:

$$
\phi(E)=2.98 \times 10^{8} \exp \left(\frac{30-E}{26.5}\right)
$$

August 1972 LaRC[28]:

$$
\phi(E)=2.2 \times 10^{7} \exp \left(\frac{100-E}{30}\right)
$$

September $1989[28]$ :

$$
\phi(E \leq 10 \mathrm{MeV})=1.446 \times 10^{8}\left[\frac{E+m}{\sqrt{E(E+2 m)}}\right] \exp \left[\frac{-\sqrt{E(E+2 m)}}{102.118}\right]
$$

$\left.\phi(10 \mathrm{MeV} \leq E \leq 30 \mathrm{MeV})=-0.0015 E^{2}+0.07184 E+0.4304\right] \phi(E \leq 10 \mathrm{MeV})$

$$
\phi(E \geq 30 \mathrm{MeV})=\frac{2.034 \times 10^{7}}{\sqrt{1-\left(\frac{m}{E+m}\right)^{2}}}\left[\sqrt{\frac{E(E+2 m)}{30(30+2 m)}}\right]^{-4.5}
$$

October $1989[28]$ :

$$
\phi(E)=6.104 \times 10^{8}\left[\frac{E+m}{\sqrt{E(E+2 m)}}\right] \exp \left[\frac{-\sqrt{E(E+2 m)}}{92.469}\right]
$$


Carrington $1859[28]$ :

$$
\phi(E)=0.972 \times 0.441 E^{.441-1} \times 1.47 \times 10^{12} \exp (-0.972 E 0.441)
$$

The Carrington event is the largest solar storm in recent history dating back to the past 450 years[23]. The data used for the Carrington event is created by a mathematical model since it occurred over a century before its magnitude could be measured. The model was created based on data taken from a Greenland ice core, which can determine the magnitude of a solar storm[23]. The ice core provides valuable information for solar storms through ozone depletion. This allows the ability to create detailed models for historical solar storms. The $>30 \mathrm{MeV}$ solar proton fluence determined from the ice cores indicate it was double the magnitude of the next largest event (1895), and approximately four times larger than the solar proton fluence of the largest event from the spacecraft era[23]. The total ionization occurred over a 2 day duration uniformly, leading to a localized maximum column ozone depletion which was 3.5 times greater than that of the 1989 event[23]. The Carrington event of 1859 is modeled with a soft fit of the October 1989 event within OLTARIS. A screen capture from video of a model of the 1859 Carrington Event is shown in Figure 3.6[17].

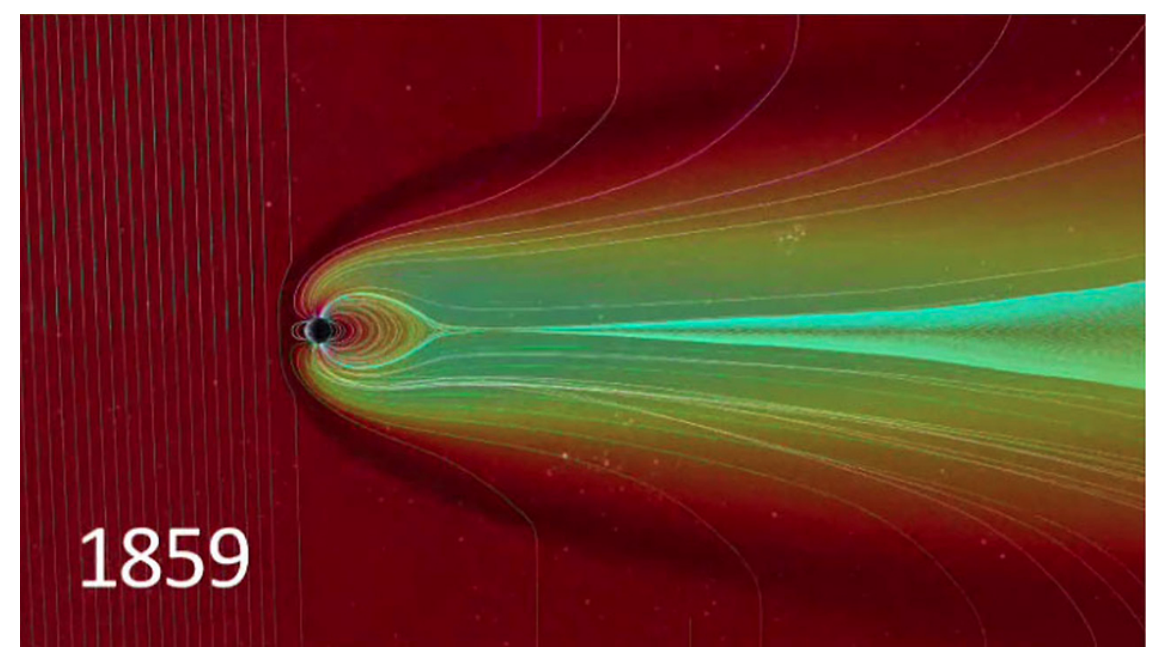

Figure 3.6: Carrington SPE Model[17] 


\subsection{Response Functions}

The flux of the particles within the vehicle are a detailed quantity that describe the environment, however does little to quantify the damage seen by humans or materials throughout the mission. OLTARIS utilizes other functions to display more meaningful data for the user. Once the flux spectrum is calculated by OLTARIS with HZETRN and NUCFRG2, the data is modified within the program to output the user selected response functions. There are a number of available response functions available to select including dose, dose equivalent, effective dose equivalent, and Linear Energy Transfer (LET).

\subsubsection{Dose}

Dose is a basic response function calculated by OLTARIS from determining the energy deposited along each particle's track traveling through the spacecraft. This is shown in Equation 3.15, where $D_{j}$ is defined in Equation 3.16.

$$
\begin{gathered}
D=\sum_{j} D_{j} \\
D_{j}=\int_{0}^{\infty} d E S_{j}(E) \phi_{j}(E)+d^{*}(E)
\end{gathered}
$$

where $S_{j}(E)$ is the stopping power of a charged particle at energy, E, in the material. In this study, the material of interest is tissue for the astronauts in the mission. As neutrons do not interact electromagnetically, they are not included in calculations of stopping power. Other neutron interactions are ignored. Equation 3.17 is used if the stopping power of a proton is not known. Equation 3.17 serves as an accurate method to determine the stopping power and does not compromise the results. Often the value should be known unless using a user-defined material within 
OLTARIS. The $d^{*}(E)$ term in the equation accounts for the target fragments and recoil nuclei not transported[28].

$$
S_{j}(E)=\frac{z_{j}^{2}}{A_{j}} S_{\text {proton }}(E)
$$

Dose is given in units of milligray, mGy. It is calculated and given to the user in dose tables or dose versus depth tables, which can be interpolated to determine the dose at a target point within the vehicle.

\subsubsection{Dose Equivalent}

Dose gives the energy deposited on a material from the radiation environment, but is not as useful for determining effects in humans from dosages. Dose is often used for energy deposited on electronics for both uncrewed and crewed missions. When considering the complicated mixture of high-and low-LET radiation, the National Council on Radiation Protection and Measurement endorses the use of dose equivalent calculated with the ICRP-60 quality factor[28]. Dose equivalent utilizes this quality factor and is much better at estimating the probability of stochastic effects in humans such as cancer mortality than dose values[28]. Dose Equivalent is shown as $\mathrm{H}$ in Equation 3.18. Equation 3.19 defines the $H_{j}$ term.

$$
\begin{gathered}
H=\sum_{j} H_{j} \\
H_{j}=\int_{0}^{\infty} d E Q_{I C R P-60}\left(S_{j}(E)\right) S_{j}(E) \phi_{j}(E)+h^{*}(E)
\end{gathered}
$$




$$
Q_{I C R P-60}\left(S_{j}(E)\right)=\left\{\begin{array}{c}
0<S_{j}(E) \leq 10=1 \\
10<S_{j}(E) \leq 100=0.32 S_{j}(E)-2.2 \\
100>S_{j}(E)=\frac{300}{\sqrt{S_{j}(E)}}
\end{array}\right.
$$

where $S_{j}(E)$ is the stopping power, which can be calculated as it was for dose with Equation 3.17. The target fragments and recoil nuclei are not transported, so their dose equivalent is added by the $h^{*}(E)$ function. The quality factor $Q_{I C R P-60}$ is defined in Equation 3.20. The units of dose equivalent are displayed in millisieverts, $\mathrm{mSv}$.

\subsubsection{Effective Dose Equivalent}

The effective dose equivalent is calculated through the use of phantom bodies within OLTARIS. The calculation can be done with both male and female models (CAM and CAF). Effective dose is determined after finding the equivalent dose in each tissue and organ of the human model. Those doses are then weighted to determine the total effect on the human body as shown in Equation 3.21

$$
E D=\sum_{T} w_{T} \bar{H}_{T}
$$

where $w_{T}$ represents the tissue weighting factors and $\bar{H}_{T}$ represents the averaged dose equivalents calculated in OLTARIS. Figure 3.7 shows the OLTARIS output with the calculated doses in each tissue of the body to determine the effective dose equivalent. Effective dose equivalent is portrayed in units of $\mathrm{mSv}$, which requires an input effective dose, $\bar{H}_{T}$, in $\mathrm{mSv}$ as well.

This figure is an example and is not the exhaustive list of all the human components considered. Each of these tissues is then weighted and applied to Equation 3.21 


\begin{tabular}{|c|c|c|c|}
\hline Organ & Avg. Dose & Per Day & Per Year \\
\hline Adrenals & $6.645 \mathrm{E}+00 \mathrm{mGy}$ & 3.164E-01 mGy/day & $1.155 \mathrm{E}+02 \mathrm{mGy} /$ year \\
\hline BFO & $6.698 \mathrm{E}+00 \mathrm{mGy}$ & 3.189E-01 mGy/day & 1.164E+02 mGy/year \\
\hline Bladder & $6.609 \mathrm{E}+00 \mathrm{mGy}$ & 3.147E-01 mGy/day & 1.149E+02 mGy/year \\
\hline Bone & $6.759 \mathrm{E}+00 \mathrm{mGy}$ & 3.219E-01 mGy/day & 1.175E+02 mGy/year \\
\hline Brain & $6.814 \mathrm{E}+00 \mathrm{mGy}$ & 3.245E-01 mGy/day & 1.184E+02 mGy/year \\
\hline Breast & $6.823 E+00 m G y$ & 3.249E-01 mGy/day & 1.186E+02 mGy/year \\
\hline Esophagus & $6.808 \mathrm{E}+00 \mathrm{mGy}$ & 3.242E-01 mGy/day & 1.183E+02 mGy/year \\
\hline Heart & $6.648 \mathrm{E}+00 \mathrm{mGy}$ & 3.166E-01 mGy/day & 1.155E+02 mGy/year \\
\hline Hippocampus & $6.790 \mathrm{E}+00 \mathrm{mGy}$ & 3.233E-01 mGy/day & $1.180 \mathrm{E}+02 \mathrm{mGy} /$ year \\
\hline Intestine & $6.712 \mathrm{E}+00 \mathrm{mGy}$ & 3.196E-01 mGy/day & 1.167E+02 mGy/year \\
\hline Kidneys & $6.638 \mathrm{E}+00 \mathrm{mGy}$ & 3.161E-01 mGy/day & $1.154 \mathrm{E}+02 \mathrm{mGy} /$ year \\
\hline Lens & $6.932 E+00 \mathrm{mGy}$ & 3.301E-01 mGy/day & $1.205 \mathrm{E}+02 \mathrm{mGy} /$ year \\
\hline Liver & $6.613 E+00 m G y$ & 3.149E-01 mGy/day & 1.149E+02 mGy/year \\
\hline Lungs & $6.760 \mathrm{E}+00 \mathrm{mGy}$ & 3.219E-01 mGy/day & $1.175 \mathrm{E}+02 \mathrm{mGy} /$ year \\
\hline Muscle & $6.775 \mathrm{E}+00 \mathrm{mGy}$ & 3.226E-01 mGy/day & $1.178 \mathrm{E}+02 \mathrm{mGy} /$ year \\
\hline Pancreas & $6.644 \mathrm{E}+00 \mathrm{mGy}$ & 3.164E-01 mGy/day & $1.155 \mathrm{E}+02 \mathrm{mGy} /$ year \\
\hline Salivaryglands & $6.864 \mathrm{E}+00 \mathrm{mGy}$ & 3.268E-01 mGy/day & 1.193E+02 mGy/year \\
\hline Skin & $6.930 \mathrm{E}+00 \mathrm{mGy}$ & 3.300E-01 mGy/day & $1.205 \mathrm{E}+02 \mathrm{mGy} /$ year \\
\hline Spleen & $6.631 E+00 \mathrm{mGy}$ & 3.157E-01 mGy/day & $1.152 \mathrm{E}+02 \mathrm{mGy} /$ year \\
\hline Stomach & $6.628 \mathrm{E}+00 \mathrm{mGy}$ & 3.156E-01 mGy/day & $1.152 \mathrm{E}+02 \mathrm{mGy} /$ year \\
\hline Thymus & $6.751 \mathrm{E}+00 \mathrm{mGy}$ & 3.215E-01 mGy/day & $1.173 \mathrm{E}+02 \mathrm{mGy} /$ year \\
\hline Thyroid & $6.856 \mathrm{E}+00 \mathrm{mGy}$ & 3.265E-01 mGy/day & $1.192 \mathrm{E}+02 \mathrm{mGy} /$ year \\
\hline Prostate & $6.602 \mathrm{E}+00 \mathrm{mGy}$ & 3.144E-01 mGy/day & 1.147E+02 mGy/year \\
\hline Testes & $6.714 \mathrm{E}+00 \mathrm{mGy}$ & 3.197E-01 mGy/day & 1.167E+02 mGy/year \\
\hline
\end{tabular}

\section{Figure 3.7: Example Effective Dose in OLTARIS}

to find the overall effective dose for the selected human model. The weighting factors used in OLTARIS are shown in Figure 3.8.

\begin{tabular}{|c|c|c|c|c|}
\hline Tissue Weights & 0.01 & 0.05 & 0.12 & 0.20 \\
\hline Tissue Types & Bone Surface & Bladder & Bone Marrow & Gonads \\
& Skin & Breast & Colon & \\
& & Liver & Lung & \\
& & Esophagus & Stomach & \\
& & Thyroid & & \\
& & Remainder & & \\
\hline
\end{tabular}

Figure 3.8: Tissue Weighting Factors[28]

Again, this is not an exhaustive list of every considered tissue or organ. It does not show the weighting of the adrenals, brain, small intestine, large intestine, kidneys, muscle, pancreas, spleen, thymus, uterus, as well as the heart, hippocampus, lens, and salivary glands. 


\subsubsection{Linear Energy Transfer (LET)}

Mapping the energy spectra of the radiation environment in LET is a useful means to understanding the biological components[28]. The LET response function is limited in the particles it considers by OLTARIS failing to account for anything other than light ion $(A<4)$. This study puts focus on both light and heavy ions so LET is not used in further analysis.

\subsection{Verification}

OLTARIS is a complex system written in FORTRAN with tens of thousands of lines of code, hundreds of files, and megabytes of data[28]. In addition, the tool is constantly updated to include new data and increasingly refined models. These updates can lead to a concern for lack of verification, however OLTARIS has several levels of verification steps in place to ensure accurate results.

The transport code HZETRN2005 with NUCFRG2 is a complicated system that requires in-depth analysis for verification. In order to validate results, the solutions determined through the codes can be compared to data collected in space applications as a proof of concept. Finding the deterministic solution through HZETRN allows for field mapping within the ISS through use of the Finite Element Method (FEM) using common geometries[31]. The light ion and heavy ion transport solution methodology is expressed in marching algorithms and have been analytically shown to be accurate to $O\left(h^{2}\right)[28]$.

All source code and data are stored in a version-control repository that all of the developers can access[28]. There are several types of checks performed to verify the program, module checks and functionality checks. Module checks ensure all modules of the code are preforming to standard. All developers are responsible to ensure 
accuracy of any new method applied in an OLTARIS update. This check is then stored in the repository for access. Functional tests are performed to verify interactions between modules to ensure the results generated are consistent. These functional tests are automatically rerun on a periodic basis to ensure that none of the additions break the system. In the case of a discrepancy, the developer is immediately notified to fix the problem. In addition, OLTARIS provides a change log to show the users when changes are made[28]. 
Chapter 4

\section{ORION CREW MODULE}

The Orion spacecraft is the baseline test specimen used for the analysis in this project. The intent is to show the beneficial effects of utilizing the required on-board water supply as additional radiation shielding. The Orion spacecraft was selected as it is on the forefront of human space exploration beyond the Van Allen Belts.

Orion is the flagship of the United States next generation of spacecraft, and the first U.S. human-rated spacecraft since the shuttle program. Orion consists of the Spacecraft Adapter, Supply Module, Crew Module, and Launch Abort System. The vehicle can be seen in Figure 4.1. From left to right is the adapter connected to the supply module, the crew module, and the launch abort system.
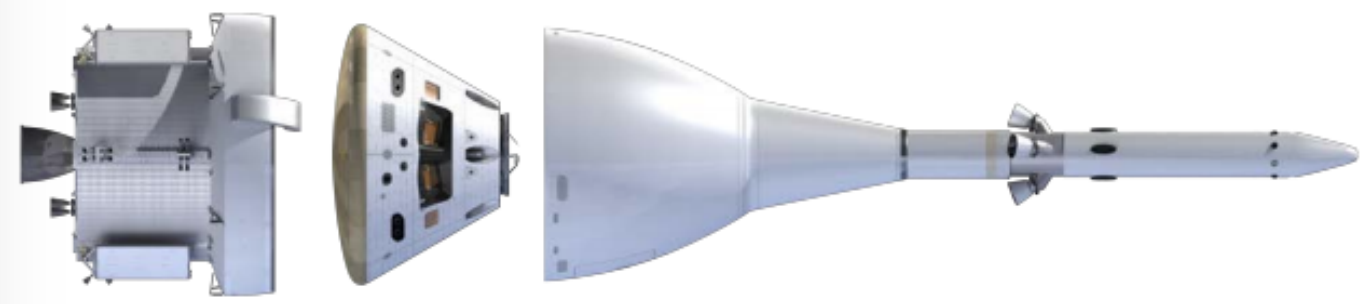

Figure 4.1: Orion Spacecraft[18]

The launch abort system enables the Orion spacecraft to eject away from the launch vehicle in the case of an emergency. It has the capability to respond within milliseconds to separate the astronauts from the launch vehicle.

In terms of radiation shielding, the most vital portion of the vehicle is the crew module, where the astronauts reside for the duration of the mission. The crew module design is inspired by the Apollo missions. This can be seen by the same shape utilized, the conical frustum. Several techniques are utilized by the Orion designers to mitigate 
the effects of the radiation environment including protection from several materials, coatings, and radiation protection plans. As the Orion capsule continues to be tested, the radiation shielding is updated. The specific updated information is not readily available to the public. The exact material composition throughout the vehicle profile is not public information and can not be modeled in this paper.

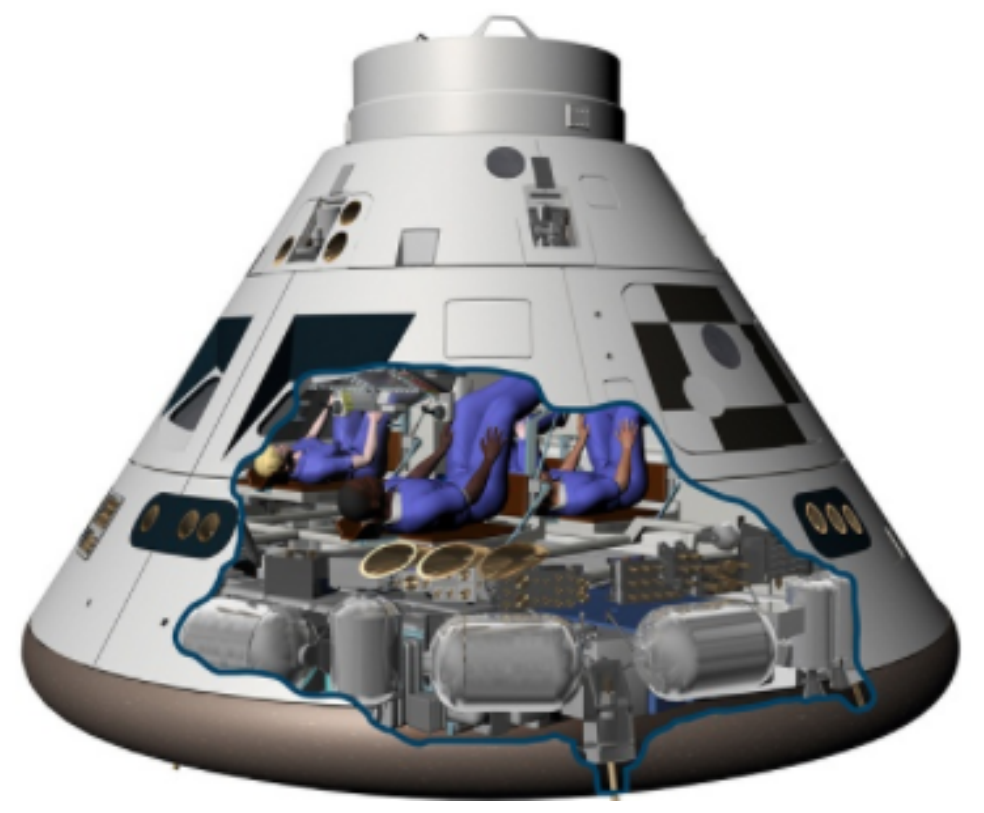

Figure 4.2: Orion Crew Module[13]

The supply module is responsible for supporting the crew for the duration of the mission until reentry into Earth's atmosphere. It holds water, oxygen, and nitrogen for the crew as well as thermal control, and power storage. The supply module is fit with four water tanks that are capable of supporting up to four astronauts for 21 days of flight. For longer duration flights Orion is designed to be able to support a crew for 210 days of additional quiescent mode in which crews will be required to switch to a deep space habitat module[18]. NASA has not yet locked in a final design for the deep space module, however they are currently looking into an inflatable option to save on volume during launch. In this scenario, the supply module would still contain the water for the mission as the crew module remains dormant. 
The remainder of this paper will be focused on the Crew Module portion of the spacecraft. In particular, taking the water of the supply module and applying it to the shield of the crew module.

\subsubsection{Limitations}

There is a limited amount of information for Orion that is available to the public. There was a test flight without a crew in 2013 to determine radiation values in flight. This information could be useful to verify results, however it is unavailable to the public. Due to the lack of information from Orion and the limitations through OLTARIS, the shielding makeup used in the test models has to be a simplified version of the Orion Crew Module. In addition, precise specs of each vehicle are not offered. The areal density of the vehicle shielding is not readily available, and has to be derived from the data that is presented. Without this exact information, uncertainty is introduced to the results that strays from the actual radiation exposure within the Orion capsule. Unfortunately, verification can't be done with the results in the 2013 test flight to determine the magnitude of this error. Despite this, the trends produced will still reveal valuable information to show the beneficial effects water contributes to the radiation shielding.

\subsection{Dimensions and Capabilities}

Orion has not been flown by humans yet but there is a goal to launch by 2022 on the newest NASA rocket, the Space Launch System (SLS). The current Orion planned flight is designed to house four astronauts for 21 days. The required water for this mission is $240 \mathrm{~kg}$ of water which would be held in tanks in the supply module[18]. This paper will propose moving that water from the supply module into the Crew Module as a permanent shielding fixture. 
Per the most recent report from NASA, the Crew Module has a maximum diameter of $5.02 \mathrm{~m}$ standing $3.3 \mathrm{~m}$ tall[18]. The pressurized internal volume is $19.56 \mathrm{~m}^{3}$ with $8.95 \mathrm{~m}^{3}$ of habitable volume for the crew. The most prominent material within the crew module is aluminum lithium.

\subsubsection{Emergency Radiation Protection Plan}

This project will show the difference of using water as a permanent shielding fixture as opposed to the current NASA plan. The following will detail the emergency radiation plan NASA has in place in the event of a major solar storm. In this case, a portion

of the on-board water would be allocated to shielding astronauts. For SPE testing, it is important to include this portion of water to the baseline test to show the benefit of using water as permanent shielding.

In the event of an impending solar event, Orion currently has a radiation mitigation strategy to best protect the crew. If the crew is alerted prior to an event or alerted by on-board sensors that an event has already begun to penetrate shielding, they will begin emergency procedures to add protection[7]. This consists of building a shelter at the center of the crew module out of all the supplies including all accessible food and water[7]. Enough food and water will be stored for the astronauts to remain in the bunker for over a day. The process is shown in Figure 4.3 with the astronauts covering themselves with equipment to provide maximum shielding thickness. This plan requires quick response from the crew to get to safety as soon as possible. This means that the astronauts only use material easily accessible to build the emergency bunker. Although some water will be used in the emergency bunker, only the water that is in the crew module helps the astronauts. The remainder of the water is left in the supply module water tanks.

Not all $240 \mathrm{~kg}$ of water can or will be utilized in the event NASA needs to execute 


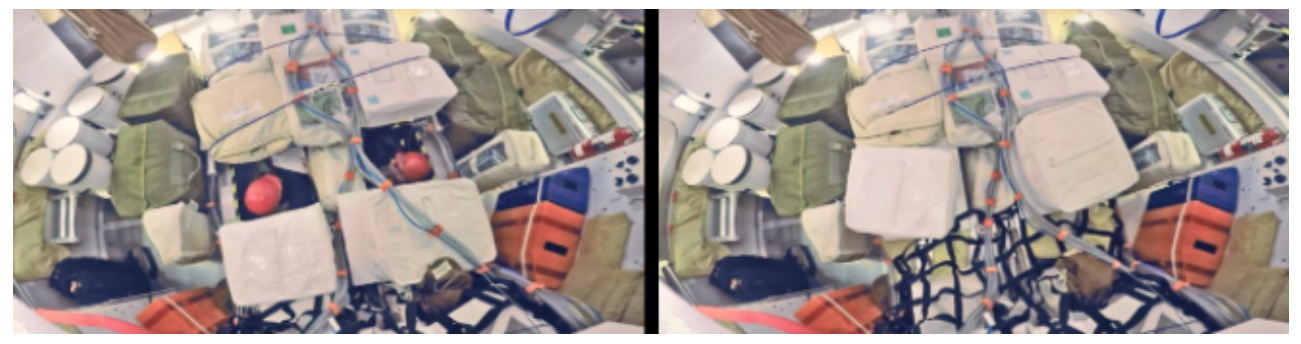

Figure 4.3: Emergency Radiation Protection Plan[7]

the emergency radiation plan. The exact thickness profile of the water used for the emergency plan is not a readily available number for public access. However, the scope of this project intends to show the difference between the current NASA plan and the plan to utilize water as a permanent shielding fixture. In order to effectively do this, it is important to include the water that would be utilized in the current emergency radiation protection plan. This requires some level of estimation to determine the thickness of water that protects the astronauts. The assumptions made are stated below.

It is known that the emergency bunker will consist of enough water to sustain four astronauts for periods of time longer than a day. Astronauts are limited to three gallons of water a day aboard the ISS, which would be approximately 88 Liters for two days[16]. With margin for contingency, it can be liberally assumed that the maximum amount of water that would be accessible to the astronauts would be 100 Liters. Figure 4.4 provides the internal volume required for an astronaut in radiation shelter. This area is multiplied by the number of astronauts to determine the radiation bunker size for an Orion scenario. The water would be stored above the astronauts in the proposed plan in the provided water storage bags.

Utilizing the volume required for four astronauts and the volume of water accessible by the astronauts, the thickness of water in the emergency bunker can be determined. It was assumed for this calculation, the area of coverage would be over- 


\begin{tabular}{c|c|c|c|c}
$\begin{array}{c}\text { Figures of Human Body } \\
\text { Postures and Volumes }\end{array}$ & $\begin{array}{c}\text { Applicable } \\
\text { Functions }\end{array}$ & \multicolumn{2}{|c|}{ Dimensions (m) } & $\begin{array}{c}\text { Volume } \\
\left(\mathbf{m}^{3}\right)\end{array}$ \\
\hline & & $\mathrm{H}$ & 2.06 & \\
\cline { 1 - 2 } & \multirow{2}{*}{$\begin{array}{c}\text { Eating, sleeping, } \\
\text { hand washing, } \\
\text { personal office, } \\
\text { radiation shelter, } \\
\text { conference }\end{array}$} & $\mathrm{L}$ & 1.06 & \multirow{2}{*}{$\mathbf{2 . 6 9}$} \\
& & $\mathrm{W}$ & 1.23 &
\end{tabular}

Figure 4.4: Emergency Radiation Protection Plan[14]

head of all the astronauts. This limits allows the thickness to be determined. This volume of water would cover approximately $25 \%$ of the astronauts at $2.0 \mathrm{~cm}$ of thickness.

It is important to note these assumptions for the emergency radiation bunker situation when investigating the results. There is a strong possibility the thickness of water and percent coverage are over-estimates for the system. The thickness, size, and number of water bags is not provided by NASA which requires an additional assumption to determine the dimensions of the shield.

It is also important to note that in the permanent water shield scenario, it is assumed that there will still be an emergency radiation protection plan in place to protect the astronauts with food and supplies. This will isolate the effects of water specifically in the tests, as the other components will be omitted from all test scenarios. 
Chapter 5

\section{OLTARIS TOOL}

As discussed earlier, OLTARIS requires a specific thickness distribution XML file to be inputted in order to run. This project utilizes a MATLAB code initially designed for PPOD CubeSats to create the necessary thickness distributions[10]. This tool had to be refined in order to create new geometries as well as have the ability to import multiple materials. The new improved tool, the Vehicle Thickness Distribution Tool, was developed as a major portion of this project.

The tool specifically reads .stl CAD files, which is the Standard Triangle Language. The CAD model is converted to MATLAB matrices using triangles and vertices in a position space. Units and material types are not carried in .stl format, so the user must input this in the code.

The ray file downloaded from OLTARIS is imported into the code and used to ray-trace the geometry within MATLAB. The rays protrude out from the target point in all directions to determine the thickness distribution of the shielding. This is done in the code through use of the rays intersecting the faces of the triangles and using the distance formula along with a unit conversion. The tool then uses the data to create a properly formatted XML file to be inputted into OLTARIS. This process will be detailed thoroughly in the following sections.

\subsection{Tool Functionality}

The tool uses code downloaded from MATLAB Central, cad2mat.m, to convert the CAD into matrices in which the triangles from the .stl file are saved as vertices, faces, and color. The color is strictly aesthetics and provide no valuable results for the user. 
Figure 5.1 shows the plotted vertices for a sphere test case. Units are not carried over from the CAD file, and the origin is not always carried over correctly through the file export. It is crucial to export the CAD with the origin defined in the center of the specimen. To prevent this from causing issues, the tool plots the CAD once it is converted into MATLAB. Through visual inspection, the user should ensure the test vehicle is centered about the origin.

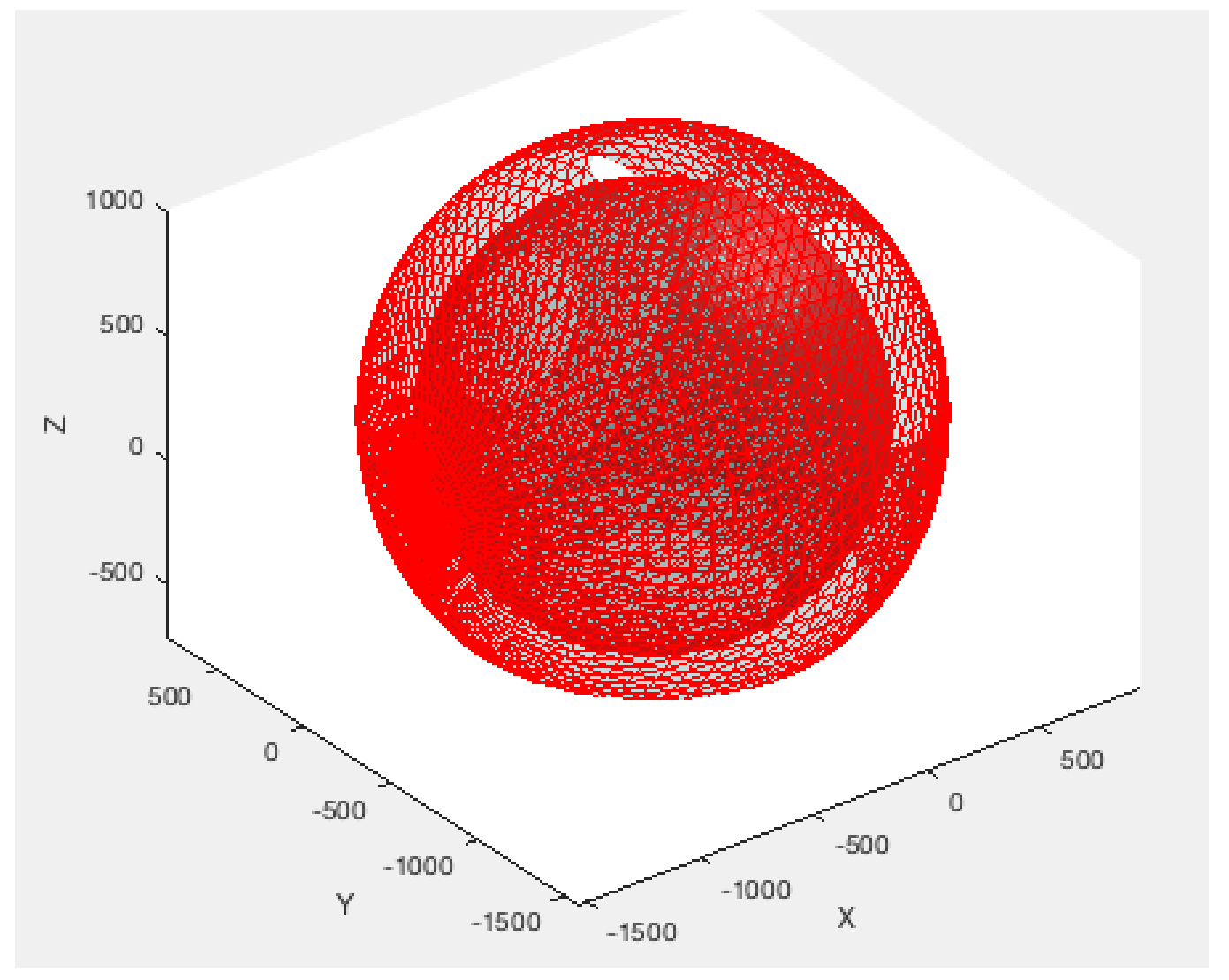

Figure 5.1: CAD model of sphere imported to MATLAB

In order to create the thickness distribution, the tool must then have the capability to ray trace the sphere shown in Figure 5.1.

The tool reads in both the vehicle model and the ray file. A target point is determined by the user as the origin for all the rays. For each individual ray, the code loops through and determines intersection points between the ray and any triangle 
face. Every triangle face is considered for each ray which leads to the increased computational times seen in Table 5.1. Once all the intersecting faces are determined, the triangle vertices are used to determine if the ray lies within the borders of the face. After this step, the code has identified each face the ray has intersected with. The method implemented utilizes the parametric plane equation (Equation 5.1) and the intersection point algorithm[10].

$$
V(s, t)=V_{0}+s\left(V_{0}-V_{1}\right)+t\left(V_{2}-V_{0}\right)=V_{0}+s \mathbf{u}+t \mathbf{v}
$$

$V_{0}, V_{1}$, and $V_{2}$ represent the three vertices, and the vectors are $\mathbf{u}$ and $\mathbf{v}[10]$. This is represented in Figure 5.2.

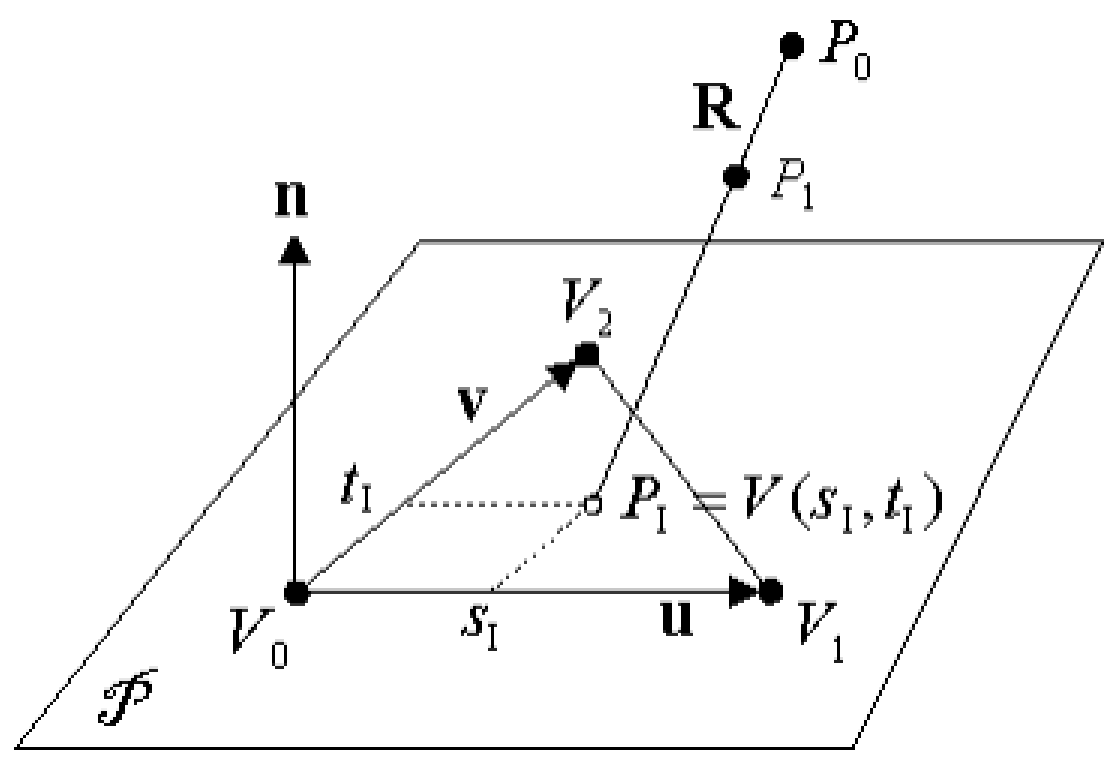

Figure 5.2: Intersection Point Algorithm[1]

The real numbers $s$ and $t$ are determined through a series of dot products detailed in Equation 5.2 and Equation 5.3 respectively[10].

$$
s=\frac{(\mathbf{u} \cdot \mathbf{v})(\mathbf{w} \cdot \mathbf{v})-(\mathbf{v} \cdot \mathbf{v})(\mathbf{w} \cdot \mathbf{u})}{(\mathbf{u} \cdot \mathbf{v})^{2}-(\mathbf{u} \cdot \mathbf{u})(\mathbf{v} \cdot \mathbf{v})}
$$




$$
t=\frac{(\mathbf{u} \cdot \mathbf{v})(\mathbf{w} \cdot \mathbf{u})-(\mathbf{u} \cdot \mathbf{u})(\mathbf{w} \cdot \mathbf{v})}{(\mathbf{u} \cdot \mathbf{v})^{2}-(\mathbf{u} \cdot \mathbf{u})(\mathbf{v} \cdot \mathbf{v})}
$$

Once the intersection point is found for a ray and a triangle, three conditions must be checked: $s \geq 0, t \geq 0$, and $s+t \leq 1[10]$. If all conditions are satisfied, the intersection point is verified.

All intersection points are saved in a matrix for each ray, and the thicknesses are determined through the distance formula. Once all rays are computed, there is a complete thickness profile that can be exported to match the required OLTARIS XML file format. The process is depicted in Figure 5.3 up to the point where the thickness profile is saved in a large matrix.

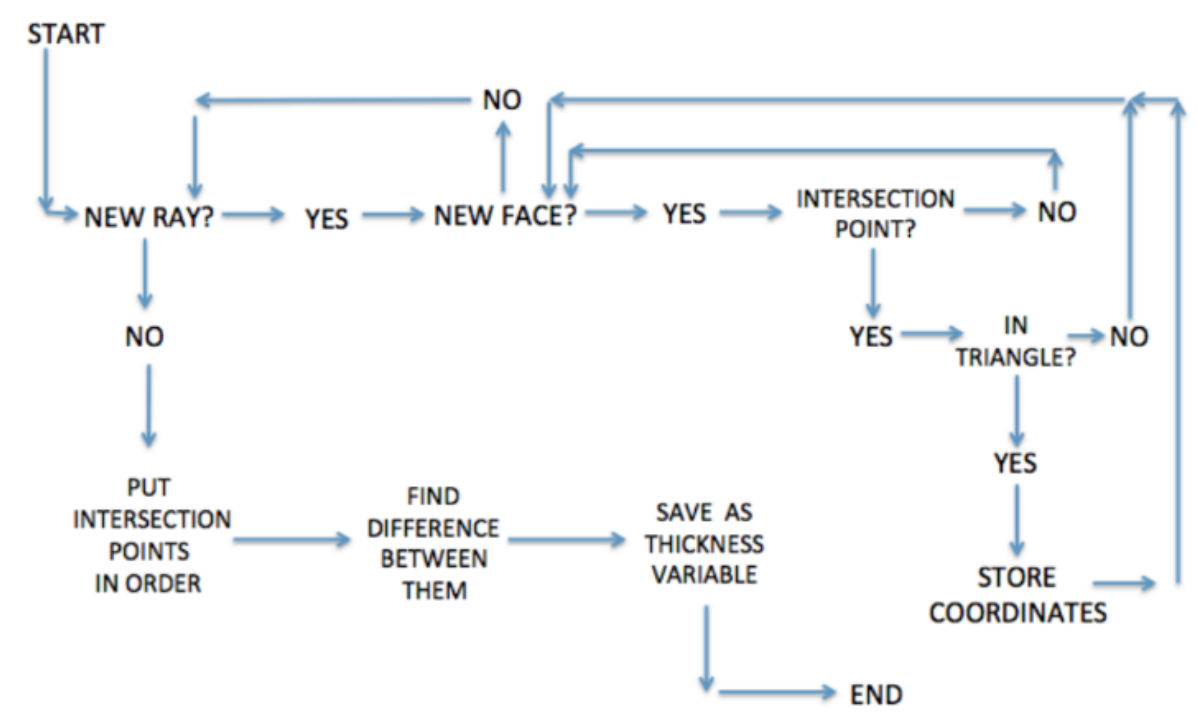

\section{Figure 5.3: MATLAB Vehicle Thickness Distribution Tool Flow Chart[10]}

The default ray distribution is 1002, which provides for higher fidelity results with smaller computational times. Only the geodesic ray distributions are compatible with the tool, which excludes 512, 968, and 10000 ray distributions. Table 5.1 shows the difference in ray distribution file by dosage and computation time compared to a preset sphere built into OLTARIS. This specific case is an Orion-sized test sphere 
in the GCR environment. The dimensions of the test sphere will be detailed in the section 5.4 .

Table 5.1: Ray Distribution Associated Variance

\begin{tabular}{lccc}
\hline Ray Distribution & $\begin{array}{c}\text { Computation Time } \\
(\mathrm{sec})\end{array}$ & $\begin{array}{c}\text { Dose Equivalent } \\
(\mathrm{mSv} / \text { year })\end{array}$ & $\begin{array}{c}\text { Percent Variance } \\
(\%)\end{array}$ \\
\hline 42 & 24 & 21.72 & 5.66 \\
492 & 266 & 20.74 & 1.21 \\
$\mathbf{1 0 0 2}$ & $\mathbf{5 3 3}$ & $\mathbf{2 0 . 7 1}$ & 1.06 \\
4002 & 2555 & 20.57 & 0.389 \\
9002 & 7186 & 20.50 & 0.049 \\
Preset Sphere & - & 20.49 & - \\
\hline
\end{tabular}

The computational times increase drastically as the ray files become more robust, leading to an acceptable variance in the results. As seen in Table 5.1, the 42 ray distribution is considerably less accurate than the other cases. It could be argued that the 492 ray distribution is more optimal when it comes to time efficiency, however the 1002 ray distribution was selected as the baseline for all tests to narrow any uncertainty.

\subsection{Revisions}

There were several restrictions initially with the code, which led to a major overhaul to generalize the tool and make it applicable for a variety of uses. Due to the nature of the code utilizing faces of triangles, the code is limited in geometries that can be applied. For example, the corners of a CubeSat read a thickness of zero as the ray intersects no faces of a triangle. It is particularly dependent on the method of which the geometry was created in the CAD software due to the necessity of having the triangle faces be perpendicular to the vector of the ray. If not, the thickness will be 
incorrectly read as zero. Previously, the tool was only utilized for CubeSats, which allowed the tool to be tuned for the specific application.

The tool has been modified to handle corners and sharp geometries without relying on hard-coded inputs. The problems arise when an uneven number of faces or zero faces are intersected by the ray. This occurs with sharp, irregular geometries. Through the process used in this tool, it is much easier to ray trace a sphere than a cube. To counter this effect in the tool, rays are analyzed around the point of interest. If a ray determines an uneven or zero number of interesting faces, it is marked in the tool. The unmarked rays in the direct vicinity of the marked rays are taken and the thickness values are averaged to determine the probable thickness of the marked ray in question. Figure 5.4 shows a marked ray surrounded by unmarked rays with defined thickness distributions. In this case, all eight surrounding rays data is averaged to determine the probable thickness distribution of the center unmarked ray. Before the unmarked ray is redefined with the probable thickness values, the average is compared to the eight rays values. If one or more of the eight rays strays from within $10 \%$ of the probable value, they are removed and a new probable value is used for the unmarked ray. If over four rays are beyond the designated $10 \%$ variance value, the original probable thickness value is used.

Another possibility must be considered where multiple unmarked rays occur within the same vicinity, which is often the case for corners and sharp edges. The process follows the same pattern as before with an increased direct vicinity as shown in Figure 5.5. In this case the number of reference rays increases to ten, and the same process takes place to determine the thickness distribution of the unmarked rays. Both unmarked rays would then take on the probable thickness values determined.

The process is run through a loop once all of the thickness values have been determined. The code takes all unmarked rays and locates the rays in the direct 


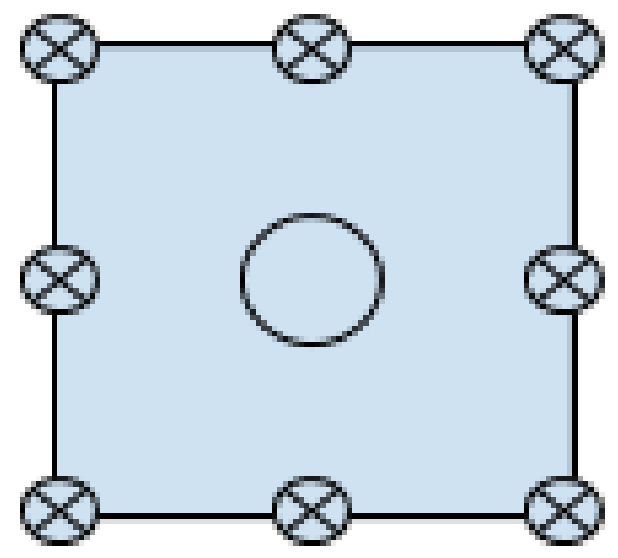

Figure 5.4: Undefined Ray Thickness Process

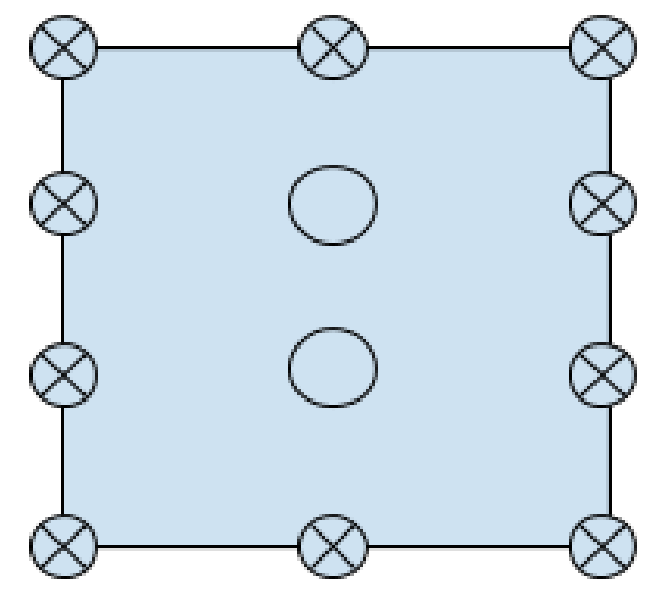

Figure 5.5: Multiple Undefined Rays Process

vicinity. If all the rays in that zone have values, the thickness profile is determined. After those unmarked rays are solved, the tool addresses groupings of unmarked rays from smallest to largest. The process described before calls to expand the vicinity of interest if an unmarked ray lies on the edge. If the vicinity is expanded and an unmarked ray still lies on the edge, the vicinity is frozen and the probable thickness is calculated. This process allows for a complete vehicle thickness distribution to be created for more complicated geometries. It relies on estimation and induces error to 
the system, so each unmarked ray is noted and displayed after the final calculation to give the user a physical number on the amount of estimations made.

Aside from issues that arise with the intersection method, the original CubeSat code was written to support only one material. It is imperative to be able to determine the different materials and the associated thickness of all materials for more complicated cases. This provides a hurdle, as the CAD file does not indicate, or differentiate between, different materials. Initially, there was hope that the color data imported within the .stl CAD file could be used to differentiate materials. This led to more issues as it became CAD specific, and requires CAD models to be a specific format. This goes against the desire to generalize the code for multiple uses. In order to tackle this issue, the code was altered to include material inputs. The user of the tool can specify new materials and the desired thickness of this material. For the case of the Orion spacecraft, the CAD is converted to triangles and vertices and the thickness distribution is created as described above. As the script begins to create the XML formatted file, it adds a second line to each ray with the specified thickness of the desired material. It is essentially a workaround to ray tracing the new material. The new procedure was tested against a preset OLTARIS sphere to determine the validity of the method.

The setup for this case is an Orion-sized test sphere composed of aluminum lithium with an added thickness of water in the GCR environment at $1 \mathrm{AU}$ of free space. Again, the dimensions of the test sphere will be determined in section 5.4. The sphere created from the Vehicle Thickness Distribution Tool utilized the 1002 ray file from OLTARIS. This was tested and compared to OLTARIS results to validate the method as shown in Table 5.2.

Table 5.1 gives an indication that the 1002 ray distribution file has an associated variance from the preset OLTARIS sphere. The percent variance in Table 5.2 is 
Table 5.2: New Material Implementation

\begin{tabular}{lcc}
\hline Case & Dose, $\mathrm{mGy} /$ year & $\%$ Variance \\
\hline OLTARIS defined Sphere & 374.6 & - \\
CAD Sphere Modified & 371.3 & 0.9 \\
\hline
\end{tabular}

smaller than the associated ray uncertainty. This gives a strong indication that the methodology utilized to create the thickness distribution with multiple materials is valid. Through analysis of the XML file, it becomes apparent that the methodology produces an accurate vehicle thickness distribution. The thickness of the new material is uniform throughout the distribution, rather than fluctuating around the mean as it does with the ray-tracing method. This caters closer to the preset sphere with uniform thickness throughout. As the number of rays are increased, there is less fluctuation about the mean when ray-tracing which leads to higher fidelity results. A similar effect of higher fidelity results is seen with the new material implementation. This proves to validate the methodology used in the Vehicle Thickness Distribution Tool.

In addition to adding new materials, a component was added to the tool to add a new material to only a percentage of the vehicle. This allows the user to customize the vehicle and implement aspects that are not uniform around the exterior, such as a heat shield. In this application, this function specifically enables the creation of a thickness distribution of the Orion Crew Module in the emergency radiation bunker situation, where water only protects $25 \%$ of the astronauts. The code allows the user to input a percent coverage for a new material. Only that percent of rays is then populated in the thickness distribution that is created. This is shown in Figure 5.6, as only a portion of the spacecraft would be covered in a new material. In this figure, the orange represents the rays that would include water, and the blue represents rays that would remain without it. This allows for testing the emergency radiation 
protection plan proposed by NASA.

Figure 5.6 shows the tool implements coverage on $25 \%$ of the rays in the same vicinity. The phantom body models can be oriented specifically within the vehicle to ensure the water coverage is directly overhead of the astronauts, which is desirable for testing configuration. Although SPE is directional, OLTARIS models the particle transport as isotropic, and vehicle orientation is therefore irrelevant. It is ideal to protect against SPE directionally, however, the fast nature of the events make it difficult to plan accordingly. The current emergency protection plan for the crew module does not directionally protect for SPE. Rather, it consists of sheltering at the center of the spacecraft with supplies spread evenly around the astronauts. For these reasons, the directionality of the water shielding with respect to the impending SPE is not critical for testing.

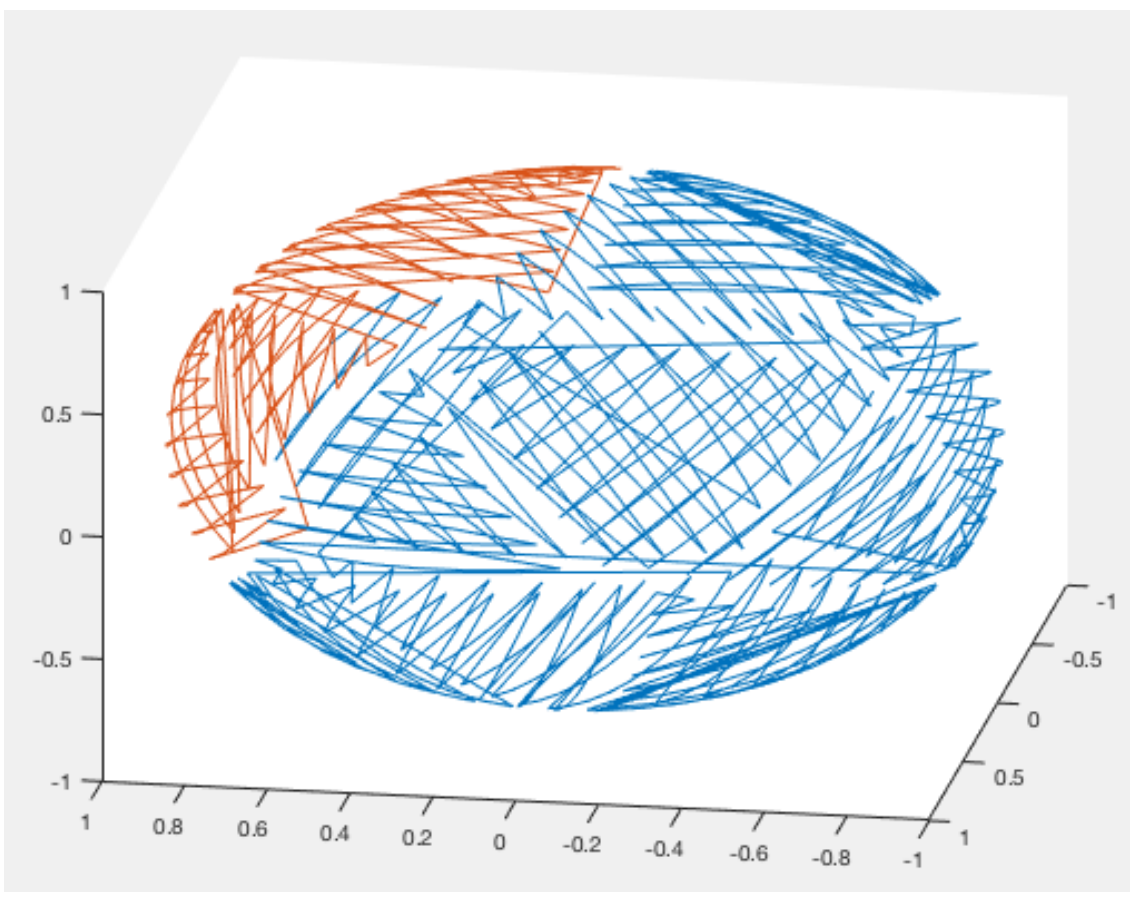

Figure 5.6: Visual of $25 \%$ of Rays Covered in New Material 


\subsection{Limitations}

In order to determine if the CAD geometry is sufficient to be ray-traced, it is plotted for the user to inspect. As seen in Figure 5.1, the sphere has the faces of the triangle perpendicular to a ray that protrudes from the center of the shape. Conversely, The Orion Crew Module CAD was created differently and the triangles are incompatible with the ray-tracing tool. This is visible in Figure 5.7. The problem lies with the methodology of ray tracing. As the rays emanate out from the target point they need to intersect the faces of the triangles to create an intersection point to determine a thickness. In this case for Orion, the triangle face plane is parallel to the rays. This leads to very few intersections and a majority of the measured thickness values to be zero.

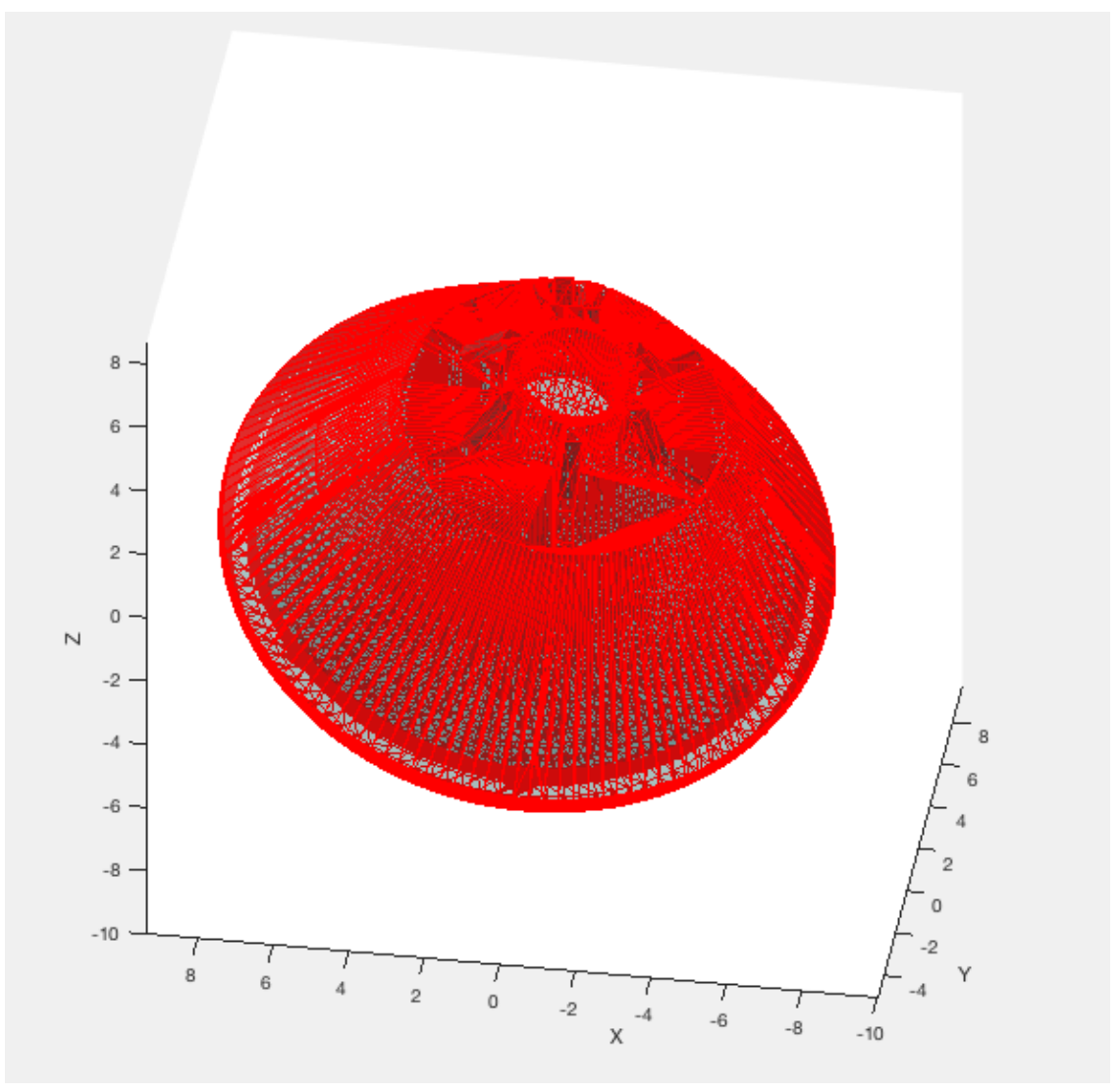

Figure 5.7: Orion CAD processed by MATLAB tool 
To continue with testing there are several directions to take. One option would be to create a new ray-tracing tool that can read the specific CAD. A new ray-tracing tool would be time costly, and is unnecessary to solving the problem. Rather, the second option is to simplify the shape of the Orion capsule to a sphere of equal internal volume and equal wall thickness. The OLTARIS computational method relies heavily on the thickness and material type between the target point and the radiation environment. So long as the Orion capsule has a relatively uniform thickness throughout the vehicle profile, simplifying the shape to a sphere is harmless. The scope of the project is to determine the benefits from utilizing water as radiation shielding. This is unencumbered by the spherical assumption, and valuable results are still produced.

\subsection{Orion Crew Module Simplifications}

Testing the crew module is not a trivial task and requires simplifications in order to produce results within OLTARIS. In order to test the crew module, a thickness distribution file must be produced to input into OLTARIS. As mentioned in previous sections, the conical frustum shape of the crew module has to be simplified into a sphere. In order to do this, the thickness of the shield for the sphere must be determined from the frustum.

The volume of the crew module is described in Equation 5.6. Using this equation and the known interior and exterior volume, the dimensions of shield can be determined through the limited knowledge provided in NASA data sheets. As described previously, the Crew Module has a maximum diameter of $5.02 \mathrm{~m}$ standing $3.3 m$ tall[18]. Equation 5.6 is then used to find the external volume of the module and then the thickness. The angle, $\theta$, of a conical frustum by definition is $57.7^{\circ}$, where $\theta$ is defined in Equation 5.4: 


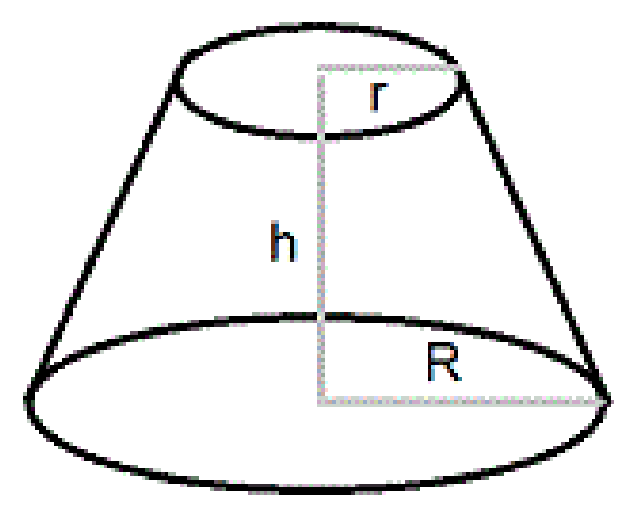

Figure 5.8: Conical Frustum[2]

$$
\tan (\theta)=\frac{h}{x}
$$

where $\mathrm{x}$ is described in Equation 5.5.

$$
\begin{gathered}
R-x=r \\
V=\frac{\pi h}{3}\left(R^{2}+R r+r^{2}\right)
\end{gathered}
$$

With this, the exterior volume of the Orion capsule is determined to be $26.069 \mathrm{~m}$, and the thickness of the spacecraft is approximately $16.79 \mathrm{~cm}$. With a known interior pressurized volume of $19.56 \mathrm{~m}^{3}$, an Orion-sized sphere can be determined using the same volume and thickness values of the Orion capsule. This spherical model has an external radius of $1.8394 \mathrm{~m}$ and an internal radius of $1.6715 \mathrm{~m}$.

The default input within OLTARIS, as defined before, is areal density in $\mathrm{g} / \mathrm{cm}^{2}$. Using aluminum lithium as the sole material in the crew module, the areal density of the Orion Crew Module is $45.33 \mathrm{~g} / \mathrm{cm}^{2}$. This is the main shielding component utilized by NASA in the Orion project. Due to the limited data, there are simplifications and 
an assumption that there is no empty space between the pressurized volume and the external volume. This potentially leads to a higher estimation for the areal density of shielding than the Orion Crew Module has in actuality. This affects the results in terms of proximity to what astronauts would be exposed to physically on-board the Orion. However, there are many unknown factors unavailable to the public that make it impossible to model a system that would accurately predict the radiation values in an Orion mission. On the contrary, this will not compromise the data in terms of the effect water has as a permanent component of the radiation shielding.

Now, the thickness of the water shield can be determined using the $240 \mathrm{~kg}$ of allotted mass. This is equivalent to 240 Liters of water, which leads to a thickness of $0.8 \mathrm{~cm}$ around the entire interior shielding of the spacecraft.

The simplified Orion sphere is modeled in SolidWorks as seen in Figure 5.9. The water is not implemented within the CAD model, but rather, will be introduced into the thickness distribution through the tool developed for the project as describe earlier. It is an easier process to modify the file within MATLAB to include the water.

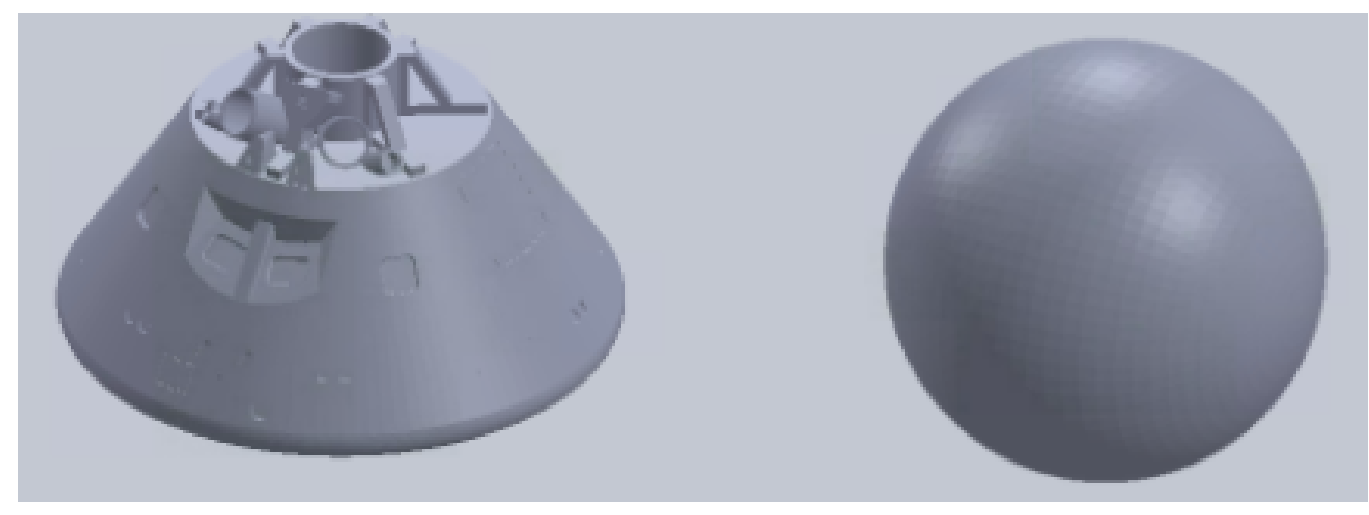

Figure 5.9: CAD model of simplified Orion sphere. 


\section{Chapter 6}

\section{RADIATION TESTING SETUP}

Using OLTARIS defined environments, the Orion test sphere can be tested within the GCR environment as well as the SPE environment to show the effects of the additional water shielding.

In addition to the simplifications detailed earlier, it is assumed there is only one astronaut on-board the Orion for the duration of the mission. To isolate the effects of the water shield, the astronaut remains in the same position and orientation at the center of the crew module for the duration of the mission. This is not a realistic assumption, however, it serves as an average position for the astronaut throughout the duration of the mission. Orientation differences are negligible within a test sphere, and in the tight confinement of the crew module, a majority of the time will be spent near the center. Adding to this, the livable interior volume is only $8.95 \mathrm{~m}^{3}$, which is less than half of the pressurized interior volume. This keeps the astronauts near the center of the test sphere. Adding additional astronauts would require advanced techniques and programs not available to the public, and is unnecessary to determine the effects water has in radiation shielding.

The simplifications made previously allow many of the tests to be done completely within OLTARIS besides the emergency bunker plan. A simplified Orion sphere can be created inside the OLTARIS website and eliminates the need for the raytracing tool developed for the project. However, the ray-tracing tool is still needed to implement the test case for the emergency radiation bunker with a percent coverage. Utilizing the developed tool to determine effects for the other cases is unnecessary and adds uncertainty to the results, so the tool is only used for the emergency bunker situation. 
For every test within OLTARIS, the environment specified will be 1 AU of free space to replicate the effects of deep space radiation unencumbered by the protection from the Van Allen belts. This is an accurate model for deep space missions, allowing for a general analysis for a variety of missions. Current NASA plans are focused on shorter-duration missions to the Moon to test the Orion capsule with human passengers for the first time. NASA also plans to use Orion for future missions well beyond the Moon after several successful flights within the Earth's sphere of gravitational influence. Mars missions transit from approximately 1 to 1.5 AU from the sun. Due to lack of data, OLTARIS does not model an environment other than 1 AU despite there being a difference in location within the heliosphere. To provide analysis for multiple missions, 1 AU free space analysis is the preferred method.

This project will focus on dosages from historical SPE that span the duration of the event, as well as GCR models. The GCR models will yield results for a shorterduration Moon mission, but will also output the radiation dosages per year. This is a particularly beneficial output as the estimated time of round trip travel for a Mars mission is approximately a 12-month venture. This data can provide information for both the current planned moon missions as well as for future long-duration missions.

The proposed permanent water shield from this study consists of $45.33 \mathrm{~g} / \mathrm{cm}^{2}$ of Aluminum-lithium and $0.8 \mathrm{~g} / \mathrm{cm}^{2}$ of water. It is assumed the $0.8 \mathrm{~g} / \mathrm{cm}^{2}$ areal density of water remains constant throughout the duration of the mission. For long-duration missions, there would be a water reclamation system that would be incorporated in the system. However, this is not considered in the scope of this study, and it is assumed the thickness of water would remain unchanged. This is not far from actuality if the water recycling system takes place within the shield. 


\subsection{SPE Environment}

There are two cases considered for the SPE tests. The first case is the proposed permanent water shield of $0.8 \mathrm{~cm}$ of protection. The second is NASA's current plan of utilizing an emergency radiation bunker consisting of $2.0 \mathrm{~cm}$ of water shielding with $25 \%$ coverage of the astronaut to model the current emergency radiation contingency plan.

The test in Table 6.1 consists of the baseline Orion Crew Module sphere with no water shielding exposed to various events at $1 \mathrm{AU}$ of free space. Each event within OLTARIS is tested to show the difference in magnitude of each storm, and to determine the worst-case scenario for testing. The first event used for analysis is the Carrington event of 1859, which is considered the most powerful SPE in recent history.

The event of October 1989 was one of several powerful solar storms that year considered the most powerful storm ever measured. It is shown in Table 6.1 as the most powerful solar storm of the year, and the most powerful storm included in OLTARIS from the $20^{\text {th }}$ century. The comparison is done for both the male and female models (CAM and CAF).

OLTARIS has the built-in ability to determine effective doses for human models in simple geometries. As mentioned earlier, a majority of the calculations can be accomplished without the developed tool. The developed tool enables the incorporation

of rotated human models and variable target points within the vehicle distribution, as well as more complicated vehicle models. For the scope of this project, the phantom body will remain centered in the spacecraft in the standard position. 


\section{Table 6.1: Solar Particle Event Comparisons}

\begin{tabular}{lccc} 
Solar Particle Event & $\begin{array}{c}\text { Dose } \\
(\mathrm{mGy})\end{array}$ & $\begin{array}{c}\text { Effective Dose (CAM) } \\
(\mathrm{mSv})\end{array}$ & $\begin{array}{c}\text { Effective Dose (CAF) } \\
(\mathrm{mSv})\end{array}$ \\
\hline Carrington 1859 & $\mathbf{4 6 . 8 0}$ & $\mathbf{2 9 2 . 0}$ & $\mathbf{2 9 3 . 3}$ \\
February 1956 Webber & 10.92 & 25.98 & 26.15 \\
February 1956 LARC & 15.51 & 27.73 & 27.91 \\
November 1960 & 31.87 & 82.29 & 82.70 \\
August 1972 King & 9.82 & 24.35 & 24.67 \\
August 1972 LaRC & 10.05 & 23.47 & 23.75 \\
September 1989 & 29.28 & 56.79 & 57.13 \\
October 1989 & $\mathbf{3 3 . 3 6}$ & $\mathbf{9 3 . 9 6}$ & $\mathbf{9 4 . 4 4}$ \\
\hline
\end{tabular}

\subsection{GCR Environment}

As discussed with the SPE testing, it is desired to analyze the worst case scenario for the GCR environment. Through solar data observed over the last several decades, it is determined the worst-case scenario occurred during the 1997 solar minimum. As shown in Table 6.2, the 1997 solar minimum produced the largest flux of GCR particles.

Table 6.2: Galactic Cosmic Ray Comparisons

\begin{tabular}{lc} 
Historical Minimum & Dose Equivalent, mSv/year \\
\hline 1965 & 358.9 \\
1977 & 368.4 \\
1987 & 354.7 \\
$\mathbf{1 9 9 7}$ & $\mathbf{3 7 3 . 2}$ \\
2010 & 370.4 \\
\hline
\end{tabular}

There are two cases of importance to analyze in the 1997 solar minimum GCR 
environment. The first case is the proposed permanent water shield on the Orion Crew Module with the $0.8 \mathrm{~cm}$ of water. The second is the Orion Crew Module, with no additional protection.

\subsection{Uncertainty}

There are several required assumptions and simplifications made in this model, which adds a level of uncertainty to the results. In addition, the deep space radiation environment models used are estimating tools with no known associated errors[31]. Currently, there is an extremely limited amount of data with no methods of verification, leading to imperfect results. In addition, there is an uncertainty that is produced in the self-developed tool. This was introduced in the previous section when verifying the tool with OLTARIS pre-loaded spheres. This uncertainty is determined for each case and reported for all results that utilize the tool.

It is known that the HZETRN transport code, along with all other current GCR codes, don't properly treat the three dimensional nature of fragmentations produced from HZE particles, which has some associated uncertainty with biological risk at critical body organs[31]. The assumptions made within the transport code were detailed more thoroughly in chapter 3 , however, this is the best model available currently to determine radiation dosage in space.

An important point to note is all cases are run with the same imperfect model. Currently, it is the best accessible option and is commonly used in industry despite the inherent shortcomings. Along with the simplifications made to the model in order to produce results within OLTARIS, these uncertainties will not compromise the trends produced showing the effects of water as a radiation shield. The uncertainties would only contribute to uncertainty for radiation levels seen in flight. Without radiation data from previous test flights, this verification is beyond the scope of this project. 
Despite the uncertainties described above, they affect both cases equally in testing. This enables comparisons to be made between the test cases leading to valid results, as well as enabling viable conclusions to be made from the results. 


\section{Chapter 7}

\section{RESULTS}

Analysis was completed for phantom bodies within the ORION test sphere. This section will breakdown the OLTARIS output numbers for the Solar Particle Events as well as for the GCR environment. As expected, the water shield performs better for protecting against solar events than for GCR. Variable shielding thickness and multiple shielding configurations were considered in analysis to improve the performance

of water in the GCR environment. To reduce uncertainty in the results determined, OLTARIS pre-set spheres were utilized as much as possible. This creates more validity to results that have tight margins, which occurs in the GCR environment in particular. As described in earlier sections, OLTARIS has an unknown associated error. The only values reported in this section with uncertainties are the values that were created from the developed tool described in the previous section. All other values are affected by the same unknown uncertainty within OLTARIS, which enables comparisons to be made.

\subsection{SPE Analysis}

As detailed earlier, two historical solar events are considered as worst case scenarios for testing. The following subsections will break down the results for both events to determine the effects of utilizing on-board water as a permanent shielding fixture. In the event of a massive storm, the astronauts on-board the crew module would assemble the emergency bunker of food, water, and supplies. Since testing is analyzing the worst case scenario, the two cases must be the proposed permanent water shield, versus the current emergency bunker plan. 


\subsubsection{Effect of Water}

It is well documented water has a positive effect on radiation shielding, however, it is important to confirm the positive effects. Table 7.1 shows the benefit a permanent water shield has over the baseline vehicle with no additional water. This case is tested in the radiation environment of the Carrington event. Table 7.2 shows the same test for the October 1989 event. The positive effect of the water is expected, and shows the minimal thickness of water is still helpful.

Table 7.1: Carrington Event Radiation Exposure: Long-Term Effects

\begin{tabular}{lcc}
\hline Parameter & Permanent Shielding & Baseline Vehicle \\
\hline CAM Effective Dose Equivalent, mSv & 277.5 & 296.0 \\
CAF Effective Dose Equivalent, mSv & 278.7 & 296.8 \\
\hline
\end{tabular}

Table 7.2: October 1989 Event Radiation Exposure: Long-Term Effects

\begin{tabular}{lcc} 
Parameter & Permanent Shielding & Baseline Vehicle \\
\hline CAM Effective Dose Equivalent, mSv & 89.77 & 98.0 \\
CAF Effective Dose Equivalent, mSv & 90.14 & 98.3 \\
\hline
\end{tabular}

Clearly, water has a beneficial effect on the radiation shielding. The more coverage, the better results for astronauts on-board the spacecraft. It acts as a retardant to the particles ejected from the sun to mitigate the effects on the astronauts. It is wasteful to design a system where on-board water is not permanently utilized. As mentioned earlier, the effective dose equivalent tests are limited in the amount of materials able to be tested. This leads to a simplified case where the bladder of the bags that encase the water is neglected, despite the benefits the bladder would bring. Table 7.3 shows the drastic improvement that polyethylene provides to the shield. An increase in production of $7.6 \%$ for dose equivalent is comparable to the increase it would provide to the effective dose equivalent, with slight variance due to the weighting factors. 


\section{Table 7.3: Dose Equivalent Comparison Including Bladder}

\begin{tabular}{lcc} 
Test Case & Dose Equivalent, mSv & $\%$ Variance \\
\hline Simplified Case & 581.4 & - \\
Case with Polyethylene & 537.1 & 7.6 \\
\hline
\end{tabular}

\subsubsection{Carrington Event}

Table 7.4 shows the radiation exposure of an astronaut within the Orion capsule during the Carrington Event. This is a massive event that leads to a heavy dosage in a short amount of time. The permanent water shield clearly outperforms the current Orion plan, although both scenarios lead to dangerous levels of exposure. The lifetime limit for a 25-year old male is $700 \mathrm{mSv}$, and a 55-year old male is $3000 \mathrm{mSv}$. The lifetime limit for a 25 -year old female is $400 \mathrm{mSv}$, and a 55 -year old female is $1700 \mathrm{mSv}$. Should astronauts be exposed to a massive event on this order of magnitude, they would approach lifetime radiation limits unacceptably quick. A 25-year old female would exceed $70 \%$ of her lifetime limit in the span of this one SPE. On a longduration mission, this would ensure the astronaut exceeds the NASA standard 3\% REID. Even a 55-year old male would exceed $10 \%$ of lifetime radiation limit from the event. A storm of this magnitude would be crippling to the crew and quite possible life-threatening.

The dosage Table 7.5 gives the short-term dosage effects on blood forming organs. This is a good indication for NASA to project against the astronaut's risk of acute radiation syndrome. Described earlier, NASA has a set 30-day limit of astronauts recieving $250 \mathrm{mSv}$ of dosage to Blood Forming Organs. Had astronauts been in the path of this event, it would have been catastrophic. The event only spanned several days, and even with an emergency bunker of water, astronauts would have exceeded the limit. Pair this event with 30-days of exposure to GCR and the astronauts in 
transit would have most likely suffered from an intense form of radiation sickness, and quite possibly in-flight death. The permanent water shield reduced the effects of the event by $5.2 \%$, however the astronauts are still exposed to a heavy radiation dose.

\section{Table 7.4: Carrington Event Radiation Exposure: Long-Term Effects}

\begin{tabular}{lcc} 
Parameter & Permanent Shielding & Emergency Bunker \\
\hline CAM Effective Dose Equivalent, mSv & 277.5 & $292.6 \pm 2.6$ \\
CAF Effective Dose Equivalent, mSv & 278.7 & $293.3 \pm 2.6$ \\
\hline
\end{tabular}

Table 7.5: Carrington Event Radiation Exposure: Short-Term Effects

\begin{tabular}{lcc}
\hline Parameter & Permanent Shielding & Emergency Bunker \\
\hline CAM BFO Dose Equivalent, mSv & 268.0 & $282.4 \pm 2.5$ \\
CAF BFO Dose Equivalent, mSv & 278.4 & $294.1 \pm 2.6$ \\
\hline
\end{tabular}

There is an improvement from the permanent water shield, although it does not have as large of an effect as hoped to lower dosages. The thin coverage the on-board water provides is not ideal and could produce much more successful results with an increase in water mass. This will be investigated for each scenario to provide more data to support the case.

In addition, the shielding configuration creates a considerable change in the radiation dosage within the vehicle. All tests assumed the exterior shield would consist of aluminum lithium and the water would lie on the inside. This is beneficial, as it allows for the maximum thickness of water in the shielding configuration. When the water is in the interior of the shield it is $0.8 \mathrm{~cm}$ thick around the entire vehicle. If the water was relocated to the exterior, it would result in a loss of thickness. This is due to the increased surface area the pre-allocated $240 \mathrm{~kg}$ of water would have to cover. Water placed on the exterior would limit the thickness to $0.58 \mathrm{~cm}$. Figure 7.2 shows 
the effects of changing the shielding configuration to protect against the SPE environment. Interestingly, water is considerably more beneficial on the exterior portion of the shielding for SPE. Figure 7.2 shows the resulting effective dose on a phantom body depending on the location of water within the shield. The exterior part of the shield is the left side of the graph (thickness of $0 \mathrm{~g} / \mathrm{cm}^{2}$ ). The right side is the interior of the spacecraft (thickness of $45.33 \mathrm{~g} / \mathrm{cm}^{2}$ ). Figure 7.2 lines up with Figure 7.1 spatially showing the dosage drop as water is moved to the exterior of the radiation shield. This trend will be discussed in further detail later in this section.

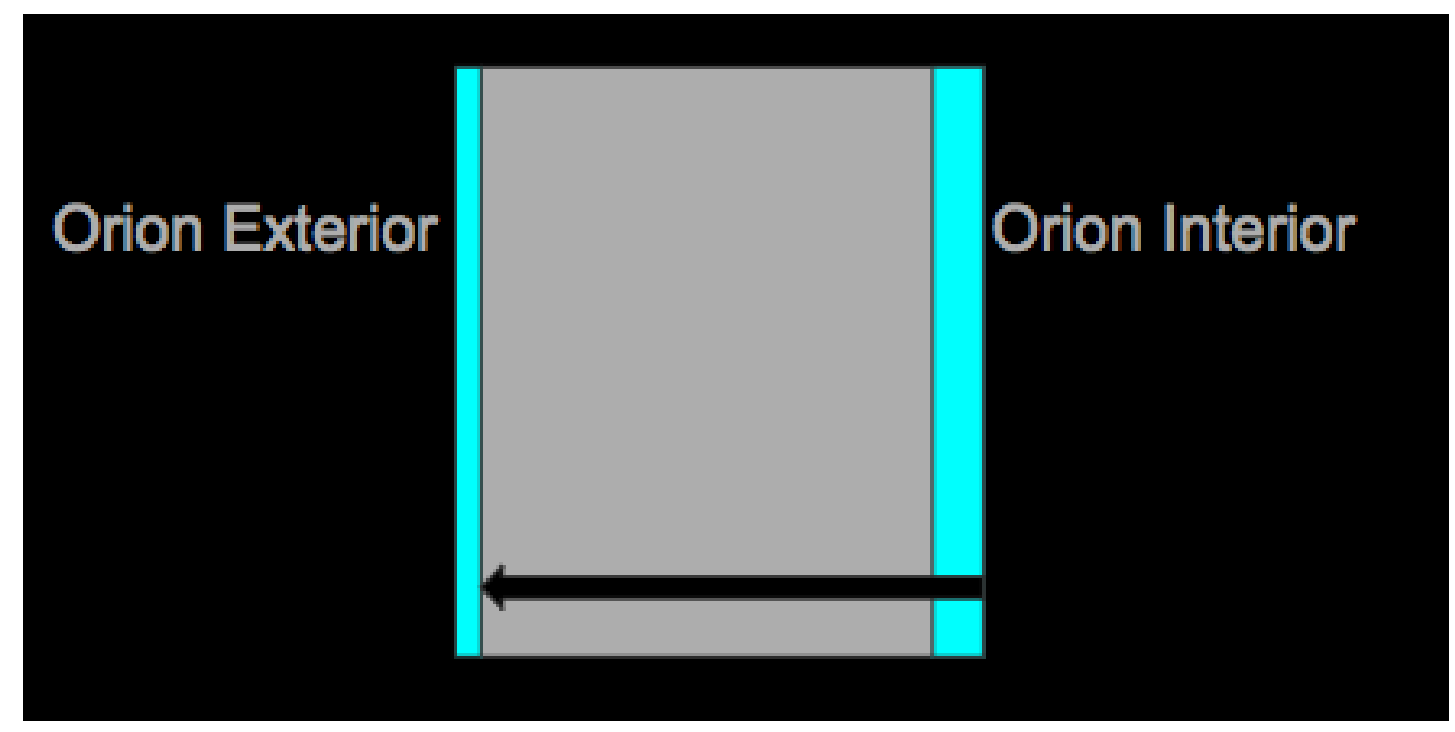

Figure 7.1: Orion Shield Cutout with Water Configuration

Table 7.6 and Table 7.7 provide the results for radiation shielding if the water was placed on the exterior of the radiation shield for effective dose equivalent and average dose equivalent for Blood Forming Organs respectively.

Table 7.6: Radiation Exposure with Water on Exterior: Long-Term Effects

\begin{tabular}{lcc} 
Parameter & Permanent Shielding & Emergency Bunker \\
\hline CAM Effective Dose Equivalent, mSv & 241.4 & $294.3 \pm 2.6$ \\
CAF Effective Dose Equivalent, mSv & 243.1 & $293.3 \pm 2.6$ \\
\hline
\end{tabular}




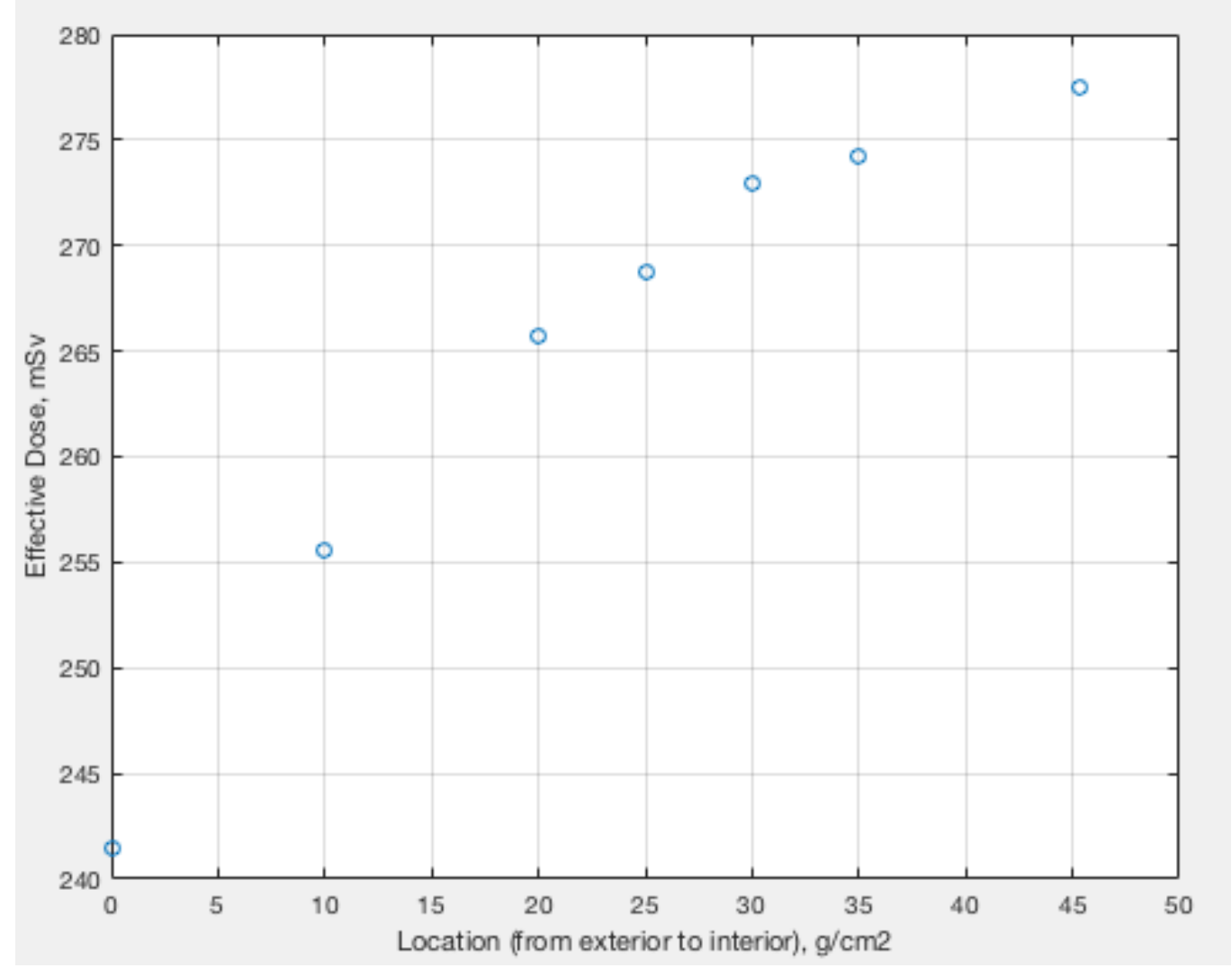

Figure 7.2: Water Shield Configuration within the SPE Environment

This configuration of the permanent water shield reduced the effects of the event by $17.5 \%$ compared to the current NASA plan using an emergency bunker. This puts astronauts in a much safer state than the other shield configuration as well as the current emergency bunker plan. This is a substantial improvement from the other configuration and needs to be considered.

Table 7.7: Radiation Exposure with Water on Exterior: Short-Term Effects

Parameter Permanent Shielding Emergency Bunker

$\begin{array}{lll}\text { CAM BFO Dose Equivalent, } \mathrm{mSv} & 234.5 & 282.4 \pm 2.5\end{array}$

$\begin{array}{lll}\text { CAF BFO Dose Equivalent, } \mathrm{mSv} & 242.7 & 294.1 \pm 2.6\end{array}$

Again, the $\mathrm{BFO}$ dose is considerably lower with the water on the exterior of the shield. Despite the success from the permanent water fixture, there would still be 
complications for some astronauts depending on age and gender. A 25-year old female astronaut would be exposed to $60.8 \%$ of her lifetime radiation limit in the span of one solar particle event in this case, as opposed to the $70 \%$ she would have been exposed to with water on the interior. If this occurred at the beginning of a Mars or moon mission the astronaut would certainly exceed the radiation limit. In comparison, a 55-year old female astronaut would be exposed to $14.3 \%$ of her lifetime radiation limit. A 25-year old male astronaut would be exposed to $34.5 \%$ of his lifetime radiation limit in the span of one solar particle event. Like the case for the female, it is likely the astronaut would exceed the radiation limit if this occurred in a long-duration mission. In comparison, a 55-year old male astronaut would be exposed to $8.0 \%$ of his lifetime radiation limit.

This is a major event that is extremely rare in magnitude. In a worst-case scenario, it would be ideal to have older astronauts in transit to reduce the long-term effects of the radiation dosages. Including the water in the shield does reduce the risk of astronauts developing acute radiation considerably.

The new shielding configuration is considerably more effective in radiation prevention, but is infeasible for spacecraft design. Water should not be placed on the exterior of the vehicle due to the possibility of a micro-meteoroid strike. Any penetration on the exterior of the shield could result in loss of water, which is an unacceptable failure for crewed missions. A solution to this will be proposed following the analysis of the GCR environment.

As expected, increasing the thickness of the water shield lowers the dose of radiation within the vehicle. The trend is shown in Figure 7.3 with the dosage reducing considerably with an increase of water for the mission. In this case, the water is in the interior portion of the shield. The trend is the same if calculated with the water on the exterior portion of the spacecraft. 


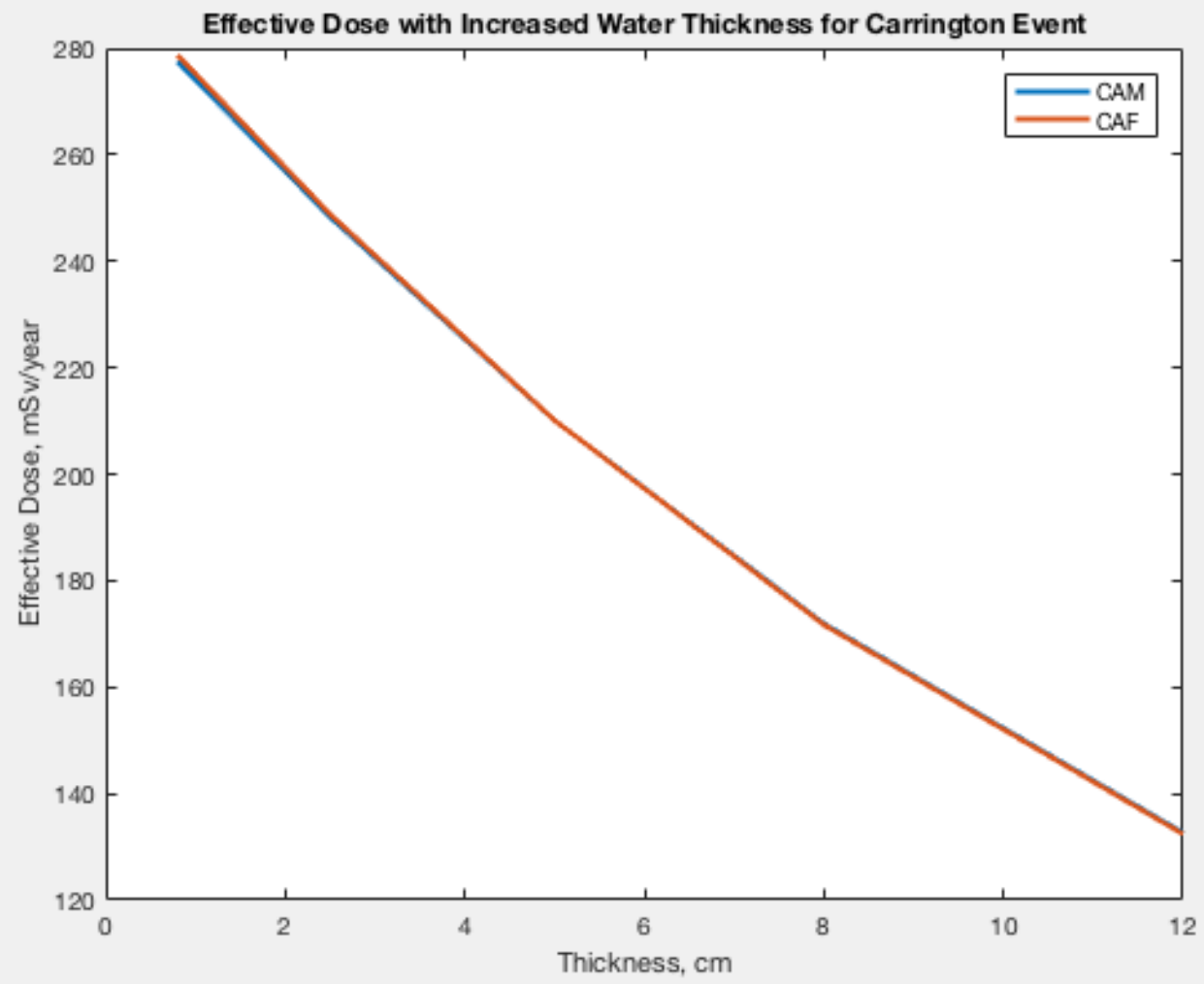

Figure 7.3: Radiation Dosage Vs Shielding Thickness for Carrington Event

Table 7.8 shows how the increased water impacts other aspects of the mission. As described earlier, the Orion program is intended to recycle water to minimize the required mass, however it would be beneficial to radiation shielding to bring a surplus. If there is no water recycling utilized on the transit for a Mars mission, an approximately $10 \mathrm{~cm}$ thickness shield of water could be utilized to protect the astronauts in transit. The effectiveness (\%) in Table 7.8 shows compares the effect of each water shield with the baseline emergency bunker for the Carrington event specifically. If possible, a water shield thickness of $10-12 \mathrm{~cm}$ would prove immensely helpful in defending against radiation. 
Table 7.8: Results of Increasing Shield Thickness

\begin{tabular}{lccc}
\hline $\begin{array}{l}\text { Water Shield Thickness } \\
(\mathrm{cm})\end{array}$ & $\begin{array}{c}\text { Weight of Water } \\
(\mathrm{kg})\end{array}$ & $\begin{array}{c}\text { Mission Duration } \\
(\text { days })\end{array}$ & $\begin{array}{c}\text { Effectiveness } \\
(\%)\end{array}$ \\
\hline 0.8 & 240 & 28.9 & 5.2 \\
1 & 325.8 & 36.2 & 15.14 \\
2 & 647.5 & 71.9 & 18.39 \\
4 & 1279 & 142.1 & 25.98 \\
6 & 1895 & 210.6 & 32.15 \\
8 & 2495 & 277.2 & 41.25 \\
10 & 3079 & 342.1 & 48.62 \\
12 & 3649 & 405.4 & 54.68 \\
\hline
\end{tabular}

\subsubsection{October 1989}

The event in October of 1989 was a massive solar storm directed towards Earth. It is of much smaller magnitude than the Carrington event. Table 7.11 shows the longterm effects of the event through the use of effective dose equivalent in the OLTARIS phantom bodies. Table 7.12 shows the short-term effects of the event through the use of average dose equivalent from blood forming organs. For all of these scenarios, the water is on the interior portion of the shield.

Table 7.9: October 1989 Event Radiation Exposure: Long-Term Effects Parameter Permanent Shielding Emergency Bunker

\begin{tabular}{lll}
\hline CAM Effective Dose Equivalent, mSv & 89.77 & $96.95 \pm 0.85$ \\
CAF Effective Dose Equivalent, mSv & 90.14 & $97.30 \pm 0.85$ \\
\hline
\end{tabular}

The permanent water shield provides better shielding than the current emergency bunker plan. In addition, the difference is greater than in the Carrington event. The permanent shield protects against total effective dose $7.6 \%$ better than the current 
Table 7.10: October 1989 Event Radiation Exposure: Short-Term Effects

Parameter Permanent Shielding Emergency Bunker

$\begin{array}{lll}\text { CAM BFO Dose Equivalent, } \mathrm{mSv} & 86.98 & 94.17 \pm 0.83\end{array}$

$\begin{array}{lll}\text { CAF BFO Dose Equivalent, } \mathrm{mSv} & 90.03 & 97.92 \pm 0.86\end{array}$

plan. This provides a trend of increased performance for decreased solar event size magnitude. This is encouraging as a vast majority of SPEs are smaller magnitude.

Again, the effect of the radiation shielding differs based on the location of the water in the shields. The following tables demonstrate the effect of radiation when the water is located on the furthermost portion of the shield from the astronauts. The results produce a similar trend as that shown for the Carrington event in Figure 7.2.

Table 7.11: Radiation Exposure with Water on Exterior: Long-Term Effects

\begin{tabular}{lcc} 
Parameter & Permanent Shielding & Emergency Bunker \\
\hline CAM Effective Dose Equivalent, mSv & 86.07 & $96.44 \pm 0.85$ \\
CAF Effective Dose Equivalent, mSv & 86.55 & $96.80 \pm 0.85$ \\
\hline
\end{tabular}

Table 7.12: Radiation Exposure with Water on Exterior: Short-Term Effects

\begin{tabular}{lcc} 
Parameter & Permanent Shielding & Emergency Bunker \\
\hline CAM BFO Dose Equivalent, mSv & 83.66 & $93.65 \pm 0.83$ \\
CAF BFO Dose Equivalent, mSv & 86.45 & $97.20 \pm 0.86$ \\
\hline
\end{tabular}

In this scenario, a 25-year old female astronaut would be exposed to $21.6 \%$ of her lifetime radiation limit. In comparison, a 55 year old female astronaut would be exposed to $5.1 \%$ of her lifetime radiation limit. A 25-year old male astronaut would be exposed to $11.9 \%$ of his lifetime radiation limit. In comparison, a 55 year old male astronaut would be exposed to $2.8 \%$ of his lifetime radiation limit. These numbers are much more acceptable than those calculated from the Carrington event, although 
NASA would still prefer older astronauts to younger. The emergency bunker results do not pose an immediate threat to lifetime or short-term limits, however, the results have not been paired with the effects of GCR.

The trends indicate that the protection provided by the water shielding increases as the magnitude in the event increases. The permanent water shield always protects at a better rate against solar particle events than an emergency bunker, and increases in efficiency for smaller storms. This could be critical for future missions to protect against the SPE environment.

Similar to the Carrington case, the radiation dosage was determined for a variable thickness of water shielding. Again, in this case, the water is located in the interior portion of the shield. This is displayed in Figure 7.4, and continues the expected trend of reduced dosage. This shows a reduced amount of water recycling could reduce the effective dose by a factor of two, which would substantially lower the risks for astronauts in deep-space missions.

For both Solar Particle Events considered in this study, it is considerably better to consider increasing the amount of water included on-board for radiation shielding. There are other beneficial effects that an increased amount of water could provide to a deep space mission. One huge asset could be to have redundant water in the scenario of a failure in the water recycling system. The water could possibly be utilized in other methods such as thermal to increase the merit for raising the mass budget for the material. In addition, it is possible to consider alternative options, such as docking with the ISS or another spacecraft in-flight to increase the amount of on-board water without compromising the mass of the vehicle at launch.

Water is clearly beneficial, even in small amounts, to the protection of astronauts against solar events in deep space. As shown in both of the events discussed, it would be considerably beneficial to move the water layer closer to the exterior portion of 


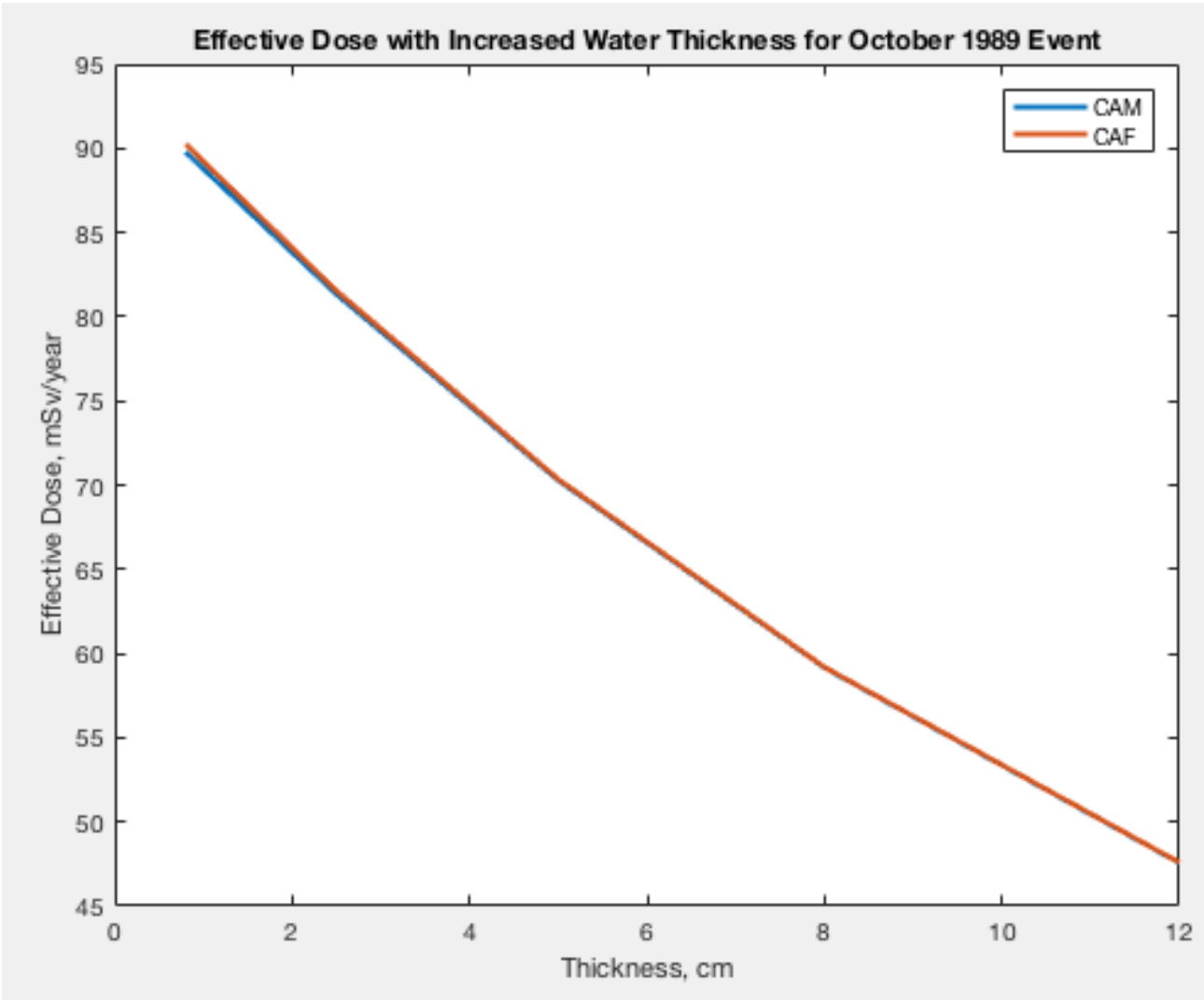

Figure 7.4: Radiation Dosage Vs Shielding Thickness for the October 1989 Event

the shield as well as increase the amount of water brought on the mission.

\subsection{GCR Analysis}

As described in previous sections, the GCR environment poses a particularly difficult problem for radiation shielding due to the high ionization power of HZE particles. It has been shown in previous studies that water has the efficacy to contribute to shielding, however this case calls for a thin shield. Table 7.13 shows the minor effect water contributes over the course of a 21-day mission. Although it does contribute, the effects are minimal and hardly inspire change. The permanent water shield provides better coverage by approximately $1 \%$. This is not surprising due to the nature of the GCR environment, and there are several more parameters to introduce into the tests 
to yield more favorable results for a permanent water shield.

Table 7.13: GCR

\begin{tabular}{lcc}
\hline Parameter & Permanent Shielding & Baseline Orion \\
\hline CAM Effective Dose Equivalent: & & \\
Total Mission Dose, mSv & 17.76 & 17.94 \\
\hline CAM Effective Dose Equivalent & & \\
Rate, mSv/year & 308.8 & 311.8 \\
\hline CAF Effective Dose Equivalent: & & \\
Total Mission Dose, mSv & 17.91 & 17.97 \\
\hline CAF Effective Dose Equivalent & & 312.4 \\
Rate, mSv/year & 311.4 & \\
\hline
\end{tabular}

Although the water doesn't provide substantial assistance, the magnitude of dosage seen on a 21-day Moon mission is relatively low. This is a large reason why it is the current plan for the capsule, as it is well within reach of radiation guidelines. For these shorter missions, the largest concern for astronauts becomes SPE radiation.

For all tests, it was assumed the water lined the inner side of the spacecraft shield. This was to maximize the thickness of water used to shield the astronauts. Further tests analyze the effects of moving the water from the inner to the outer portion of the vehicle shield, as well as increasing the volume of water launched with the astronauts.

Figure 7.5 shows that the water location within the shield leads to increased performance. The left side of the figure depicts the outer-most portion of the shield and right-side represents the interior. This differs with an inverse relationship to the SPE results. Whereas SPE results indicates water should be on the exterior, the GCR environment was protected better with the water placed on the inside of the shield. This is more practical as water should not be on the exterior of the spacecraft shielding. This will be discussed in more detail in the following subsection. 


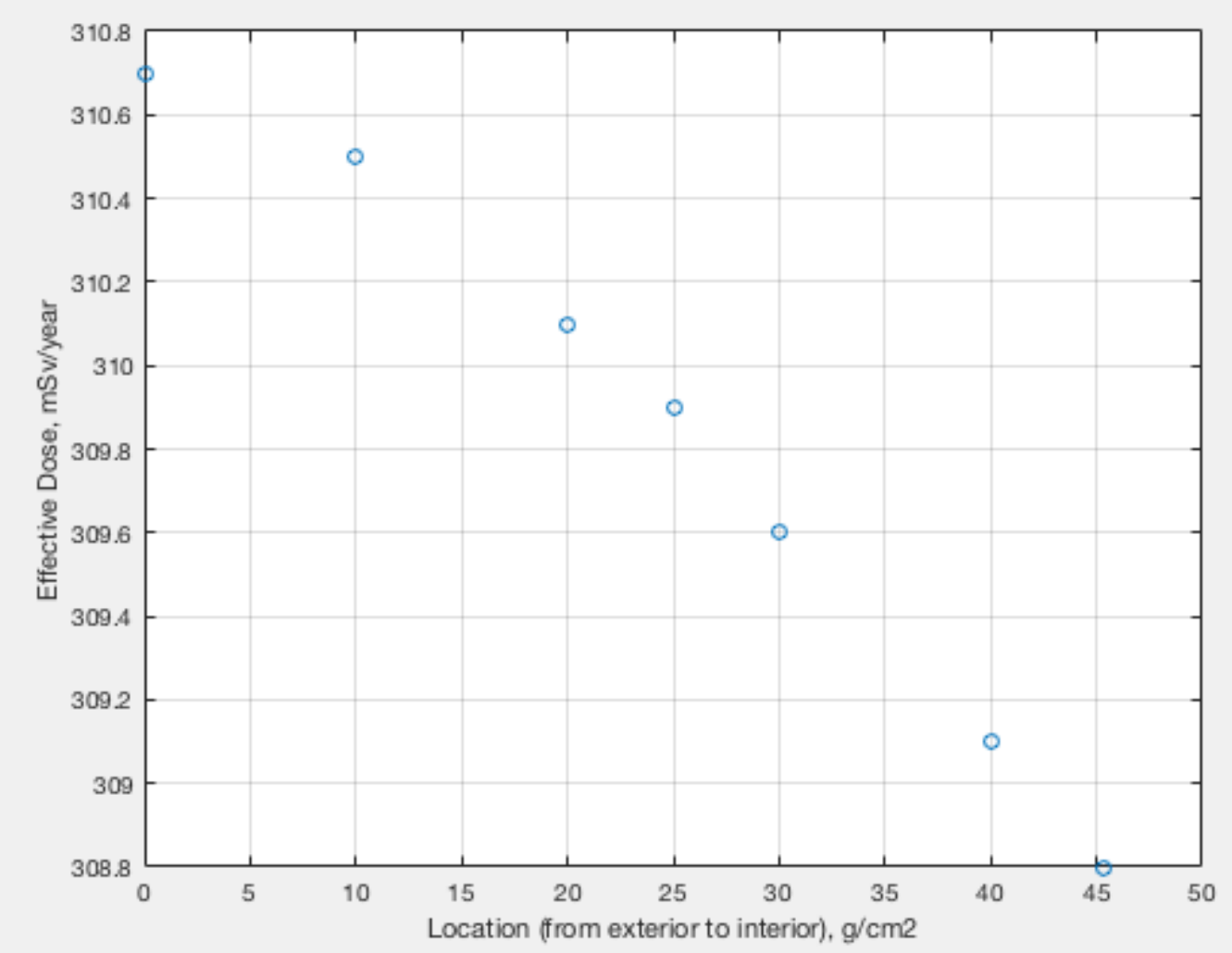

\section{Figure 7.5: Water Shield Configuration within GCR environemnt}

Figure 7.6 provides the data for the effect of the increased thickness of water on the radiation dosage. The trend follows that of the SPEs as it decreases dramatically with the increase in water mass.

If the amount of water was increased to $3079 \mathrm{~kg}$ for a year-long mission, the shield would produce nearly $12 \%$ more coverage over the course of the mission. This is a considerable bump from the $1 \%$ documented previously. In terms of a Mars mission the increased water could be the difference between astronauts meeting the 3\% REID requirement or exceeding it. 


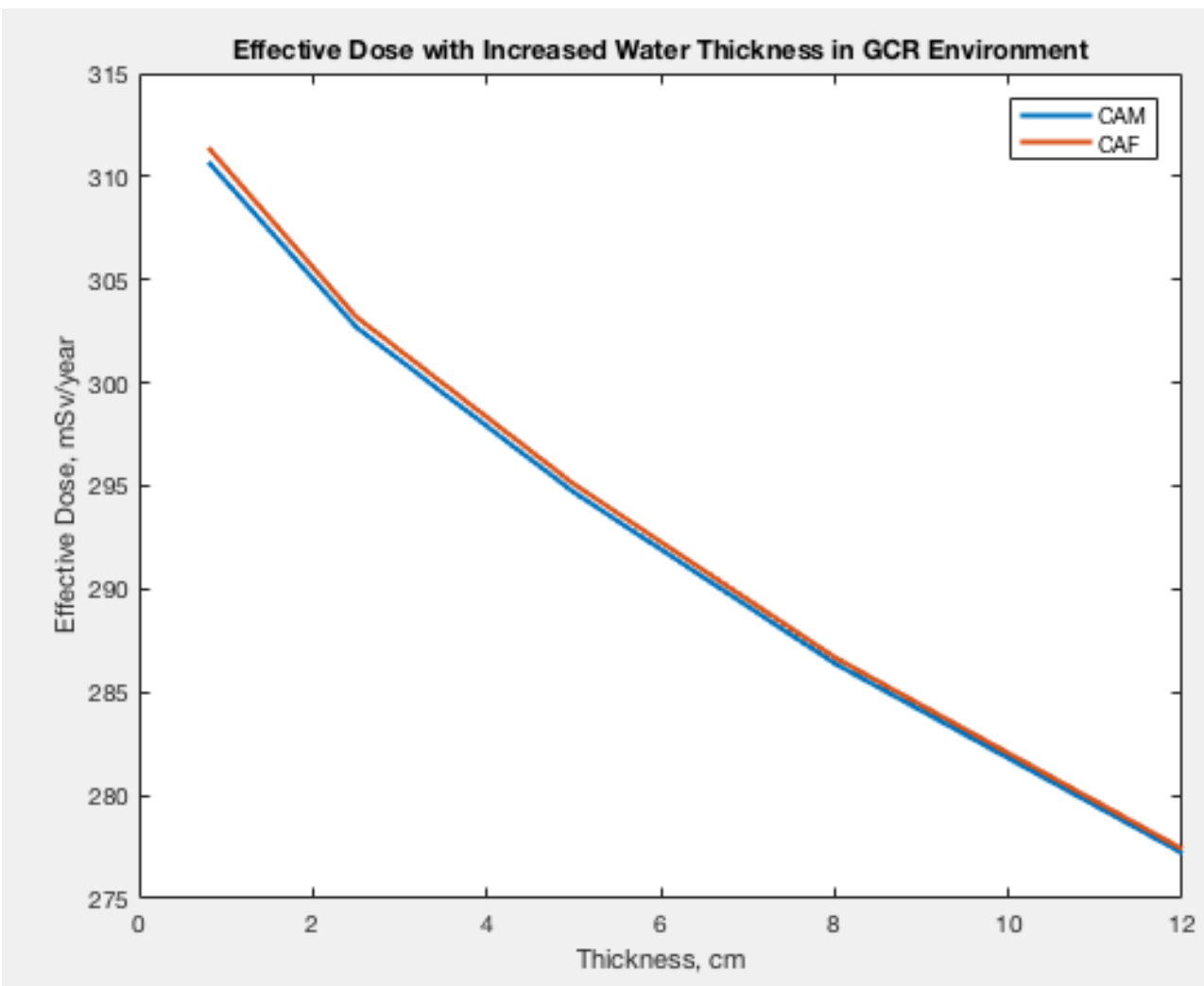

Figure 7.6: Radiation Dosage Vs Shielding Thickness in the GCR Environment

\subsection{Shield Configuration}

The trend line for GCR is nearly opposite of that for solar particle events. One possible reason for the trend is secondary radiation from the GCR particles colliding with the aluminum shielding. Water is known to be a good material for reducing the energy of particles that traverse through it. It is possible the water retards a fraction of the aluminum fragments making it a better investment to be placed at the inner portion of the shielding. On the interior the water can absorb as much secondary radiation as possible. In the scenario where water is on the exterior, the thin thickness of the shield prevents the water from slowing down any GCR or secondary radiation. The HZE particles can't be slowed down by such a thin thickness of water to reduce the effect of the GCR environment any further. As the water shield increases in 
thickness, it becomes more possible to consider the effect of water on HZE particles.

The water does a far better job slowing down the solar particles, which are generally less energetic than in the GCR environment. This is expected due to the minimal thickness of the shield used for the analysis. Coming into the research project, it was desired to utilize water as a permanent shielding fixture to combat GCR constantly. This did not come to full fruition until additional water was added to the system to improve the effectiveness of the shield against HZE particles. It was not expected that the permanent water shield would outperform the emergency bunker at such an indisputable margin. For this reason, the water is better placed as the front line of shielding for SPEs.

As mentioned earlier, the water should not be an exterior portion of the spacecraft. This creates an issue as it serves much more utility in the front line of radiation defense. With the limitation from micro-meteoroids and the data showing the inverse relationship with water in the GCR environment, it is best to locate the water closer to the middle of the aluminum lithium shielding. As the water shielding increases in thickness, it decreases the sensitivity to location within the configuration.

\subsection{Shield Thickness}

In addition to changing the shielding configuration, increasing the volume of water will increase shielding efficiency. As expected, the increased volume of water creates a higher performing shield. This decrease in radiation exposure comes with a cost in mission planning due to the increase in mass.

With the current numbers, shorter duration Moon missions are well within reach, barring a massive solar event on the scale close to the Carrington event. With the numbers produced in this study, a large SPE on the order of the 1989 October event would not exceed the 3\% REID. Crewed Mars missions create a much larger issue as 
the mission travel time consumes over a year, without considering the time spent on the planet. With the current iteration of Orion spacecraft, 12-months of deep space transit has a considerable probability of risk to astronauts. This does not account for the radiation exposure certain to occur on the surface of the planet, as well as any previous radiation exposure should an astronaut not be flying their first mission. This poses a huge risk for NASA, especially if a large solar event occurs in-flight. Through both cases of SPEs and the GCR environment, it is apparent the increase in water mass is crucial to reducing radiation dosages. This could allow for longer duration missions that are currently unachievable under NASAs radiation guidelines. 
Chapter 8

CONCLUSION

Utilizing water as a permanent fixture in radiation shielding is shown to be a beneficial strategy. It provides critical protection against devastating solar storms and decreases the reliance of warning systems to create emergency storm bunkers. As shown in the testing section, water provides considerable protection against radiation, especially from the sun. The permanent shield provides up to $17.5 \%$ better coverage than the current emergency protection plan under exposure from the Carrington Event. Due to this vital attribute, water shielding should be utilized for human spaceflight missions. Minimizing the amount of water required for the astronauts decreases the mass burden for launch, but could be costly for radiation protection. It could prove to be more optimal to bring an excess of water for longer duration missions despite the efficiency of water recycling systems in order to bolster the effects of shielding.

In the event of a massive solar storm, a permanent water shield could very well be the difference between life and death for the astronauts on board and should be considered as an option. The results found that a water shield of just $0.8 \mathrm{~cm}$ would lower the dosage on BFO by over $17 \%$ to $234.5 \mathrm{mSv}$. This brings the short-term dose from the event below the NASA limit of $250 \mathrm{mSv}$ over a 30-day span. Often spacecraft engineering relies on flight heritage for proven success. This limits the desire to create new systems such as putting a critical resource in spacecraft shielding, however, it should not be ignored as an option.

Due to strict lifetime radiation limits, it is much more beneficial to launch older astronauts. This could lead to complications if those astronauts have flown before, leading to a necessity for a very specific target astronaut pool. Due to the uncertainty of the radiation environment and age-based radiation accumulation restrictions, the 
use of younger astronauts poses a much greater health risk. For a Mars mission, it is a near-necessity to have older astronauts embark on their first mission.

There are many assumptions that had to be made throughout the process of determining the test vehicle and test scenarios. These assumptions and simplifications lead to radiation results that should not be compared to the Orion vehicle directly, but rather, should be used as a means to determine the effectiveness of permanent water shielding in place of the current method of creating an emergency water bunker. This thesis proposes and shows that water should be incorporated as a full time shield to best protect the astronauts in transit. The emergency radiation bunker can still be utilized to protect the astronauts with food and storage, but water should be a permanent fixture for future crewed missions.

\subsection{Future Work}

There is a known trend that water is beneficial to shielding spacecraft for deep space radiation. This paper proves that utilizing water as a permanent fixture is more beneficial than current NASA plans to create an emergency bunker. Moving forward, it would be useful to obtain more data from NASA to be able to verify dosage levels for OLTARIS. There were several assumptions made through the course of this project, some of which are due to limitations in current radiation models; others are due to a lack of free information. With the ability to verify dosage calculations, the results could rely less on trends and more on specific dosage levels and lifetime limits for long duration missions.

It would also provide useful to delve deeper into the shielding configuration trends brought up in this study. There appears to be a clear indication that there is a "sweet spot" when placing the water to shield a vehicle. A study on the best location to

place the water within the shield depends heavily on Micro-Meteoroid and Orbital 
Debris (MMOD) restrictions that are not addressed in this study. It would be useful to determine how close to the exterior is acceptable to place water without posing too high a risk to the astronauts and the water supply.

Further work could include a statistical analysis in the probability of being impacted by solar events per mission. This could allow for a more in-depth analysis of radiation dosage seen per mission and narrow down the dosage seen compared to an astronaut's career limit. This would prove more useful when the dosage levels are verified with in-flight radiation results from NASA.

If the permanent water shield was determined to be worth the investment to develop, there are several engineering hurdles to designing the system. One major hurdle is surviving the launch vibration and acoustic environment with crucial potable water for the astronauts. One simple solution is to hold the water in tanks for launch and then pump into the shield, but this requires analysis and trade studies to determine the worth of the design. In addition, it is important to design the system to function in micro-gravity, possibly with capillary action. In addition, it is crucial to integrate the recycling of water into the system for long duration missions. All of these steps provide a path forward for a design to be accepted for future space travel, and for future astronauts to have lower risk missions. 


\section{BIBLIOGRAPHY}

[1] "Intersections of Rays and Triangles".

"http://geomalgorithms.com/a06-_intersect-2.html", 2010. Web. Accessed: 4-30-2018.

[2] "Everyday Calculations: Conical Frustum".

"http://everydaycalculation.com/volume-calculator/conical-frustum", 2012. Accessed: 2-30-2018.

[3] J. C. Chancellor. "Space Radiation: The Number One Risk to Astronaut Health Beyond Low Earth Orbit". Life, 4:491 - 510, 2014.

[4] S. Chiavassa. "Dosimetric comparison of Monte Carlo codes (EGS4, MCNP, MCNPX) considering external and internal exposures of the zubal phantom to electron and photon sources". Radiation Protection Dosimetry, 116, 2005.

[5] F. A. Cucinotta. "Space Radiation Cancer Risk Projections and Uncertainties". NASA Scientific and Technical Information.

[6] F. A. Cucinotta. "Radiation Risk Acceptability and Limitations". NASA Technical Paper, 2010.

[7] M. Garcia. "Scientists and Engineers Evaluate Orion Radiation Protection Plan". "https://www.nasa.gov/feature/scientists-and-engineers-evaluate-orionradiation-protection-plan", 2016.

[8] B. Giuseppe. "The FLUKA Code: An Accurate Simulation Tool for Particle Therapy". Frontiers in Oncology, 6, 2016. 
[9] M. Gleber. "The Difference Between Flares and CMEs. NASA Technical Paper, 2014.

[10] M. Gonzalez-Dorbecker. "DEVELOPMENT OF TOOLS NEEDED FOR RADIATION ANALYSIS OF A CUBESAT DEPLOYER USING OLTARIS". California Polytechnic State University San Luis Obispo, 2015.

[11] D. Hathaway. "The Solar Cycle". NASA Ames Research Center.

[12] J. Heinbockel. "Comparison of Transport Codes, HZETRN, HETC and FLUKA, Using 1977 GCR Solar Minimum Spectra". NASA Technical Paper, 2009.

[13] K. Henry. "Student Project to Protect Astronauts".

"https://www.nasa.gov/centers/langley/news/researchernews/rn_studentproject.html", 2010.

[14] D. Liskowsky. "Human Integration Design Handbook". NASA Handbook, 2014.

[15] E. Marsch. "Kinetic Physics of the Solar Corona and Solar Wind ". Solar Physics, 2006.

[16] NASA. "Human Needs: Sustaining Life During Exploration".

"https://www.nasa.gov/vision/earth/everydaylife/jamestown-needs-fs.html", 2007.

[17] NASA. "Model of 1859 Carrington Event".

"https://www.nasa.gov/content/goddard/model-of-1859-carrington-event/", 2014 .

[18] NASA. "Orion Quick Facts". Brochure, 2014.

[19] P. M. ONeill. "Badhwar-ONeill 2014 Galactic Cosmic Ray Flux Model Description ". NASA Technical Paper, 2015. 
[20] E. Parker. "The Passage of Energetic Charged Particles Through Interplanetary Space". Planetary and Space Science, 13(1):9 - 49, 1965.

[21] T. Phillips. "Sickening Solar Flares. NASA Technical Paper, 2005.

[22] J. Rask. "Radiation Educator Guide". The Radiation Challenge, 2008.

[23] C. Rodger. "Atmospheric impact of the Carrington event solar protons ". Geophysical Research, 2008.

[24] W. Schimmerling. "The Space Radiation Environment: An Introduction". NASA Technical Paper, 2011.

[25] E. Semones. "Radiation Health Risk Protections". "https://www.nasa.gov/sites/default/files/files/8_NAC_HEO_SMD_Committee_Semones_Apri 2015.

[26] V. I. Shematovich. "Monte Carlo model of electron transport for the calculation of Mars dayglow emissions". Advancing Earth and Space Science, 2008.

[27] L. Simonsen. "Radiation Protections for Human Missions to the Moon and Mars". NASA Technical Paper, 1991.

[28] R. C. Singleterry. "On-Line Tool for the Assessment of Radiation in Space. NASA Technical Paper, 2010.

[29] T. Slaba. "Space Radiation Environment". NASA Powerpoint Presentation, 2015.

[30] L. W. Townsend. "Critical Analysis of Active Shielding Methods for Space Radiation Protection". The University of Tennessee, 2004.

[31] J. Wilson. "Verification and Validation: High Charge and Energy (HZE) Transport Codes and Future Development". NASA Technical Paper, 2005. 